\title{
Corner contributions to holographic entanglement entropy in $\mathrm{AdS}_{4} / \mathrm{BCFT}_{3}$
}

\author{
Domenico Seminara, ${ }^{a}$ Jacopo Sisti $^{b}$ and Erik Tonni ${ }^{b}$ \\ ${ }^{a}$ Dipartimento di Fisica, Università di Firenze and INFN Sezione di Firenze, \\ Via G. Sansone 1, 50019 Sesto Fiorentino, Italy \\ ${ }^{b}$ SISSA and INFN, \\ via Bonomea 265, 34136 Trieste, Italy \\ E-mail: seminara@fi.infn.it, jsisti@sissa.it, erik.tonni@sissa.it
}

ABSTRACT: We study the holographic entanglement entropy of spatial regions with corners in the $\mathrm{AdS}_{4} / \mathrm{BCFT}_{3}$ correspondence by considering three dimensional boundary conformal field theories whose boundary is a timelike plane. We compute analytically the corner function corresponding to an infinite wedge having one edge on the boundary. A relation between this corner function and the holographic one point function of the stress tensor is observed. An analytic expression for the corner function of an infinite wedge having only its tip on the boundary is also provided. This formula requires to find the global minimum among two extrema of the area functional. The corresponding critical configurations of corners are studied. The results have been checked against a numerical analysis performed by computing the area of the minimal surfaces anchored to some finite domains containing corners.

KEYwords: AdS-CFT Correspondence, Boundary Quantum Field Theory

ARXIV EPRINT: 1708.05080 


\section{Contents}

1 Introduction 2

$\begin{array}{lll}2 & \text { Constraining the corner functions } & 7\end{array}$

3 Warming up: corner functions in $\mathrm{AdS}_{4} / \mathrm{CFT}_{3} \quad 10$

$\begin{array}{lll}3.1 & \text { Single drop } & 11\end{array}$

$\begin{array}{ll}3.2 & \text { Two drops with the same tip } \\ \end{array}$

4 Holographic entanglement entropy in $\mathrm{AdS}_{4} / \mathrm{BCFT}_{3} \quad 17$

5 The half disk and the infinite strip $\quad 19$

$\begin{array}{ll}5.1 \text { Half disk centered on the boundary } & 19\end{array}$

5.2 Infinite strip adjacent to the boundary 20

$\begin{array}{lll}5.3 & \text { Infinite strip parallel to the boundary } & 24\end{array}$

6 Infinite wedge adjacent to the boundary $\quad 26$

6.1 Limiting regimes of the corner function 31

6.2 Relations with the stress tensor 33

$\begin{array}{lll}7 & \text { Infinite wedge with only the tip on the boundary } & 35\end{array}$

8 Conclusions $\quad 40$

A On the numerical analysis $\quad 42$

B On the half disk centered on the boundary 45

C Infinite strip adjacent to the boundary in generic dimension 46

D Infinite strip parallel to the boundary in generic dimension 53

E On a modification for the holographic entanglement entropy 56

F On the infinite wedge adjacent to the boundary $\quad 59$

F.1 Minimal surface condition $\quad 61$

F.2 Intersection between the minimal surface and the brane 62

F.3 Area of the minimal surface 64

F.4 On the limiting regimes of the corner function 66

$\begin{array}{lll}\text { F.4.1 Large } q_{0} \text { regime } & 68\end{array}$

F.4.2 Small $q_{0}$ regime $\quad 69$

F.5 A relation between the infinite wedge and the infinite strip $\quad 70$

$\begin{array}{ll}\text { G The coefficient } A_{T} \text { from holography } & 73\end{array}$ 


\section{Introduction}

Entanglement entropy has been largely studied during the last two decades in quantum field theory, quantum gravity, quantum many-body systems and quantum information (see [1-4] for reviews). Nowadays it is a powerful quantity to understand some properties of quantum systems.

Given a quantum system whose Hilbert space $\mathcal{H}=\mathcal{H}_{A} \otimes \mathcal{H}_{B}$ is bipartite, by considering the density matrix $\rho$ characterising the state of the system, one can introduce the $A$ 's reduced density matrix $\rho_{A}=\operatorname{Tr}_{\mathcal{H}_{B}} \rho$. We study only bipartitions associate to spatial subsystems. The entanglement entropy between $A$ and $B$ is defined as the Von Neumann entropy of the reduced density matrix $\rho_{A}$, namely

$$
S_{A}=-\operatorname{Tr}\left(\rho_{A} \log \rho_{A}\right)
$$

In the same way, one can construct the $B$ 's reduced density matrix $\rho_{B}=\operatorname{Tr}_{\mathcal{H}_{A}} \rho$ and the corresponding entanglement entropy $S_{B}$. When the state $\rho$ is pure, we have $S_{A}=S_{B}$.

In this manuscript we consider the entanglement entropy in certain conformal field theories (CFTs) at strong coupling and we compute it through the holographic approach [5-8].

In quantum field theories, extracting information about the model from the entanglement entropy of certain kinds of domains is an important task. The number of spacetime dimensions plays a central role in this analysis.

In two dimensional conformal field theories on the infinite line at zero temperature, when $A$ is an interval of length $\ell$, it is well known that the expansion of the entanglement entropy reads $S_{A}=(c / 3) \log (\ell / \varepsilon)+O(1)$ as the ultraviolet (UV) cutoff $\varepsilon \rightarrow 0^{+}$, being $c$ the central charge of the model [9-12]. Instead, when $A$ is made by disjoint intervals, the corresponding $S_{A}$ contains information also about the spectrum of the model [13-16].

In a three dimensional conformal field theory $\left(\mathrm{CFT}_{3}\right)$, considering a two dimensional spatial region $A \subsetneq \mathbb{R}^{2}$ whose boundary $\partial A$ is a smooth curve (which might be made by disjoint components), the expansion of the entanglement entropy as $\varepsilon \rightarrow 0^{+}$reads $S_{A}=b P_{A} / \varepsilon+O(1)$, where $P_{A}$ is the perimeter of $A$ and $b$ is a non universal and model dependent coefficient. The leading linear divergence corresponds to the area law term of the entanglement entropy in three spacetime dimensions [17-19]. When $A$ is a disk, it has been shown that the $O(1)$ term of this expansion behaves monotonically along the RG flow [20-25]. For a $\mathrm{CFT}_{3}$ with a holographic dual description, the holographic computation of $S_{A}$ at strong coupling coincides with the holographic computation of the expectation value of a spatial Wilson loop whose contour is $\partial A[26]$.

We are interested in two dimensional spatial regions $A \subsetneq \mathbb{R}^{2}$ which contain some isolated corners, namely such that $\partial A$ has a finite number of isolated singular points (vertices) 
separated by distances much larger than the UV cutoff. In these cases, besides the linearly diverging area law term, the expansion of the entanglement entropy includes also a subleading logarithmic divergence [27-32]

$$
S_{A}=b \frac{P_{A}}{\varepsilon}-\tilde{f}_{\text {tot }} \log \left(P_{A} / \varepsilon\right)+O(1)
$$

where the coefficient $\tilde{f}_{\text {tot }}$ is obtained by summing the contributions from all the corners occurring in $A$. It is not difficult to construct regions whose boundaries contain vertices from which an arbitrary even number of lines depart. For the sake of simplicity, we consider only vertices where either two or four lines join together. A vertex $V$ belonging to the former class is characterised by an opening angle $\theta$, while a vertex $W$ in the latter class is described by a vector of opening angles $\vec{\phi}_{W}$ made by three components. In the example shown in the left panel of figure 1 , the curve $\partial A$ contains two vertices of the first kind and one vertex of the second kind. For the domains containing these kinds of corner, $\tilde{f}_{\text {tot }}$ is given by

$$
\tilde{f}_{\text {tot }}=\sum_{V_{k}} \tilde{f}\left(\theta_{V_{k}}\right)+\sum_{W_{r}} \widetilde{\mathrm{F}}\left(\vec{\phi}_{W_{r}}\right)
$$

When $A$ has corners, we call corner functions all the functions of the opening angles in the sums occurring in the coefficient of the logarithmic divergence in $S_{A}$, whenever it is due only to the corners. In the case of (1.3), the corner functions are $\tilde{f}$ and $\widetilde{\mathrm{F}}$. Other corner functions will be discussed in the following. The corner function $\tilde{f}(\theta)$ is constrained by some important properties of the entanglement entropy. For instance, the fact that $S_{A}=S_{B}$ for pure states implies that $\tilde{f}(\theta)=\tilde{f}(2 \pi-\theta)$ and this tells us that the corner function vanishes quadratically $\tilde{f}(\theta)=\tilde{\sigma}(\pi-\theta)^{2}+\ldots$ when $\theta \rightarrow \pi$. Other interesting features of $\tilde{f}(\theta)$ (e.g. $\tilde{f}^{\prime \prime}(\theta) \geqslant 0$ ) can be derived from the strong subadditivity of the entanglement entropy [30, 33].

Besides the analysis of the corner functions $\tilde{f}(\theta)$ for specific quantum field theories [2832], many interesting results have been obtained in particular lattice models [34-39].

The corner function $\tilde{f}(\theta)$ depends on the underlying $\mathrm{CFT}_{3}$ model. However, it has been recently found that, by considering the coefficient $\tilde{\sigma}$ and the constant $C_{T}$ characterising the two point function of the stress tensor in the same $\mathrm{CFT}_{3}$, the relation $\tilde{\sigma} / C_{T}=\pi^{2} / 24$ holds for any $\mathrm{CFT}_{3}[40-42]$.

In this manuscript we are interested in a conformal field theory with a boundary (BCFT). In two spacetime dimensions, the conformal field theories with boundaries have been largely studied [43-46], but in higher dimensions much less is known [47, 48]. In this work we mainly focus on the three dimensional case $\left(\mathrm{BCFT}_{3}\right)$. The space of the boundary conditions which preserve the conformal invariance depends on the underlying model. In $\mathrm{BCFT}_{3}$, the presence of the boundary leads to a Weyl anomaly which is non vanishing only on the boundary and this allows to introduce two boundary charges $[49,50]$. In $\mathrm{BCFT}_{4}$, the Weyl anomaly is the sum of a well known term on the bulk and a term due to the occurrence of the boundary [49-52]. Other recent related results are discussed in [53, 54].

In this manuscript we mainly consider a $\mathrm{BCFT}_{3}$ whose boundary is flat; therefore any constant time slice of the spacetime is the half plane which can be described by the Cartesian coordinates $(x, y) \in \mathbb{R}^{2}$ with $x \geqslant 0$. In this $\mathrm{BCFT}_{3}$ setup, we study the entanglement 

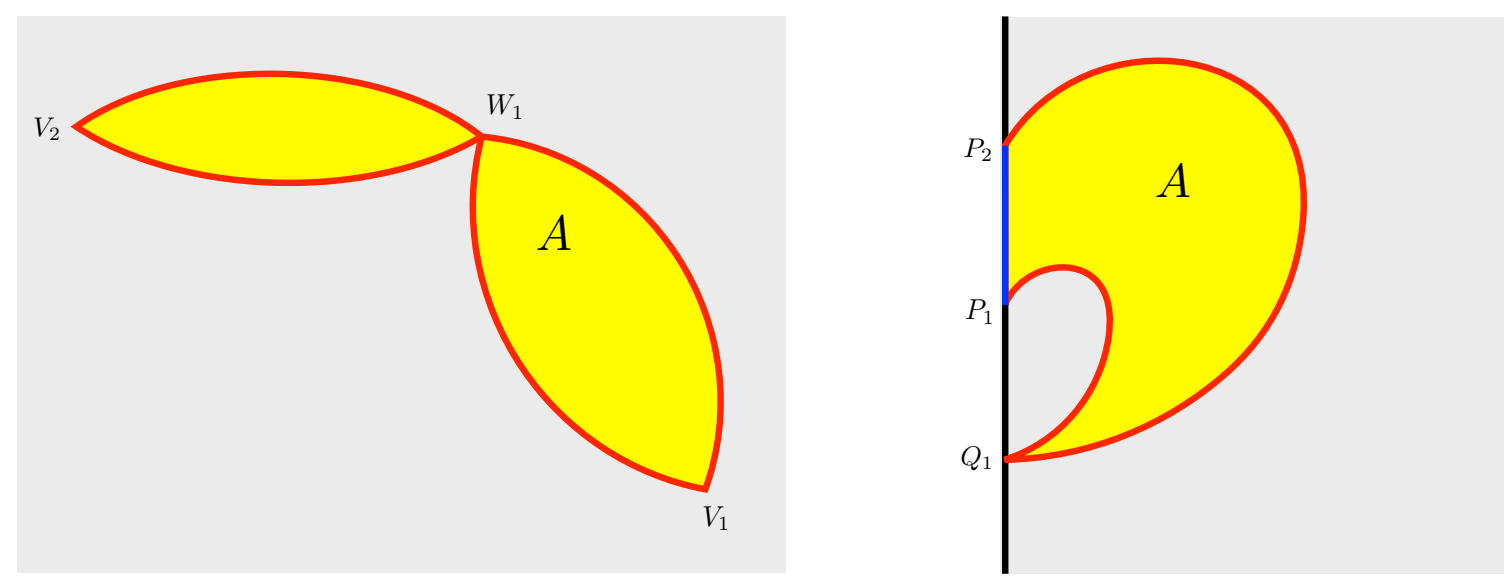

Figure 1. Examples of finite two dimensional regions $A$ (yellow domains) containing the kinds of corners considered in this manuscript. Left: $A$ is a domain in the plane with three corners and two different kinds of vertices. Right: $A$ is a domain in the half plane with three corners whose boundary $\partial A$ intersects the boundary of the $\mathrm{BCFT}_{3}$ (solid black line). The three vertices in $\partial A$ are also on the boundary of the $\mathrm{BCFT}_{3}$ and they belong to two different classes of vertices. In both panels, the red curve corresponds to the entangling curve $\partial A \cap \partial B$, whose length provides the area law term in (1.2) and in (1.4).

entropy of two dimensional regions $A$ whose boundaries $\partial A$ contain some isolated vertices which are all located on the boundary of the spatial half plane, which is the straight line at $x=0$. A prototypical example is the yellow domain in the right panel of figure 1 . Like for the previous regions, the distances between the vertices must be much larger than the UV cutoff. For this kind of domains, the expansion of the entanglement entropy as $\varepsilon \rightarrow 0^{+}$reads

$$
S_{A}=b \frac{P_{A, B}}{\varepsilon}-f_{\alpha, \text { tot }} \log \left(P_{A, B} / \varepsilon\right)+O(1)
$$

where $P_{A, B} \equiv$ length $(\partial A \cap \partial B)$ is the length of the curve shared by $\partial A$ and the boundary $\partial B$ of its complement (the red curve in the right panel of figure 1), which is often called entangling curve. Thus, $P_{A, B} \leqslant P_{A}$ and $P_{A}-P_{A, B}$ is the length of $\partial A_{\mathrm{bdy}} \equiv \partial A \cap\{(x, y) \mid x=0\}$ (in the right panel of figure 1 we have $\partial A_{\mathrm{bdy}}=\overline{P_{1} P_{2}} \cup\left\{Q_{1}\right\}$ ). This is a consequence of the relation $S_{A}=S_{B}$, which holds whenever the whole system is in a pure state. The coefficient of the leading divergence in (1.4) is the same that occurs in the leading divergence of (1.2) because it is related to some local effects close to the entangling curve; therefore it should be independent of the occurrence of a boundary. Instead, the coefficient $f_{\alpha, \text { tot }}$ of the logarithmic divergence in (1.4) is expected to depend on the boundary conditions characterising the $\mathrm{BCFT}_{3}$ in a highly non trivial way. The index $\alpha$ labels the boundary conditions allowed by the conformal invariance in the underlying model.

Domains $A$ whose boundaries contain vertices on the $x=0$ line from which an arbitrary even number of lines belonging to $\partial A$ depart can be easily drawn. For the sake of simplicity, we restrict our analysis to vertices of $\partial A$ on the $x=0$ line where only two lines of $\partial A$ (the edges of the corner) join together. Given a vertex belonging to this class, there are two possibilities: either one edge or none of the two edges is on the boundary. In the 
former case we denote the vertex by $P$ and the corner is characterised only by an angle $\gamma$, while in the latter case we label the vertex with $Q$ and the corresponding corner in $A$ is characterised by a pair $\vec{\omega}$ of opening angles. An example of domain $A$ containing these two kinds of corners is shown in the right panel of figure 1 , where $\partial A$ has two vertices $P_{1}$ and $P_{2}$ of the first kind and one vertex $Q_{1}$ of the second kind.

For this class of regions $A$, the coefficient $f_{\alpha, \text { tot }}$ of the logarithmic divergence in (1.4) is obtained by summing the contributions of the all corners on the boundary, namely

$$
f_{\alpha, \text { tot }}=\sum_{P_{i}} f_{\alpha}\left(\gamma_{P_{i}}\right)+\sum_{Q_{j}} \mathrm{~F}_{\alpha}\left(\vec{\omega}_{Q_{j}}\right)
$$

where $f_{\alpha}$ and $\mathrm{F}_{\alpha}$ are corner functions which depend on the boundary conditions of the $\mathrm{BCFT}_{3}$. Interesting results about $f_{\alpha}(\gamma)$ have been obtained in [55-57].

One can easily consider a more general class of domains $A$ whose boundaries contain also vertices of the types introduced above which have $x>0$. In these cases the area law term of the entanglement entropy is like the one in (1.4) and the coefficient of the logarithmic divergence is simply the sum of (1.3) and (1.5).

In this manuscript we are interested in the corner functions occurring in (1.5) for a $\mathrm{BCFT}_{3}$ at strong coupling. The main tool employed in our analysis is the gauge/gravity correspondence and, in particular, the holographic prescription to compute the entanglement entropy.

Let us consider a $\mathrm{CFT}_{d+1}$ in $d+1$ spacetime dimensions which has a gravitational dual description through an asymptotically $\mathrm{AdS}_{d+2}$ spacetime. In the Poincaré coordinates, denoting by $z$ the extra dimension of the gravitational theory with respect to the $d+1$ dimensions of the $\mathrm{CFT}_{d+1}$, the boundary of the gravitational spacetime, where the $\mathrm{CFT}_{d+1}$ is defined, corresponds to $z=0$. For the static cases, the entanglement entropy of a spatial region $A$ in a $t=$ const slice of the $\mathrm{CFT}_{d+1}$ at strong coupling is given by the holographic formula $[5,6]$ (see [4] for a recent review)

$$
S_{A}=\frac{\mathcal{A}_{A}}{4 G_{\mathrm{N}}}
$$

where $G_{\mathrm{N}}$ is the $d+2$ dimensional gravitational Newton constant and $\mathcal{A}_{A}$ is the area of the $d$ dimensional minimal area hypersurface $\hat{\gamma}_{A}$ anchored to the boundary of $A$, namely such that $\partial \hat{\gamma}_{A}=\partial A$. Since the asymptotically $\mathrm{AdS}_{d+2}$ gravitational background is a non compact space and $\hat{\gamma}_{A}$ reaches its boundary, the area of $\hat{\gamma}_{A}$ diverges. In order to regulate the area $\mathcal{A}_{A}$, we have to introduce a cutoff $z \geqslant \varepsilon>0$ in the holographic direction $z$ such that $\varepsilon \ll P_{A}$. According to the AdS/CFT dictionary, $\varepsilon$ is the gravitational dual of the UV cutoff in the $\mathrm{CFT}_{d+1}$. Denoting by $\hat{\gamma}_{\varepsilon} \equiv \hat{\gamma}_{A} \cap\{z \geqslant \varepsilon\}$ the restriction of $\hat{\gamma}_{A}$ to $z \geqslant \varepsilon$, in (1.6) one has to consider $\mathcal{A}_{A}=\mathcal{A}\left[\hat{\gamma}_{\varepsilon}\right]$ and then expand the resulting expression for $\varepsilon \rightarrow 0^{+}$. The terms of this expansion can be compared with the ones occurring in the expansion of $S_{A}$ computed through CFT techniques. Various consistency checks of (1.6) has been done (e.g. the strong subadditivity property $[33,58])$ and nowadays the holographic formula (1.6) is largely recognised as a tool to evaluate the entanglement entropy in the strong coupling regime of CFTs with a gravitational dual description. In this manuscript we are mainly interested in the $d=2$ case, but some results are obtained for a generic $d$. 
The holographic prescription (1.6) has been applied also for domains $A$ whose boundaries contain singular points $[30,59-62]$. We are interested in the $d=2$ case, where the expansion of $\mathcal{A}\left[\hat{\gamma}_{\varepsilon}\right]$ as $\varepsilon \rightarrow 0^{+}$for domains $A$ with corners reads

$$
\mathcal{A}\left[\hat{\gamma}_{\varepsilon}\right]=L_{\text {AdS }}^{2}\left(\frac{P_{A}}{\varepsilon}-\widetilde{F}_{\text {tot }} \log \left(P_{A} / \varepsilon\right)+O(1)\right)
$$

being $L_{\mathrm{AdS}}$ the AdS radius of the gravitational background. Considering the two classes of vertices discussed below (1.2), the coefficient of the logarithmic divergence in (1.7) reads

$$
\widetilde{F}_{\text {tot }}=\sum_{V_{k}} \widetilde{F}\left(\theta_{V_{k}}\right)+\sum_{W_{r}} \widetilde{\mathcal{F}}\left(\vec{\phi}_{W_{r}}\right)
$$

Comparing (1.2) and (1.3) with (1.6), (1.7) and (1.8), we have that the holographic corner functions are proportional to $\widetilde{F}(\theta)$ and $\widetilde{\mathcal{F}}(\vec{\phi})$ through the positive constant $\frac{L_{\mathrm{AdS}}^{2}}{4 G_{\mathrm{N}}}$. Nonetheless, in the following we mainly refer to the latter ones as the holographic corner functions, unless stated otherwise. The analytic expression of the corner function $\widetilde{F}(\theta)$ has been found in [27]. The corner function $\widetilde{\mathcal{F}}(\vec{\phi})$ can be easily written once $\widetilde{F}(\theta)$ is known [62].

In this manuscript we study the corner functions $f_{\alpha}(\gamma)$ and $\mathrm{F}_{\alpha}(\vec{\omega})$ for a $\mathrm{BCFT}_{3}$ at strong coupling, assuming that a holographic dual exists. We consider the $\mathrm{AdS}_{d+2} / \mathrm{BCFT}_{d+1}$ setup introduced in [63] and further developed in [64, 65], where the $d+2$ dimensional bulk is limited by the occurrence of a $d+1$ dimensional hypersurface $\widetilde{\mathcal{Q}}$ in the bulk which has the same boundary of the $\mathrm{BCFT}_{d+1}$. Also previous works have considered the occurrence of a hypersurface in the bulk [66-69]. An interesting application of the AdS/BCFT setup has been discussed in [70, 71]. The boundary conditions determining $\widetilde{\mathcal{Q}}$ are crucial in the construction of [63-65]. A setup of the correspondence based on a different boundary condition has been recently suggested [72-75].

In this manuscript we mainly focus on the simplest situation of a $\mathrm{BCFT}_{3}$ with a flat boundary. In this case $\widetilde{\mathcal{Q}}=\mathcal{Q}$ is the same half hyperplane for both the prescriptions mentioned above. Its slope $\alpha$ is related to a real parameter occurring in the boundary term of the gravitational action in the bulk.

In a $\mathrm{BCFT}_{3}$ with a holographic dual description, we consider two dimensional domains $A$ described above, namely the ones with isolated corners whose vertices belong to the boundary of the $\mathrm{BCFT}_{3}$. For these domains the entanglement entropy is given by (1.4) and (1.5). By employing the holographic formula (1.6) properly adapted to the AdS/BCFT setup, we find

$$
\mathcal{A}\left[\hat{\gamma}_{\varepsilon}\right]=L_{\text {AdS }}^{2}\left(\frac{P_{A, B}}{\varepsilon}-F_{\alpha, \text { tot }} \log \left(P_{A, B} / \varepsilon\right)+O(1)\right)
$$

where $P_{A, B} \leqslant P_{A}$ is the length of the entangling curve in the boundary at $z=0$. We are mainly interested in the coefficient of the logarithmic divergence, which given by the sum of the contributions from all the vertices of $\partial A$, namely

$$
F_{\alpha, \text { tot }}=\sum_{P_{i}} F_{\alpha}\left(\gamma_{P_{i}}\right)+\sum_{Q_{j}} \mathcal{F}_{\alpha}\left(\omega_{Q_{j}}, \gamma_{Q_{j}}\right)
$$


where the functions occurring in the sums depend on the slope $\alpha$ of the half plane $\mathcal{Q}$. We mainly refer to $F_{\alpha}(\gamma)$ and $\mathcal{F}_{\alpha}(\omega, \gamma)$ as the holographic corner functions in the presence of a boundary, although the proportionality constant $\frac{L_{\mathrm{AdS}}^{2}}{4 G_{\mathrm{N}}}$ should be taken into account.

In this manuscript we find analytic expressions for the corner functions $F_{\alpha}(\gamma)$ and $\mathcal{F}_{\alpha}(\omega, \gamma)$. Numerical checks of these results are performed by constructing the minimal area surfaces corresponding to some finite domains containing corners. In the numerical analysis we have employed Surface Evolver [76, 77] to construct the minimal area surfaces, a software which has been already used in $[60,61]$ to study the shape dependence of the holographic entanglement entropy in $\mathrm{AdS}_{4} / \mathrm{CFT}_{3}$.

We also compute the holographic entanglement entropy of an infinite strip, which can be either adjacent or parallel to the boundary. This is done because the result for the infinite strip adjacent to the boundary can be related to the limit $\gamma \rightarrow 0$ of the corner function $F_{\alpha}(\gamma)$. The holographic entanglement entropy of an infinite strip adjacent or parallel to the boundary is computed also for a generic number of spacetime dimensions.

We find it worth mentioning here that, within the $\mathrm{AdS}_{4} / \mathrm{BCFT}_{3}$ setup of [63], we find

a proportionality relation between the coefficient $f_{\alpha}^{\prime \prime}(\pi / 2)=\frac{L_{\mathrm{AdS}}^{2}}{4 G_{\mathrm{N}}} F_{\alpha}^{\prime \prime}(\pi / 2)$ occurring in the expansion of the holographic corner function when $\gamma \rightarrow \pi / 2$ and the holographic result for a coefficient which characterises the behaviour of the one point function of the stress tensor in the proximity of the curved boundary of a $\mathrm{BCFT}_{3}$.

The paper is organised as follows. In section 2 the strong subadditivity is employed to find constraints for the corner functions $f_{\alpha}(\gamma)$ and $\mathrm{F}_{\alpha}(\omega, \gamma)$ in a $\mathrm{BCFT}_{3}$. In section 3 we test our numerical approach based on Surface Evolver on the corner functions in $\mathrm{AdS}_{4} / \mathrm{CFT}_{3}$. This is a good benchmark for our method because well known analytic expressions are available for the corner functions occurring in (1.8). The AdS/BCFT setup is briefly reviewed in section 4 , where we also discuss the prescription to compute the holographic entanglement entropy of a domain with generic shape. This prescription for the holographic entanglement entropy is applied for two simple domains in section 5: the half disk centered on the boundary and the infinite strip, which can be either adjacent or parallel to the boundary at finite distance from it. In section 6 we describe the main result of this manuscript, namely the holographic entanglement entropy of the infinite wedge adjacent to the boundary, which provides the analytic expression of the corner function $F_{\alpha}(\gamma)$. In section 7 this corner function is employed to find an analytic formula for the corner function $\mathcal{F}_{\alpha}(\omega, \gamma)$. Some conclusions and open problems are discussed in section 8 .

The main text of this manuscript contains only the description of the main results. All the computational details underlying their derivations and also some generalisations to an arbitrary number of spacetime dimensions have been collected and discussed in the appendices A, B, C, D, E, F, G and H.

\section{Constraining the corner functions}

In this section we employ the strong subadditivity of the entanglement entropy [33] to constrain the corner functions introduced in (1.5). Our analysis is similar to the one performed in [30] for the corner function $\tilde{f}(\theta)$ in $(1.3)$. 

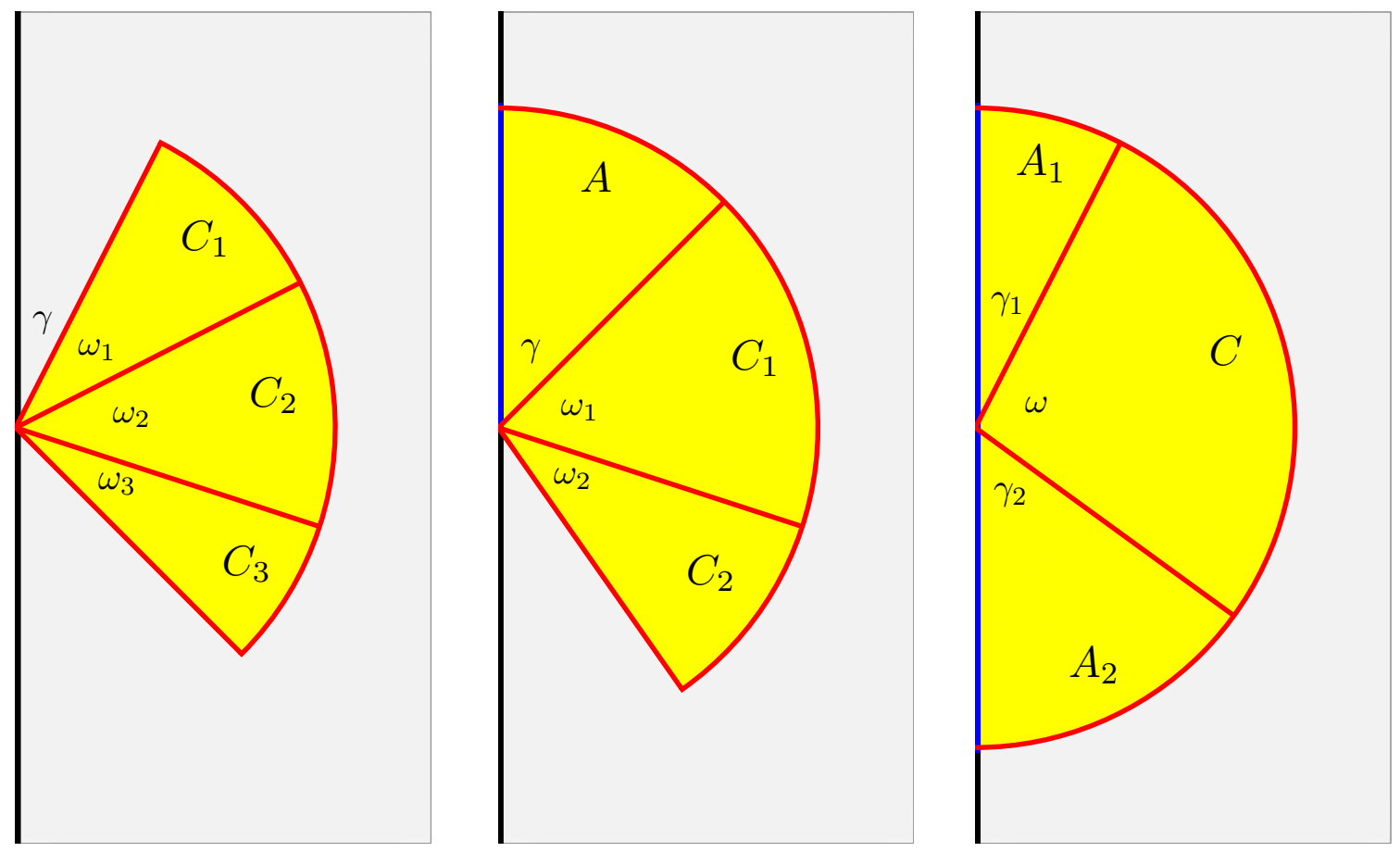

Figure 2. Configurations of adjacent domains containing corners (yellow regions) in the half plane $x \geqslant 0$ (grey region) which have been used in section 2 to constrain the corner functions through the strong subadditivity.

Let us consider a $\mathrm{BCFT}_{3}$ in its ground state and the domain $A$ given by the infinite wedge adjacent to the boundary whose opening angle is $\gamma$. The complementary domain $B$ is the infinite wedge adjacent to the boundary sharing with $A$ its edge which is not on the boundary and its opening angle is $\pi-\gamma$. Since the ground state is a pure state, we have $S_{A}=S_{B}$. Combining this property with (1.2) specialised to these complementary domains, we have

$$
f_{\alpha}(\pi-\gamma)=f_{\alpha}(\gamma)
$$

namely the corner function $f_{\alpha}(\gamma)$ is symmetric with respect to $\gamma=\pi / 2$; therefore we are allowed to study this corner function for $0<\gamma \leqslant \pi / 2$. Hereafter we mainly consider $\gamma \in(0, \pi / 2]$ for the argument of this corner function. Nonetheless, whenever $\gamma \in(0, \pi)$ in the following, we always mean $f_{\alpha}(\gamma)=f_{\alpha}(\min [\gamma, \pi-\gamma])$.

By assuming that $f_{\alpha}(\gamma)$ is smooth for $\gamma \in(0, \pi)$, the symmetry (2.1) implies that its expansion around $\gamma=\pi / 2$ includes only even powers of $\gamma-\pi / 2$, namely

$$
f_{\alpha}(\gamma)=f_{\alpha}(\pi / 2)+\frac{f_{\alpha}^{\prime \prime}(\pi / 2)}{2}(\gamma-\pi / 2)^{2}+\ldots \quad \gamma \rightarrow \frac{\pi}{2}
$$

In the remaining part of this section we discuss some constraints for the corner functions in (1.5) obtained by imposing that the strong subadditivity of the entanglement entropy is valid for particular configurations of adjacent domains. 
Consider the configuration of adjacent regions shown in the left panel of figure 2. The strong subadditivity inequality specialised to this case states that

$$
S_{C_{1} \cup C_{2}}+S_{C_{2} \cup C_{3}} \geqslant S_{C_{1} \cup C_{2} \cup C_{3}}+S_{C_{2}}
$$

By employing the expressions (1.4) and (1.5), which provide the entanglement entropy of the domains occurring in this inequality, one observes that the area law terms and the logarithmic divergencies corresponding to vertices which are not on the boundary simplify. The remaining terms at leading order provide the following inequality

$$
F_{\alpha}\left(\omega_{1}+\omega_{2}+\omega_{3}, \gamma\right)-F_{\alpha}\left(\omega_{1}+\omega_{2}, \gamma\right) \geqslant F_{\alpha}\left(\omega_{2}+\omega_{3}, \gamma+\omega_{1}\right)-F_{\alpha}\left(\omega_{2}, \gamma+\omega_{1}\right)
$$

Multiplying both sides of this inequality by $1 / \omega_{3}>0$ first and then taking the limit $\omega_{3} \rightarrow 0^{+}$, one finds

$$
\partial_{\omega} F_{\alpha}\left(\omega_{2}+\omega_{1}, \gamma\right) \geqslant \partial_{\omega} F_{\alpha}\left(\omega_{2}, \gamma+\omega_{1}\right)
$$

Next we add $-\partial_{\omega} F_{\alpha}\left(\omega_{2}, \gamma\right)$ to both sides of $(2.5)$, then we multiply them by $1 / \omega_{1}>0$ and finally take the limit $\omega_{1} \rightarrow 0^{+}$. The resulting inequality reads

$$
\partial_{\omega}^{2} F_{\alpha}(\omega, \gamma) \geqslant \partial_{\omega} \partial_{\gamma} F_{\alpha}(\omega, \gamma)
$$

This property resembles to $\tilde{f}^{\prime \prime}(\omega) \geqslant 0$ for the corner function $\tilde{f}(\omega)$ in $\mathrm{CFT}_{3}$ [30].

The second configuration of adjacent domains that we consider is the one depicted in the middle panel of figure 2. In this case, the constraint given by the strong subadditivity reads $S_{A \cup C_{1}}+S_{C_{1} \cup C_{2}} \geqslant S_{A \cup C_{1} \cup C_{2}}+S_{C_{1}}$ and simplifications similar to the ones discussed in the previous case occur. In particular, the leading non vanishing terms correspond to the vertex shared by the three domains. The resulting inequality reads

$$
f_{\alpha}\left(\gamma+\omega_{1}+\omega_{2}\right)-f_{\alpha}\left(\gamma+\omega_{1}\right) \geqslant \mathrm{F}_{\alpha}\left(\omega_{1}+\omega_{2}, \gamma\right)-\mathrm{F}_{\alpha}\left(\omega_{1}, \gamma\right)
$$

Multiplying both sides of this relation by $1 / \omega_{1}>0$ and taking the limit $\omega_{1} \rightarrow 0^{+}$, one obtains

$$
\partial_{\omega} \mathrm{F}_{\alpha}(\omega, \gamma) \leqslant \partial_{\gamma} f_{\alpha}(\gamma+\omega)
$$

Let us study also the configuration shown in the right panel of figure 2 , where $\gamma_{1}+\omega+$ $\gamma_{2}=\pi$ and the strong subadditivity property provides the constraint $S_{A_{1} \cup C}+S_{A_{2} \cup C} \geqslant$ $S_{A_{1} \cup A_{2} \cup C}+S_{C}$. By using (1.4) and (1.5) as done in the previous cases, we get another inequality among the corner functions corresponding to the vertex shared by the three adjacent domains

$$
f_{\alpha}\left(\gamma_{1}+\omega\right)+f_{\alpha}\left(\gamma_{2}+\omega\right) \leqslant \mathrm{F}_{\alpha}\left(\omega, \gamma_{1}\right) \quad \gamma_{1} \leqslant \gamma_{2}
$$

Since $\gamma_{2}+\omega=\pi-\gamma_{1}$, we can employ (2.1), finding that (2.9) can be written as

$$
f_{\alpha}(\gamma+\omega)+f_{\alpha}(\gamma) \leqslant \mathrm{F}_{\alpha}(\omega, \gamma) \quad \gamma \leqslant \frac{\pi-\omega}{2}
$$

We remark that the constraints (2.6), (2.8) and (2.10) hold whenever the entanglement entropy is given by (1.4) and (1.5), with corner functions which are regular enough to define the derivatives occurring in these inequalities. 


\section{Warming up: corner functions in $\mathrm{AdS}_{4} / \mathrm{CFT}_{3}$}

In this section we consider the holographic entanglement entropy of domains with corners in $\mathrm{AdS}_{4} / \mathrm{CFT}_{3}$. The aim of this analysis is to test our numerical approach to the corner functions for the holographic entanglement entropy on the well known case of $\mathrm{AdS}_{4} / \mathrm{CFT}_{3}$, in order to apply it to the corner functions in $\mathrm{AdS}_{4} / \mathrm{BCFT}_{3}$ in the following sections. Our numerical data are obtained by employing Surface Evolver [76, 77], a software developed by Ken Brakke, and the method is briefly discussed in the appendix A.

Given a $\mathrm{CFT}_{3}$ in the three dimensional Minkowski space parameterised by $(t, x, y)$ which has a dual holographic description, the bulk metric dual to its ground state is $\mathrm{AdS}_{4}$. In Poincaré coordinates $(t, z, x, y)$, the metric induced on a constant time slice of $\mathrm{AdS}_{4}$ is

$$
d s^{2}=\frac{L_{\mathrm{AdS}}^{2}}{z^{2}}\left(d z^{2}+d x^{2}+d y^{2}\right) \quad z>0
$$

where $L_{\text {AdS }}$ is the AdS radius. The metric (3.1) characterises the three dimensional hyperbolic space $\mathbb{H}_{3}=\left.A d S_{4}\right|_{t=\text { const }}$.

The holographic entanglement entropy (1.6) of a two dimensional domain $A$ in the plane $(x, y) \in \mathbb{R}^{2}$ is obtained by finding the minimal area surface $\hat{\gamma}_{A}$ anchored to $\partial A$ first and then computing the area of $\hat{\gamma}_{\varepsilon}=\hat{\gamma}_{A} \cap\{z \geqslant \varepsilon\}$ for $\varepsilon \rightarrow 0^{+}$. When $\partial A$ contains isolated vertices and each of them has an arbitrary even number of edges, the expansion of the area of $\hat{\gamma}_{\varepsilon}$ is given by (1.7).

The analytic expression for the coefficient $\widetilde{F}_{\text {tot }}$ of the logarithmic divergence can be found by using the corner function $\widetilde{F}(\theta)$ found in [27]. This function reads

$$
\widetilde{F}(\theta) \equiv 2 F\left(q_{0}\right)
$$

where

$$
F\left(q_{0}\right) \equiv \frac{\mathbb{E}\left(\tilde{q}_{0}^{2}\right)-\left(1-\tilde{q}_{0}^{2}\right) \mathbb{K}\left(\tilde{q}_{0}^{2}\right)}{\sqrt{1-2 \tilde{q}_{0}^{2}}}
$$

and the opening angle $\theta$ of the wedge is given by

$$
\frac{\theta}{2}=\tilde{q}_{0} \sqrt{\frac{1-2 \tilde{q}_{0}^{2}}{1-\tilde{q}_{0}^{2}}}\left[\Pi\left(1-\tilde{q}_{0}^{2}, \tilde{q}_{0}^{2}\right)-\mathbb{K}\left(\tilde{q}_{0}^{2}\right)\right] \equiv P_{0}\left(q_{0}\right)
$$

where the positive parameter $\tilde{q}_{0} \in(0,1 / 2)$ is related to a positive parameter $q_{0}$ as

$$
\tilde{q}_{0}^{2} \equiv \frac{q_{0}^{2}}{1+2 q_{0}^{2}} \quad q_{0}>0
$$

The geometric meaning of $q_{0}$ will be discussed in section 6 . The functions $\mathbb{K}(m), \mathbb{E}(m)$ and $\Pi(n, m)$ are the complete elliptic integrals of the first, second and third kind respectively. From (3.2) and (3.4), one can plot the curve $\widetilde{F}(\theta)$ parametrically in terms of $q_{0}>0$, finding the blue curve shown in figure 4 .

Since $S_{A}=S_{B}$ for pure states, for the argument of the corner function $\widetilde{F}(\theta)$ we have $\theta \in(0, \pi]$. Hereafter, whenever $\theta \in(0,2 \pi)$ we mean $\widetilde{F}(\theta)=\widetilde{F}(\min [\theta, 2 \pi-\theta])$. 


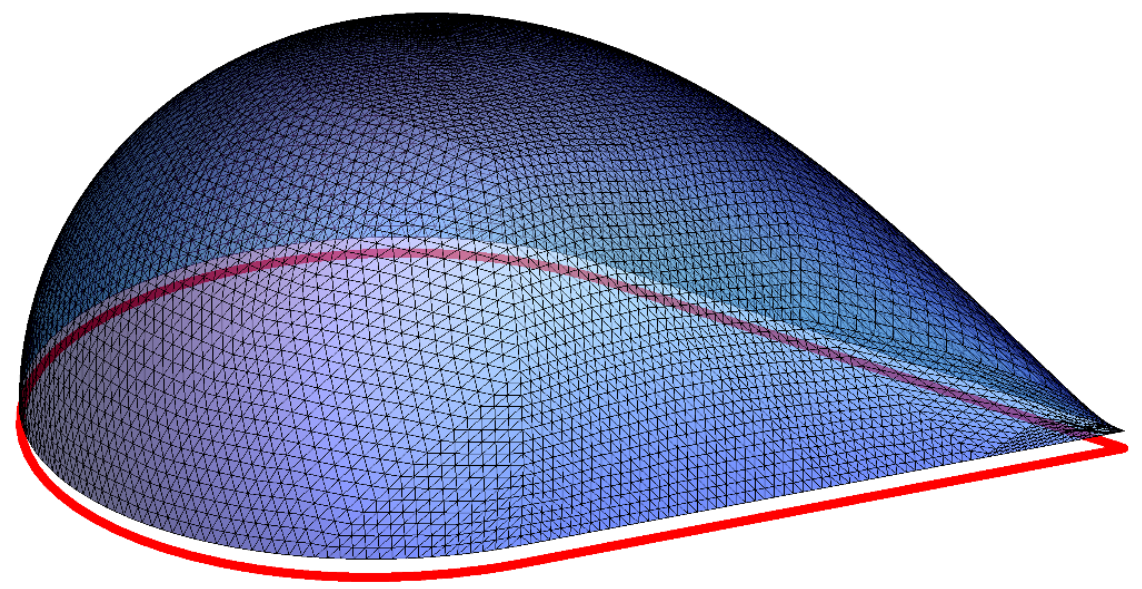

Figure 3. Triangulated surface in $\mathbb{H}_{3}$ which approximates the minimal area surface $\hat{\gamma}_{A}$ corresponding to a single drop region $A$ in the $z=0$ plane, as discussed in section 3.1. The boundary $\partial A$ (red curve) lies in the $z=0$ plane and it is characterised by $L=1$ and $\theta=\pi / 3$. The UV cutoff is $\varepsilon=0.03$. The triangulation has been obtained with Surface Evolver by setting $\partial A$ at $z=\varepsilon$.

In the remaining part of this section we study two simple domains whose holographic entanglement entropy is given by (1.7) and (1.8). In the first example $\partial A$ has a single vertex with two edges and the second case $\partial A$ has a single vertex with four edges. Thus, only one term occurs in (1.8) specialised to these domains.

\subsection{Single drop}

The first simply connected finite domain $A$ that we consider is similar to a two dimensional drop. It is constructed by taking the infinite wedge with opening angle $\theta<\pi$ (whose tip is denoted by $P$ ) and the disk of radius $R$ which is tangent to both the edges of the wedge. The distance between the two intersection points and $P$ is $L=R \cot (\theta / 2)$. Considering the circular sector given by the intersection of the infinite wedge with the disk centered in $P$ with radius $L$, our drop region $A$ is obtained as the union between this circular sector and the disk of radius $R$ tangent to the edges of the infinite wedge introduced above. This domain can be characterised by the parameters $L$ and $\theta$. Its boundary $\partial A$ is a smooth curve except for the vertex $P$, where two edges join, whose length is $P_{A}=2 L+R(\pi+\theta)$. An example of drop domain is the region in the plane enclosed by red curve in figure 3 .

The holographic entanglement entropy of a drop region $A$ in the $z=0$ plane is obtained by computing the area $\mathcal{A}\left[\hat{\gamma}_{\varepsilon}\right]$ from the minimal surface $\hat{\gamma}_{A}$ embedded in $\mathbb{H}_{3}$ which is anchored to $\partial A$, as prescribed by (1.6). The result is (1.7) with $\widetilde{F}_{\text {tot }}=\widetilde{F}(\theta)$, being $\widetilde{F}(\theta)$ the corner function given by (3.2) and (3.4). The main advantage of our choice for $A$ is that we can vary the opening angle $\theta$ in a straightforward way. The minimal area surfaces $\hat{\gamma}_{A}$ corresponding to regular polygons and other finite domains with three or more vertices have been studied in [60].

Finding analytic expressions for $\hat{\gamma}_{A}$ and for the area of $\hat{\gamma}_{\varepsilon}$ when $A$ is a finite region without particular symmetries is very difficult. We perform a numerical analysis by employing 


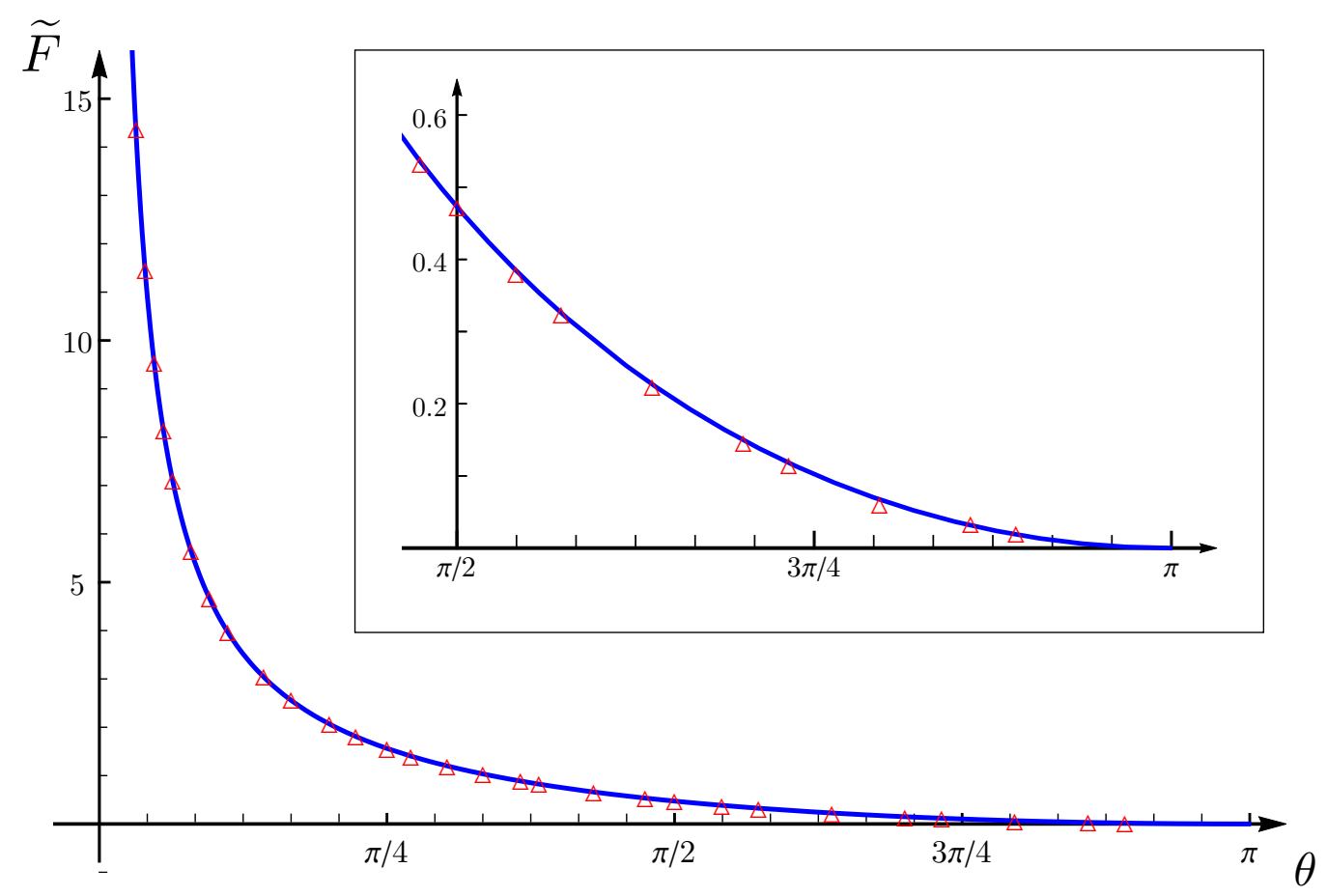

Figure 4. Corner function for a vertex with two edges in $\mathrm{AdS}_{4} / \mathrm{CFT}_{3}$. The blue curve corresponds to the analytic expression given by (3.2) and (3.4) found in [27]. The points labeled by the red triangles have been found through the numerical analysis based on Surface Evolver (see section 3.1 and the appendix A). The inset highlights the domain corresponding to opening angles close to $\pi$.

Surface Evolver, which provides approximate solutions for $\hat{\gamma}_{A}$ and the corresponding value for $\mathcal{A}\left[\hat{\gamma}_{\varepsilon}\right]$. In figure 3 we show a refined triangulation which approximates the minimal surface $\hat{\gamma}_{A}$ anchored to a single drop domain. Some technical details about the construction of this kind of triangulations are discussed in the appendix A (see also [60]).

As briefly explained in the appendix $\mathrm{A}$, by fitting the numerical data for $\mathcal{A}\left[\hat{\gamma}_{\varepsilon}\right]$ obtained for various $\varepsilon$ at fixed values of $\theta$ and $L$, we find a numerical value for the corner function which can be compared to the corresponding value coming from the analytic expression of $\widetilde{F}(\theta)$ given by (3.2) and (3.4). Repeating this analysis for different values of $\theta$, we have obtained the results shown in figure 4 , where the blue solid curve is the analytic curve $\widetilde{F}(\theta)$ found in [27], while the points marked by the red triangles have been found through our numerical analysis. The agreement is exceptionally good in the range of $\theta$ which has been explored.

\subsection{Two drops with the same tip}

The second region that we consider can be obtained as the union $A=A_{1} \cup A_{2}$ of two single drop regions $A_{1}$ and $A_{2}$, where $A_{1}$ and $A_{2}$ have the same tip $W$, which is also the only element of their intersection, i.e. $A_{1} \cap A_{2}=\{W\}$. The boundary $\partial A$ is smooth except at the vertex $W$, where four lines join together. Considering the four adjacent corners with the common vertex $W$, let us denote by $\phi_{1}$ and $\phi_{2}$ the opening angles of the corners in $A_{1}$ and $A_{2}$ respectively and by $\varphi_{1}$ and $\varphi_{2}$ the opening angles of the other two corners 

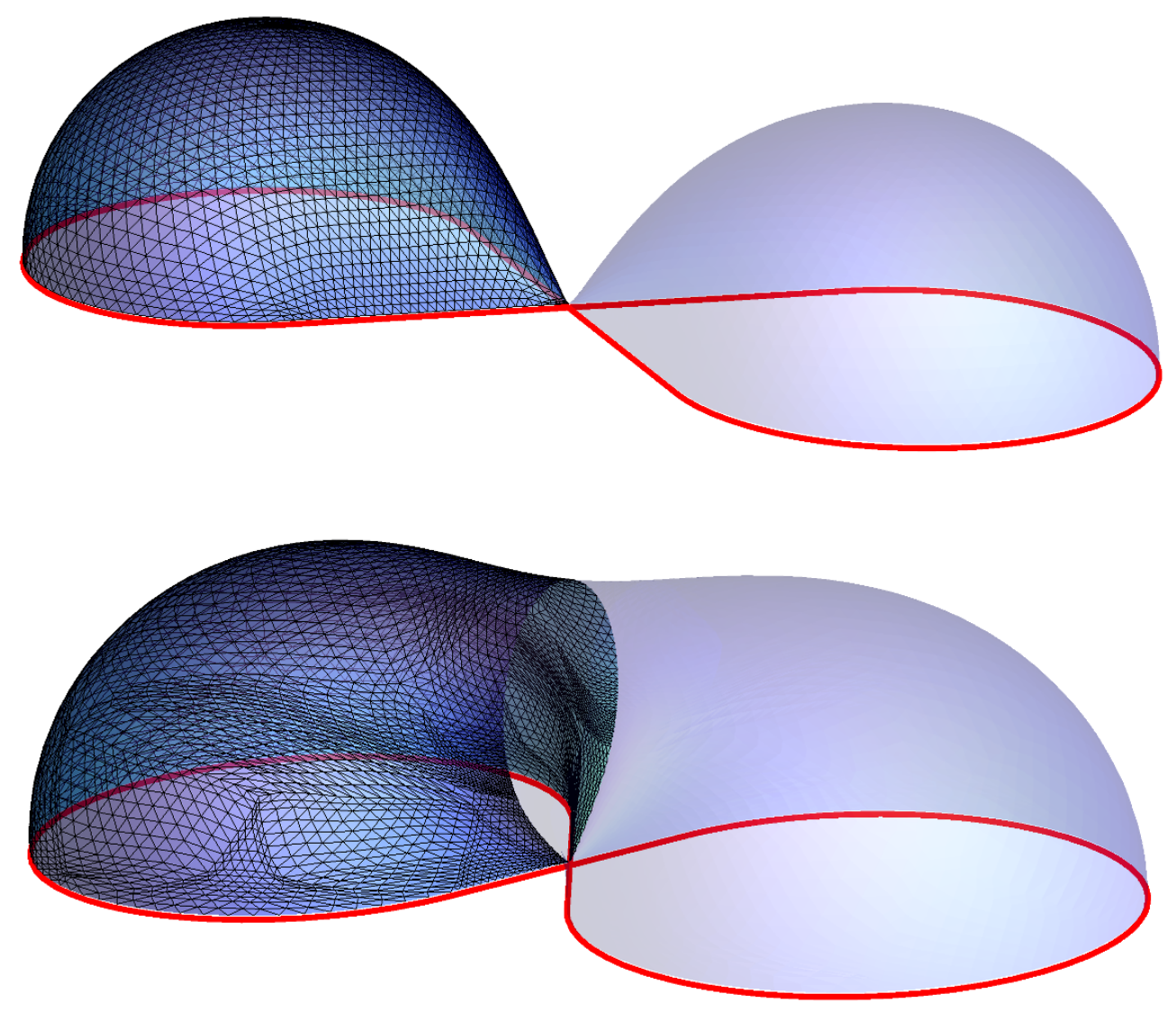

Figure 5. Triangulated surfaces in $\mathbb{H}_{3}$ approximating the minimal area surfaces $\hat{\gamma}_{A}$ which correspond to two different double drop regions $A$ described in section 3.2. For these domains $\phi_{1}=\phi_{2} \equiv \phi$ and $\varphi_{1}=\varphi_{2}=\pi-\phi$. The boundary $\partial A$ (red curve) belongs to the $z=0$ plane and the UV cutoff is $\varepsilon=0.03$. Top: $L=2$ and $\phi=1.4$ (below $\phi_{c}=\pi / 2$ ). Bottom: $L=1$ and $\phi=2.2\left(\right.$ above $\phi_{c}=\pi / 2$ ).

which do not belong to $A$. We can assume $0<\phi_{1} \leqslant \phi_{2}$ and $0<\varphi_{1} \leqslant \varphi_{2}$ without loss of generality. The configuration of the corners around $W$ can be characterised by the three angles $\vec{\phi}=\left(\phi_{1}, \varphi_{1}, \phi_{2}\right)$ because the remaining one can be determined by the consistency condition $\phi_{1}+\phi_{2}+\varphi_{1}+\varphi_{2}=2 \pi$, where $\phi_{1}<\pi$ and $\varphi_{1}<\pi$.

The holographic entanglement entropy for this "double drop" region $A$ can be found from the general expressions (1.7) and (1.8). The coefficient of the logarithmic divergence of $\mathcal{A}\left[\hat{\gamma}_{\varepsilon}\right]$ comes from the contribution of the vertex $W$ and it is given by $\widetilde{F}_{\text {tot }}=\widetilde{\mathcal{F}}(\vec{\phi})$.

Symmetric configurations can be considered by imposing constraints among the components of $\vec{\phi}$. For instance, we can study domains such that $A_{1}$ and $A_{2}$ coincide after a proper rotation of one of them. In this cases the configuration of the corners at the common tip $W$ is determined by two parameters: the opening angle $\phi_{1}=\phi_{2} \equiv \phi$ and the relative orientation given by $\varphi_{1}$. Let us stress that the coefficient of the logarithmic term is determined by the local configuration of corners around the vertex $W$ and it is not influenced by the shape of the entire domain $A$. 
We consider first the configuration where the two drop regions $A_{1}$ and $A_{2}$ are symmetric with respect to their common tip $W$. This means that $\phi_{1}=\phi_{2} \equiv \phi$ and also $\varphi_{1}=\varphi_{2} \equiv \varphi$. The resulting domain $A$ is symmetric w.r.t. two orthogonal straight lines whose intersection point is $W$. Since $\varphi+\phi=\pi$, the coefficient of the logarithmic divergence in (1.7) is determined only by the angle $\phi$ for these cases, namely $\widetilde{F}_{\text {tot }}=\widetilde{\mathcal{F}}(\phi)$. In particular, it is not difficult to realise that for these configurations the corner function is given by [62]

$$
\widetilde{\mathcal{F}}(\phi)=2 \max \{\widetilde{F}(\phi), \widetilde{F}(\pi-\phi)\}
$$

being $\widetilde{F}(\phi)$ the corner function given by (3.2) and (3.4). The factor 2 in (3.6) is due to the fact that the two opposite wedges provide the same contribution.

A critical value $\phi_{c}$ for the common opening angle occurs when the two functions compared in (3.6) takes the same value. From the arguments of the $\widetilde{F}$ 's in (3.6), it is straightforward to find that $\phi_{c}=\pi / 2$.

In figure 5 we show two triangulations obtained with Surface Evolver which approximate the corresponding minimal surface $\hat{\gamma}_{A}$ in the two cases of $\phi<\phi_{c}$ (top panel) and $\phi>\phi_{c}$ (bottom panel). The crucial difference between them can be appreciated by focussing around the common tip $W$. Indeed, when $\phi<\phi_{c}$ the points of $\hat{\gamma}_{A}$ close to the tip have coordinates $(x, y) \in A$ and $\hat{\gamma}_{A}$ is made by the union of two minimal surfaces like the one in figure 3 which have the same tip. Instead, when $\phi>\phi_{c}$ the points of $\hat{\gamma}_{A}$ close to the tip have coordinates $(x, y) \notin A$. This leads to the expression (3.6) for the coefficient of the logarithmic divergence in the expansion of $\mathcal{A}\left[\hat{\gamma}_{\varepsilon}\right]$. The minimal surface $\hat{\gamma}_{A}$ is symmetric w.r.t. two half planes orthogonal to the $z=0$ plane whose boundaries are the two straight lines which characterise the symmetry of $A$. In figure 5 the symmetry w.r.t. one of these two half planes is highlighted by the fact that the triangulation is shown only for half of the surface, while the remaining half surface is shaded. This choice makes evident the curve given by the intersection between this half plane and $\hat{\gamma}_{A}$ when $\phi>\phi_{c}$.

In figure 6 we show the results of our numerical analysis for this kind of symmetric regions. The points labeled by red triangles are obtained from triangulated surfaces like the one in the top panel of figure 5, while the points labeled by black circles correspond to triangulated surfaces like the one in the bottom panel of the same figure. The solid blue curve in figure 6 is obtained from the analytic expression (3.6). The agreement of our numerical results with the expected analytic curve is very good. This strongly encourages us to apply this numerical method to study more complicated configurations.

Another class of symmetric configurations is made by double drop regions $A$ which are symmetric with respect to a straight line passing through the vertex $W$. There are two possibilities: either the intersection between this straight line and $A$ is only the common tip (in this case $\phi_{1}=\phi_{2} \equiv \phi$ ) or such intersection is given by a finite segment belonging to $A$ (in this case $\left.\varphi_{1}=\varphi_{2} \equiv \varphi\right)$. In both these cases a constraint reduces the number of independent opening angles to two. Focussing on the coefficient of the logarithmic divergence, one can consider the limit of infinite wedges and employ the property $S_{A}=S_{B}$ of the pure states in this regime. This leads to conclude that these two options are equivalent and that the corresponding corner functions become the same because the property $S_{A}=S_{B}$ allows to 


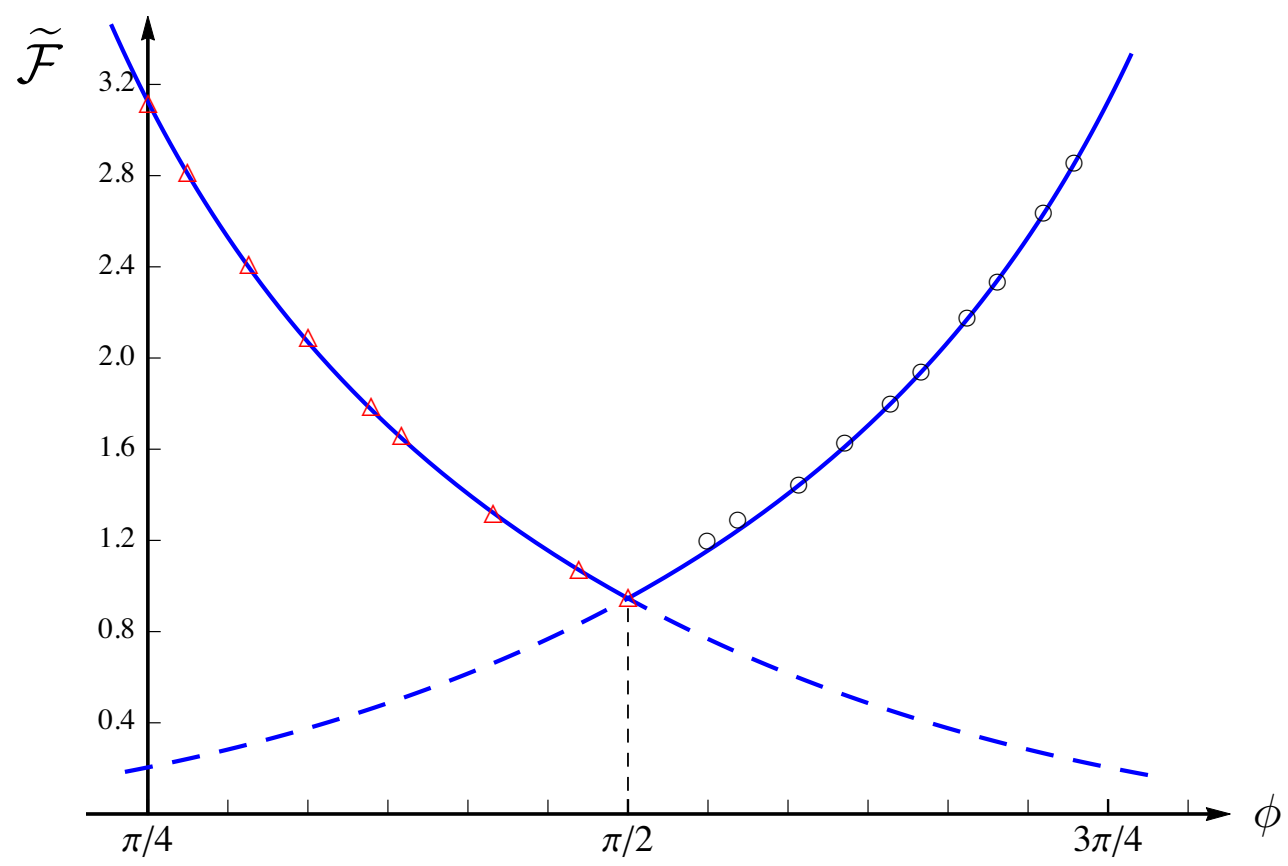

Figure 6. Corner function for a vertex with four edges in $\mathrm{AdS}_{4} / \mathrm{CFT}_{3}$ in the symmetric case where $\phi_{1}=\phi_{2} \equiv \phi$ and $\varphi_{1}=\varphi_{2}=\pi-\phi$ (see section 3.2). The points labeled by the red triangles come from surfaces like the one in the top panel of figure 5, while the points labeled by the black empty circles are obtained from surfaces like the one in the bottom panel of figure 5. The solid curve corresponds to the analytic expression (3.6).

exchange $\varphi_{j} \leftrightarrow \phi_{j}$. Nonetheless, we find it instructive to discuss both of them separately because they look very different when $A$ is a finite domain.

As for the former class of configurations, by choosing the angles $\vec{\phi}=\left(\phi, \varphi_{1}\right)$ as independent variables, the remaining angle $\varphi_{2}$ is determined by the consistency condition $2 \phi+\varphi_{1}+\varphi_{2}=2 \pi$. The area of the minimal surface anchored to this kind of regions is given by (1.7) where $\widetilde{F}_{\text {tot }}=\widetilde{\mathcal{F}}(\vec{\phi})$ and the corner function reads

$$
\widetilde{\mathcal{F}}(\vec{\phi})=\max \left\{2 \widetilde{F}(\phi), \widetilde{F}\left(\varphi_{1}\right)+\widetilde{F}\left(\varphi_{2}\right)\right\}
$$

where we remind that $\widetilde{F}\left(\varphi_{2}\right)=\widetilde{F}\left(\min \left[\varphi_{2}, 2 \pi-\varphi_{2}\right]\right)$. Also this case has been considered in [62]. When the two expressions occurring in the r.h.s. of (3.7) are equal, a transition occurs. This condition determines a critical value $\varphi_{1, c}=\varphi_{1, c}(\phi)$ in terms of $\phi<\pi$. In figure 7 we show two examples of minimal surfaces anchored to double drop regions which have this kind of symmetry. In particular $\varphi_{1}>\varphi_{1, c}$ in the top panel and $\varphi_{1}<\varphi_{1, c}$ in the bottom panel.

Considering the second class of configurations introduced above, where $\varphi_{1}=\varphi_{2} \equiv \varphi$, we have that $\phi_{1}+\phi_{2}+2 \varphi=2 \pi$ and therefore two angles fix the configurations of the corners in the neighbourhood of the common tip. One can choose e.g. $\vec{\phi}=\left(\phi_{1}, \phi_{2}\right)$. For this kind of double drop domains the coefficient of the logarithmic divergence in the area (1.7) is 

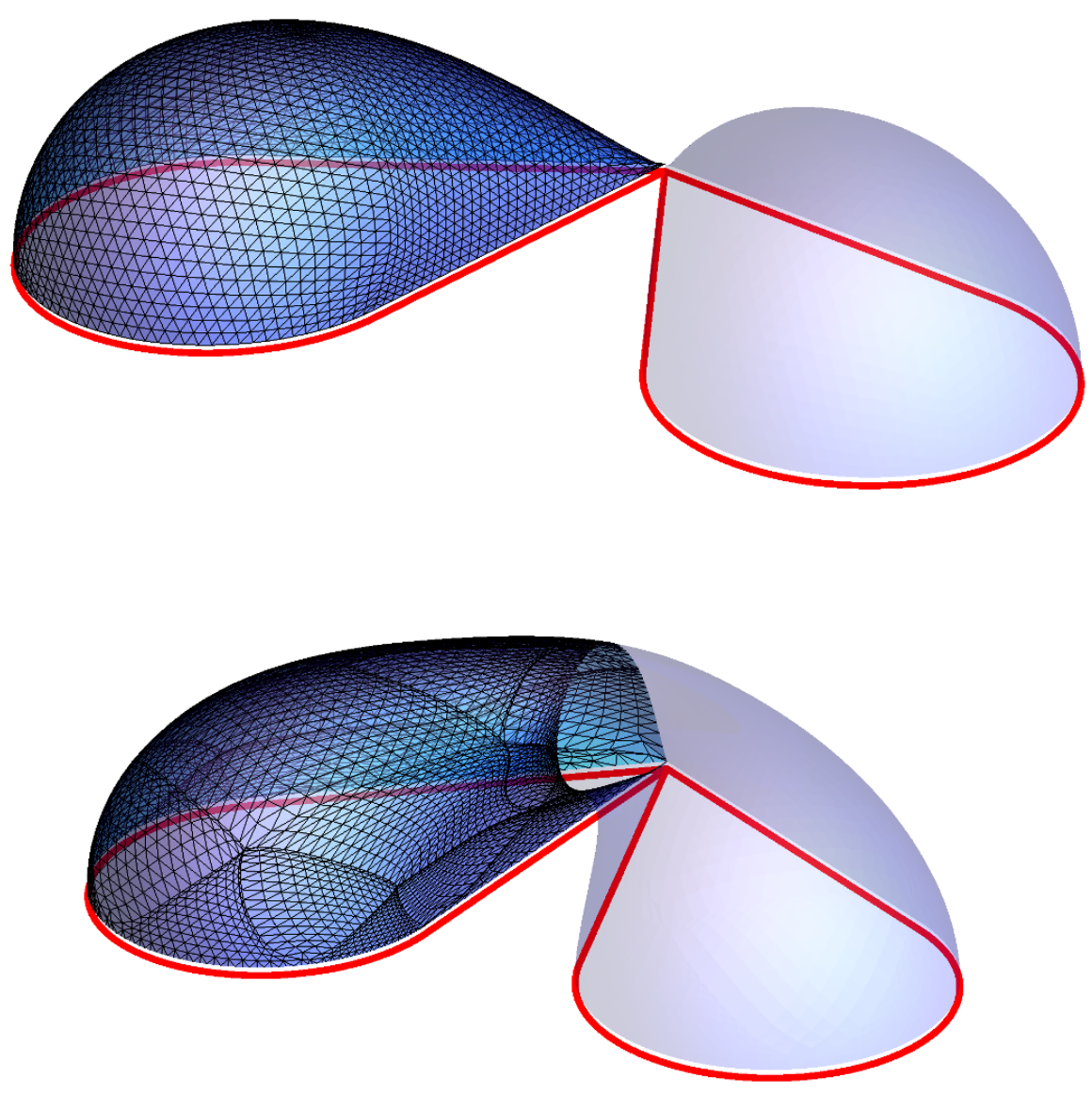

Figure 7. Triangulated surfaces in $\mathbb{H}_{3}$ which approximate the minimal area surfaces $\hat{\gamma}_{A}$ corresponding to two different double drop regions $A$ which are symmetric w.r.t. a straight line passing through the vertex. For these domains $\phi_{1}=\phi_{2} \equiv \phi$ (see section 3.2). The boundary $\partial A$ (red curve) belongs to the $z=0$ plane and the UV cutoff is $\varepsilon=0.03$. Top: $L=1.5$ with $\phi=0.9$ and $\varphi_{1}=0.671$. Bottom: $L=1.5$ with $\phi=0.8$ and $\varphi_{1}=0.378$.

$\widetilde{F}_{\text {tot }}=\widetilde{\mathcal{F}}(\vec{\phi})$ with

$$
\widetilde{\mathcal{F}}(\vec{\phi})=\max \left\{\widetilde{F}\left(\phi_{1}\right)+\widetilde{F}\left(\phi_{2}\right), 2 \widetilde{F}(\varphi)\right\}
$$

As expected, also in this case two local solutions for the minimal surface exist and the global minimum provides the holographic entanglement entropy. The transition between the two kinds of solutions occurs when the two expressions in the r.h.s. of (3.8) are equal and this corresponds to a critical value for $\phi_{1, c}=\phi_{1, c}(\varphi)$. Notice that (3.7) and (3.8) exchange if $\phi_{j} \leftrightarrow \varphi_{j}$, as observed above.

For a generic double drop region $A$ we cannot employ symmetry arguments. Only the constraint $\phi_{1}+\phi_{2}+\varphi_{1}+\varphi_{2}=2 \pi$ holds; therefore the configuration of corners at $W$ is determined by three independent angles, which are e.g. $\vec{\phi}=\left(\phi_{1}, \varphi_{1}, \phi_{2}\right)$. The expansion of the area of the corresponding $\hat{\gamma}_{\varepsilon}$ is $(1.7)$ with $\widetilde{F}_{\text {tot }}=\widetilde{\mathcal{F}}(\vec{\phi})$, with the corner function given 
by

$$
\widetilde{\mathcal{F}}(\vec{\phi})=\max \left\{\widetilde{F}\left(\phi_{1}\right)+\widetilde{F}\left(\phi_{2}\right), \widetilde{F}\left(\varphi_{1}\right)+\widetilde{F}\left(\varphi_{2}\right)\right\}
$$

The transition occurs when the two expressions in the r.h.s. of (3.9) are equal. This condition provides a critical surface in the parameter space described by $\left(\phi_{1}, \varphi_{1}, \phi_{2}\right)$ with $\phi_{1} \leqslant \phi_{2}$.

\section{Holographic entanglement entropy in $\mathrm{AdS}_{4} / \mathrm{BCFT}_{3}$}

In this section we briefly review the AdS/BCFT construction introduced in [63] and further expanded in $[64,65]$. We mainly focus on the computation of the holographic entanglement entropy in the simplest setup where the boundary of the BCFT is a flat hyperplane.

Given a $\mathrm{BCFT}_{d+1}$ in $d+1$ spacetime dimensions, the dual gravitational background proposed in [63] is an asymptotically $\operatorname{AdS}_{d+2}$ spacetime $\mathcal{M}$ restricted by the occurrence of a $d+1$ dimensional hypersurface $\widetilde{\mathcal{Q}}$ whose boundary coincides with the boundary of the $\mathrm{BCFT}_{d+1}$. We consider the simplified setup where the gravitational action reads [63, 64]

$$
\mathcal{I}=\frac{1}{16 \pi G_{\mathrm{N}}} \int_{\mathcal{M}} \sqrt{-g}(R-2 \Lambda)+\frac{1}{8 \pi G_{\mathrm{N}}} \int_{\widetilde{\mathcal{Q}}} \sqrt{-h}(K-T)
$$

being $\Lambda=-\frac{d(d+1)}{2 L_{\mathrm{AdS}}^{2}}$ the negative cosmological constant, $h_{a b}$ the induced metric on $\widetilde{\mathcal{Q}}$ and $K=h^{a b} K_{a b}$ the trace of the extrinsic curvature $K_{a b}$ of $\widetilde{\mathcal{Q}}$. In our analysis the constant $T$ is a real parameter characterising the hypersurface $\widetilde{\mathcal{Q}}$. We have omitted the boundary term due to the fact that the boundary of the gravitational spacetime is non smooth $[78,79]$ along the boundary of the $\mathrm{BCFT}_{d+1}$ and also the boundary terms introduced by the holographic renormalisation procedure [80-86] because they are not relevant in our analysis.

In the AdS/BCFT setup of [63], the Neumann boundary conditions $K_{a b}=(K-T) h_{a b}$ have been proposed to determine the boundary $\widetilde{\mathcal{Q}}$. Instead, in [72-74] it has been suggested that a consistent AdS/BCFT setup can be defined also by considering the less restrictive boundary condition $K=\frac{d+1}{d} T$ to find $\widetilde{\mathcal{Q}}$, obtained by taking the trace of the above Neumann boundary conditions. When the boundary of the $\mathrm{BCFT}_{d+1}$ is a flat $d$ dimensional hyperplane, these two prescriptions provide the same $\widetilde{\mathcal{Q}} \equiv \mathcal{Q}$.

In this manuscript we focus on the simplest case of a $\mathrm{BCFT}_{d+1}$ in its ground state whose boundary is a flat $d$ dimensional hyperplane. Hence, we find it convenient to introduce Cartesian coordinates $(t, x, \vec{y})$ in the $d+1$ dimensional Minkowski spacetime such that the $\mathrm{BCFT}_{d+1}$ is defined in $x \geqslant 0$ and its boundary corresponds to $x=0$. In $[63,64]$ it has been discussed that the gravitational spacetime $\mathcal{M}$ in the bulk dual to the ground state is $\mathrm{AdS}_{d+2}$, whose metric in Poincaré coordinates reads

$$
d s^{2}=\frac{L_{\mathrm{AdS}}^{2}}{z^{2}}\left(-d t^{2}+d z^{2}+d x^{2}+d \vec{y}^{2}\right) \quad z>0
$$

where $d \vec{y}^{2}$ is the metric of $\mathbb{R}^{d-1}$, restricted by the half hyperplane $\mathcal{Q}$ given by ${ }^{1}$

$$
\mathcal{Q}: \quad x=-(\cot \alpha) z \quad \alpha \in(0, \pi)
$$

\footnotetext{
${ }^{1}$ Comparing our notation with the one adopted in [63, 64], we have $\tan \alpha=1 / \sinh \left(\rho_{*} / L_{\mathrm{AdS}}\right)$, being $\rho_{*} \in \mathbb{R}$.
} 
whose boundary at $z=0$ coincides with the boundary of the $\mathrm{BCFT}_{d+1}$, which is $x=0$. The slope $\alpha \in(0, \pi)$ of the half hyperplane $\mathcal{Q}$ is related to the parameter $T$ in the gravitational action (4.1) as $T=\left(d / L_{\mathrm{AdS}}\right) \cos \alpha$.

In our analysis we mainly focus on a $\mathrm{BCFT}_{3}$ defined for $x \geqslant 0$. Hence, a $t=$ const slice of the gravitational bulk is (3.1) constrained by the following condition

$$
x \geqslant-(\cot \alpha) z
$$

which guarantees that the half plane defined by $z=0$ and $x \geqslant 0$ belongs to the boundary of the bulk spacetime.

Given this simple $\mathrm{AdS}_{4} / \mathrm{BCFT}_{3}$ setup, in this manuscript we are interested in the holographic entanglement entropy of some spatial simply connected regions $A$ defined in the spatial half plane $\{(x, y) \mid x \geqslant 0\}$. We compute the holographic entanglement entropy by adapting the prescription (1.6) in the most natural way. Given a region $A$ in the $t=$ const section of the $\mathrm{BCFT}_{3}$, let us split its boundary $\partial A$ as the union of $\partial A_{\mathrm{bdy}}$ (see below (1.4)) and its complementary curve $\partial A \backslash \partial A_{\mathrm{bdy}}$, which corresponds to the entangling curve. In the bulk (3.1) restricted by (4.4), the holographic entanglement entropy is determined by the two dimensional minimal area surface $\hat{\gamma}_{A}$ anchored to the entangling curve.

The latter condition becomes relevant whenever $\partial A_{\mathrm{bdy}}$ contains one dimensional curves. When $\partial A_{\text {bdy }}$ is either empty or made by isolated points, the minimal surface $\hat{\gamma}_{A}$ anchored to the entire $\partial A$ must be considered. It is straightforward to find domains $A$ such that $\partial A_{\text {bdy }}$ contains both one dimensional lines and isolated points. For instance, for the domain $A$ in the right panel of figure 1 the set $\partial A_{\mathrm{bdy}}$ is made by the segment $\overline{P_{1} P_{2}}$ and the isolated point $Q_{1}$.

We find it worth remarking that for some domains $\hat{\gamma}_{A} \cap \mathcal{Q}$ is a non trivial curve. in these cases, since we do not impose any restriction on the intersection between $\hat{\gamma}_{A}$ and $\mathcal{Q}$, it is not difficult to show that $\hat{\gamma}_{A}$ intersects $\mathcal{Q}$ orthogonally along the curve $\hat{\gamma}_{A} \cap \mathcal{Q}$.

Since the minimal surface $\hat{\gamma}_{A}$ constructed in this way reaches the half plane $z=0$, its area is infinite. Thus, the holographic UV cutoff $\varepsilon$ must be introduced and the part of $\hat{\gamma}_{A}$ given by $\hat{\gamma}_{\varepsilon}=\hat{\gamma}_{A} \cap\{z \geqslant \varepsilon\}$ must be considered. Indeed, the holographic entanglement entropy is obtained from the area of $\hat{\gamma}_{\varepsilon}$ as follows

$$
S_{A}=\frac{\mathcal{A}\left[\hat{\gamma}_{\varepsilon}\right]}{4 G_{\mathrm{N}}}
$$

where $G_{\mathrm{N}}$ is the gravitational Newton constant corresponding to four dimensional spacetimes.

The generalisation of (4.5) to a generic boundary and to a generic number of spacetime dimensions is straightforward and the holographic entanglement entropy computed in this way gives $S_{A}=S_{B}$ for pure states. Furthermore, the argument of [58] can be adapted to show that this prescription for the holographic entanglement entropy satisfies the strong subadditivity.

Focussing on the case of $\mathrm{AdS}_{4} / \mathrm{BCFT}_{3}$, we find it worth anticipating that for the domains $A$ in the $z=0$ half plane considered in the following, we find that the corresponding minimal surfaces $\hat{\gamma}_{A}$ are part of auxiliary minimal surfaces $\hat{\gamma}_{A \text {,aux }} \subset \mathbb{H}_{3}$ anchored to the 
boundary of suitable auxiliary domains $A_{\text {aux }} \subset \mathbb{R}^{2}=\partial \mathbb{H}_{3}$. In particular $\hat{\gamma}_{A}$ is the part of $\hat{\gamma}_{A \text {,aux }}$ identified by the constraint (4.4). We remark that $A \subsetneq A_{\text {aux }}$ for the spatial domains, $\hat{\gamma}_{A} \subsetneq \hat{\gamma}_{A \text {,aux }}$ for the minimal surfaces and $\left(\partial A \backslash \partial A_{\text {bdy }}\right) \subsetneq \partial A_{\text {aux }}$ for the entangling curves.

As anticipated in section 1, within the $\mathrm{AdS}_{4} / \mathrm{BCFT}_{3}$ setup described above, we are mainly interested in regions $A$ such that the expansion of the corresponding $\mathcal{A}\left[\hat{\gamma}_{\varepsilon}\right]$ as $\varepsilon \rightarrow 0^{+}$ is given by (1.9) (an example is depicted in the right panel of figure 1) because interesting pieces of information could be encoded in the corner functions $F_{\alpha}(\gamma)$ and $\mathcal{F}_{\alpha}(\omega, \gamma)$. In this holographic context $\alpha$ has a geometrical meaning because it provides the slope of $\mathcal{Q}$ in (4.3). Let us remark that understanding the possible holographic relation between the angle $\alpha$ and the conformally invariant boundary conditions of the $\mathrm{BCFT}_{3}$ in the boundary is still an interesting question that deserves further analysis.

In the following we provide analytic expressions for the corner functions $F_{\alpha}(\gamma)$ and $\mathcal{F}_{\alpha}(\omega, \gamma)$. These functions are checked against numerical results obtained through an independent numerical analysis based on Surface Evolver. Our confidence in this tool relies on the very good results obtained in section 3 for the corner functions in $\mathrm{AdS}_{4} / \mathrm{CFT}_{3}$. In the appendix A we briefly discuss some peculiar features which distinguish our numerical analysis from the one performed in [60,61], where Surface Evolver has been employed to study the shape dependence of the holographic entanglement entropy in $\mathrm{AdS}_{4} / \mathrm{CFT}_{3}$.

In [75] the interesting possibility of including also the length of the curve $\hat{\gamma}_{A} \cap \mathcal{Q}$ in the definition of the holographic entanglement entropy in $\mathrm{AdS}_{4} / \mathrm{BCFT}_{3}$ has been explored. This proposal is discussed in the appendix E.

\section{The half disk and the infinite strip}

In this section we compute analytically the holographic entanglement entropy of two regions which are highly symmetric: the half disk $A$ centered on the boundary (i.e. such that its diameter belonging to $\partial A$ lies on the boundary at $x=0$ ) and an infinite strip parallel to the boundary, either adjacent to it or at a finite distance from it.

\subsection{Half disk centered on the boundary}

Let us consider the half disk $A$ of radius $R$ whose center is located on the boundary of the $\mathrm{BCFT}_{3}$. In the Cartesian coordinates introduced above, where the boundary of the $z=0$ half plane is $x=0$, the translation invariance along the $y$ direction allows to choose the center of the half disk as the origin of the coordinates system. Thus, $A=\{(x, y) \in$ $\left.\mathbb{R}^{2} \mid x^{2}+y^{2} \leqslant R^{2}, x \geqslant 0\right\}$. In $\mathrm{BCFT}_{3}$ the entanglement entropy of this domain has been studied in [55], by using the method of [87].

In our $\mathrm{AdS}_{4} / \mathrm{BCFT}_{3}$ setup the constraint (4.4) due to the occurrence of the half plane $\mathcal{Q}$ must be taken into account. The key observation is that the hemisphere $x^{2}+y^{2}+z^{2}=R^{2}$ in $\mathbb{H}_{3}$ intersects orthogonally the half plane $\mathcal{Q}$ along an arc of circumference of radius $R$ centered in the origin with opening angle equal to $\pi$. It is well known that this hemisphere is the minimal area surface anchored to the circular curve $x^{2}+y^{2}=R^{2}$ in the $z=0$ plane $[5,6,26,88]$. Thus, the minimal surface $\hat{\gamma}_{A}$ corresponding to the half disk $A$ in presence of the brane $\mathcal{Q}$ is part of the minimal area surface $\hat{\gamma}_{A \text {,aux }}=\left\{(x, y, z) \in \mathbb{H}_{3} \mid x^{2}+\right.$ 

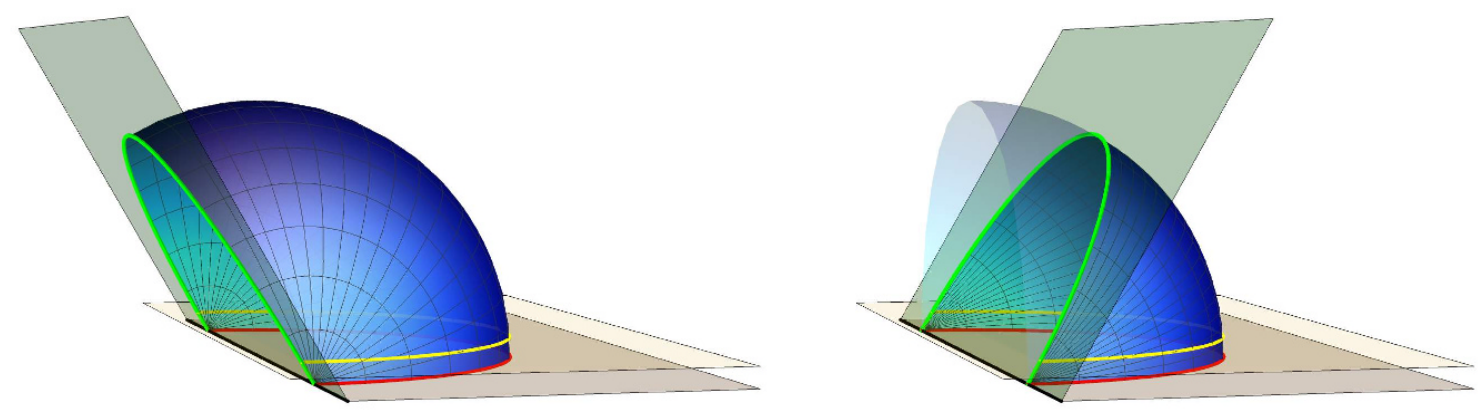

Figure 8. Minimal surfaces $\hat{\gamma}_{A}$ corresponding to the half disk centered on the boundary. The green half plane is $\mathcal{Q}$ in (4.3), while the grey half plane is $z=0$. In the left panel $\alpha<\pi / 2$, while in the right panel $\alpha>\pi / 2$. The green curve is $\hat{\gamma}_{A} \cap \mathcal{Q}$ and the red curve is the entangling curve $\partial A \cap \partial B$, whose length enters in the area law term of (5.1). The yellow half plane is defined by $z=\varepsilon$ and the yellow curve corresponds to its intersection with $\hat{\gamma}_{A}$.

$\left.y^{2}+z^{2}=R^{2}\right\}$ anchored to the boundary of the auxiliary domain $A_{\text {aux }} \subset \mathbb{R}^{2}=\partial \mathbb{H}_{3}$ given by a disk of radius $R$ which includes $A$ as a proper subset. In particular $\hat{\gamma}_{A}$ is the part of $\hat{\gamma}_{A \text {,aux }}$ identified by the constraint (4.4).

In figure 8 we show $\hat{\gamma}_{A}$ for a case having $\alpha<\pi / 2$ in the left panel and for a case with $\alpha>\pi / 2$ in the right panel. Notice that the boundary of $\hat{\gamma}_{A}$ is a continuous curve made by two arcs whose opening angles are equal to $\pi$ : the arc in the $z=0$ half plane defined by $\left\{(x, y) \mid x^{2}+y^{2}=R^{2}, x \geqslant 0\right\}$ and the arc given by $\partial \hat{\gamma}_{\mathcal{Q}} \equiv \hat{\gamma}_{A} \cap \mathcal{Q}$.

Since $\hat{\gamma}_{A}$ reaches the boundary at $z=0$, its area is infinite; therefore we have to introduce the cutoff $\varepsilon>0$ and consider the area of the restricted surface $\hat{\gamma}_{\varepsilon}=\hat{\gamma}_{A} \cap\{z \geqslant \varepsilon\}$ as $\varepsilon \rightarrow 0^{+}$. The details of this computation have been reported in the appendix B. For a given $\alpha \in(0, \pi)$ we find

$$
\mathcal{A}\left[\hat{\gamma}_{\varepsilon}\right]=L_{\text {AdS }}^{2}\left(\frac{\pi R}{\varepsilon}+2(\cot \alpha) \log (R / \varepsilon)+O(1)\right)
$$

This expression is a special case of (1.9) corresponding to $P_{A, B}=\pi R$ and $F_{\alpha, \text { tot }}=2 F_{\alpha}(\pi / 2)$. Thus, we have

$$
F_{\alpha}(\pi / 2)=-\cot \alpha
$$

As consistency check, we observe that $F_{\pi / 2}(\pi / 2)=0$. This is expected because (5.1) for $\alpha=\pi / 2$ gives half of the area of the hemisphere $x^{2}+y^{2}+z^{2}=R^{2}$ restricted to $z \geqslant \varepsilon$ in $\mathbb{H}_{3}$. Furthermore, by increasing the slope $\alpha$ of $\mathcal{Q}$ while $A$ is kept fixed, the area $\mathcal{A}\left[\hat{\gamma}_{\varepsilon}\right]$ in (5.1) decreases because of the coefficient of the logarithmic divergence, as expected.

The result (5.2) can be obtained also by considering a bipartition whose entangling curve is a half straight line orthogonal to the boundary [75].

\subsection{Infinite strip adjacent to the boundary}

A simple domain which plays an important role in our analysis is the infinite strip of finite width $\ell$ adjacent to the boundary, namely such that one of its two edges coincides with the boundary $x=0$. This region has been considered also in [73]. In the following 
we present only the main results about the holographic entanglement entropy of this region in $\mathrm{AdS}_{4} / \mathrm{BCFT}_{3}$. Their detailed derivation in $\mathrm{AdS}_{d+2} / \mathrm{BCFT}_{d+1}$ is reported in the appendix $\mathrm{C}$.

Considering the rectangular domain $A=\left\{(x, y) \in \mathbb{R}^{2} \mid 0 \leqslant x \leqslant \ell, 0 \leqslant y \leqslant L_{\|}\right\}$, the infinite strip adjacent to the boundary is obtained by taking $L_{\|} \gg \ell \gg \varepsilon$. These assumptions allow to assume the invariance under translations in the $y$ direction and this symmetry drastically simplifies the problem of finding the minimal surface $\hat{\gamma}_{A}$ and its area because $\hat{\gamma}_{A}$ is completely characterised by its profile $z=z(x)$ obtained through a section at $y=$ const.

The minimal area surface $\hat{\gamma}_{A}$ intersects the $z=0$ half plane orthogonally along the line $x=\ell$ and this leads to the linear divergence $L_{\|} / \varepsilon$ (area law term) in its area. Let us stress that the logarithmic divergence does not occur in this case.

When $\alpha \leqslant \pi / 2$, two surfaces $\hat{\gamma}_{A}^{\text {dis }}$ and $\hat{\gamma}_{A}^{\text {con }}$ are local extrema of the area functional and the minimal surface $\hat{\gamma}_{A}$ is given by the global minimum. In particular, $\hat{\gamma}_{A}^{\text {dis }}$ is the half plane given by $x=\ell$, therefore it remains orthogonal to the $z=0$ plane and it does not intersect $\mathcal{Q}$ at a finite value of $z$, while $\hat{\gamma}_{A}^{\text {con }}$ bends in the bulk towards the half plane $\mathcal{Q}$ until it intersects it orthogonally at a finite value $z_{*}$ of the coordinate $z$. It is straightforward to observe that the solution $\hat{\gamma}_{A}^{\text {dis }}$ does not exist for $\alpha>\pi / 2$.

The surface $\hat{\gamma}_{A}^{\text {con }}$ can be also viewed as the part identified by the constraint (4.4) of the auxiliary minimal surface $\hat{\gamma}_{A \text {,aux }} \subset \mathbb{H}_{3}$ anchored to the auxiliary infinite strip $A_{\text {aux }} \subset \mathbb{R}^{2}$ which includes $A$ and has one of its edges at $x=\ell$. In the appendix $\mathrm{C}$ the width of $A_{\text {aux }}$ has been computed (see (C.16) specialised to $d=2$ ).

Focussing on a section at $y=$ const of $\hat{\gamma}_{A}^{\text {con }}$, which is characterised by the profile $z(x)$, let us denote by $P_{*}=\left(x_{*}, z_{*}\right)$ the intersection between this curve and the half line (4.3) corresponding to $\mathcal{Q}$. In the half plane described by the pair $(z, x)$, we find it convenient to write the curve $z(x)$ of $\hat{\gamma}_{A}$ in a parametric form $P_{\theta}=(x(\theta), z(\theta))$ in terms of the angular variable $\theta \in[0, \pi-\alpha]$. The angular variable $\theta$ corresponds to the angle between the outgoing vector normal to the curve given by $P_{\theta}$ and the $x$ semi-axis with $x \geqslant 0$. The parametric expressions $P_{\theta}$ must satisfy the boundary conditions $P_{0}=(\ell, 0)$ and $P_{\pi-\alpha}=P_{*}$. Since $P_{*}$ lies on $\mathcal{Q}$, we have $x_{*}=-z_{*} \cot \alpha$; therefore we can write its position as $P_{*}=z_{*}(-\cot \alpha, 1)$. In figure 9 we show the profile $z(x)$ corresponding to a given strip adjacent to the boundary for different values of the slope $\alpha$ of $\mathcal{Q}$. Notice that $z_{*}$ is a decreasing function of $\alpha$.

In the appendix $\mathrm{C}$ we find that, for any given slope $\alpha \in(0, \pi)$, the coordinate $z_{*}$ of $P_{*}$ is related to the width $\ell$ of the strip as follows

$$
z_{*}=\frac{\sqrt{\sin \alpha}}{\mathfrak{g}(\alpha)} \ell
$$

where we have introduced

$$
\mathfrak{g}(\alpha) \equiv \mathbb{E}(\pi / 4-\alpha / 2 \mid 2)-\frac{\cos \alpha}{\sqrt{\sin \alpha}}+\frac{\Gamma\left(\frac{3}{4}\right)^{2}}{\sqrt{2 \pi}}
$$

being $\mathbb{E}(\phi \mid m)$ the elliptic integral of the second kind. The expressions (5.3) and (5.4) correspond respectively to (C.10) and (C.11) specialised to $d=2$. In order to enlighten the notation, in the main text we slightly change the notation with respect to the appendix $\mathrm{C}$ 

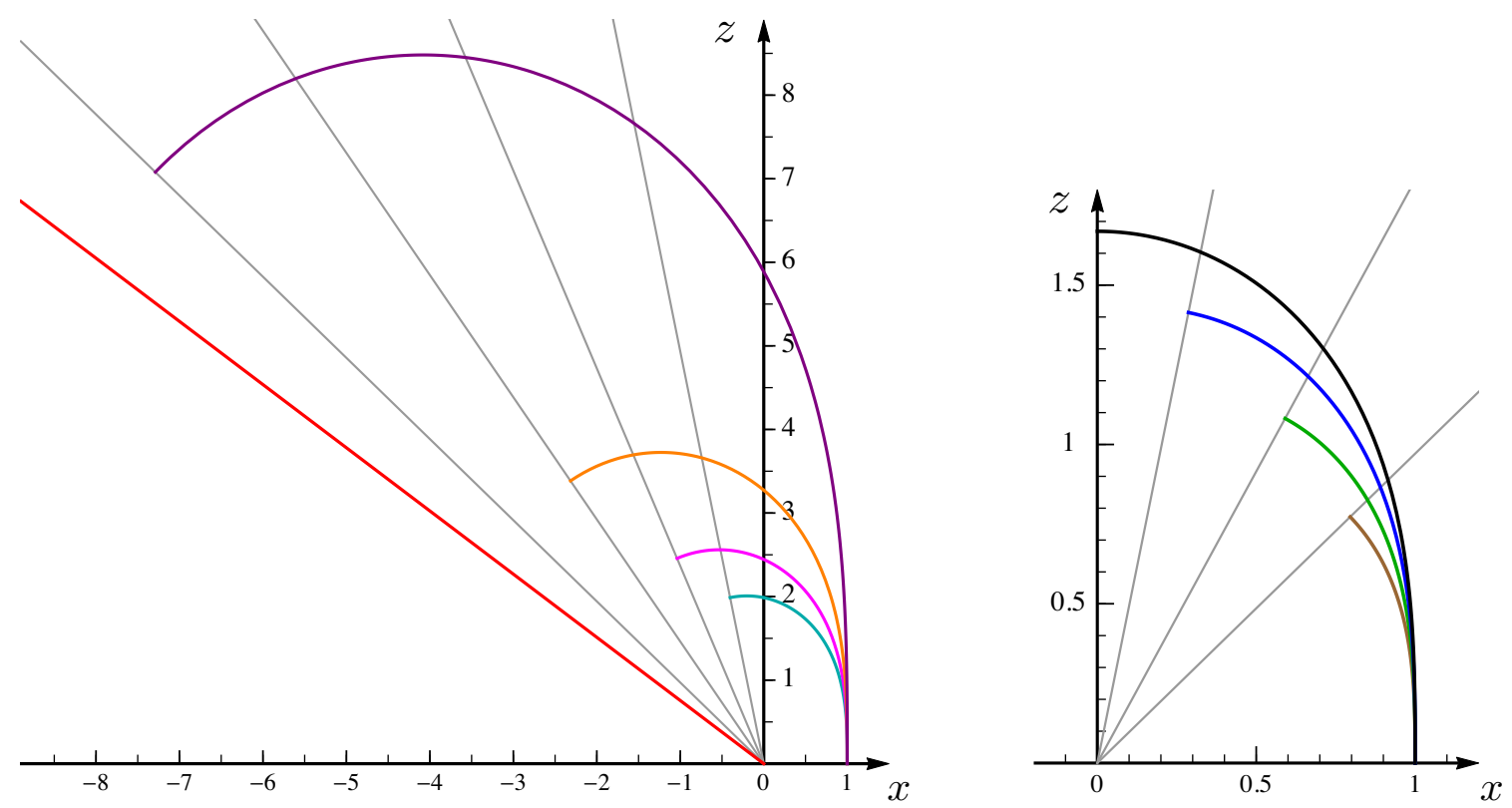

Figure 9. Sections of minimal surfaces $\hat{\gamma}_{A}$ corresponding to an infinite strip adjacent to the boundary whose width is $\ell=1$ for different values of $\alpha>\alpha_{c}$, where $\alpha_{c}$ is given by (5.6). This curves are obtained from (5.7). The grey half lines correspond to the sections of $\mathcal{Q}$ at $y=$ const obtained from (4.3) and the red one is associated to $\alpha=\alpha_{c}$. Each curve intersects orthogonally the corresponding section of $\mathcal{Q}$ at the point $P_{*}$, whose coordinate $z_{*}$ along the $z$ axis is (5.3).

by setting $\mathfrak{g}(\alpha) \equiv \mathfrak{g}_{2}(\alpha)$ (see (C.11)). In figure 10 the function $\mathfrak{g}(\alpha)$ and the ratio $z_{*} / \ell$ are shown in terms of $\alpha \in(0, \pi)$.

As for the function $\mathfrak{g}(\alpha)$ in (5.4), we find $\mathfrak{g}(\alpha)=-1 / \sqrt{\alpha}+O(1)$ when $\alpha \rightarrow 0^{+}$and $\mathfrak{g}(\alpha)=1 / \sqrt{\pi-\alpha}+O(1)$ as $\alpha \rightarrow \pi^{-}$. Moreover $\mathfrak{g}^{\prime}(\alpha)=(\sin \alpha)^{-3 / 2} / 2$ is positive in the whole domain $\alpha \in(0, \pi)$. These observations imply that $\mathfrak{g}(\alpha)$ has a unique zero, namely

$$
\mathfrak{g}\left(\alpha_{c}\right)=0
$$

where we have introduced $\alpha_{c}$ to label the unique solution of this transcendental equation. Solving (5.5) numerically, we find

$$
\alpha_{c} \simeq \frac{\pi}{4.8525821} \simeq 0.647406
$$

Since $z_{*}>0$ in (5.3), the condition (5.5) defines the critical value for the slope $\alpha$ characterising the range of validity of (5.3), which is well defined only for $\alpha \in\left(\alpha_{c}, \pi\right)$. Thus, for $\alpha \leqslant \alpha_{c}$ the solution $\hat{\gamma}_{A}^{\text {con }}$ does not exist and therefore $\hat{\gamma}_{A}=\hat{\gamma}_{A}^{\text {dis }}$. This is confirmed also by the fact that, by taking $\alpha \rightarrow \alpha_{c}^{+}$in (5.3) we have $z_{*} \rightarrow+\infty$. The occurrence of the critical value (5.6) has been observed also in [73].

When $\alpha>\alpha_{c}$, the extremal surface $\hat{\gamma}_{A}^{\text {con }}$ is parametrically described by the following curve

$$
P_{\theta}=(x(\theta), z(\theta))=\frac{\ell}{\mathfrak{g}(\alpha)}\left(\mathbb{E}(\pi / 4-\alpha / 2 \mid 2)-\frac{\cos \alpha}{\sqrt{\sin \alpha}}+\mathbb{E}(\pi / 4-\theta / 2 \mid 2), \sqrt{\sin \theta}\right)
$$




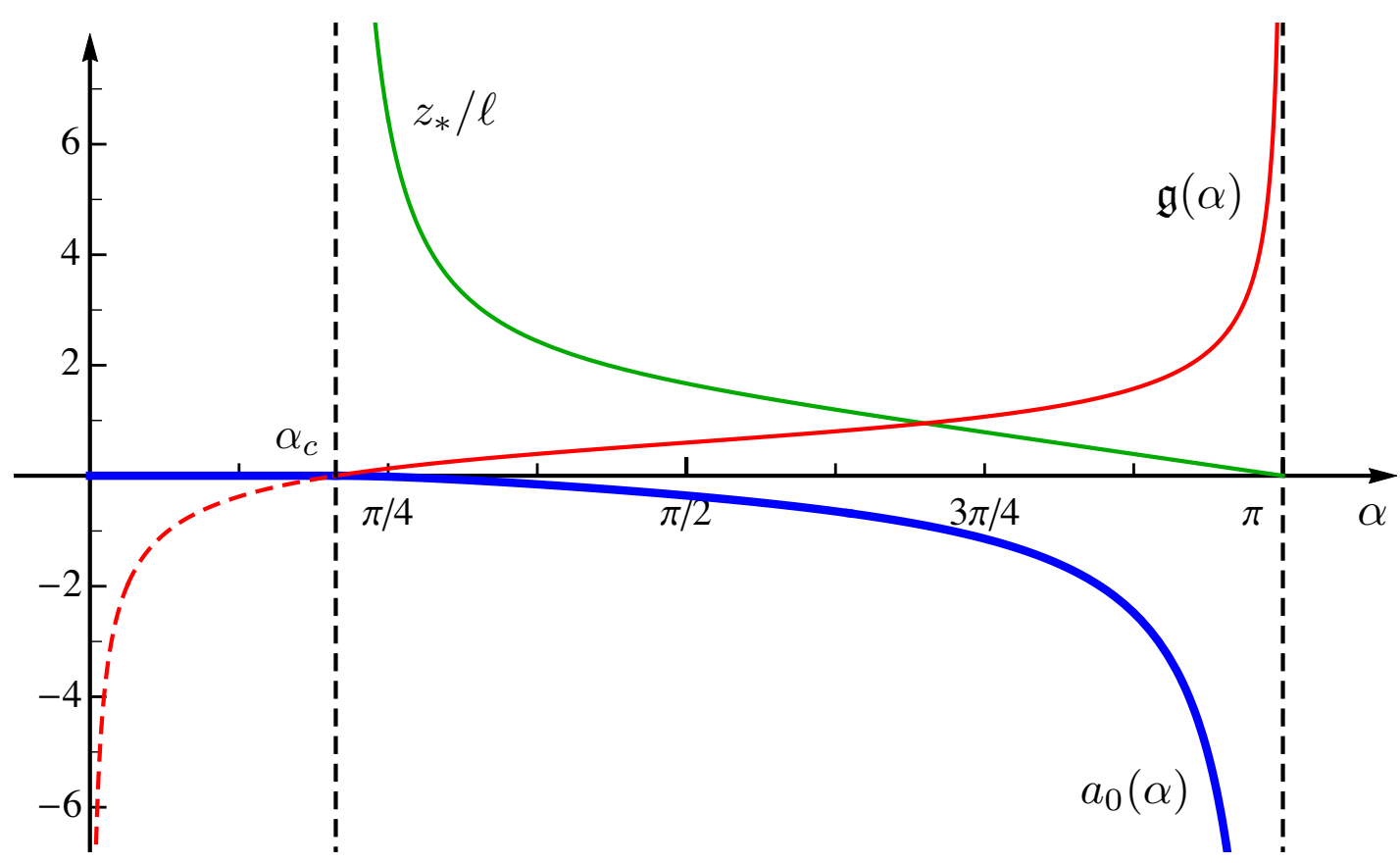

Figure 10. Infinite strip adjacent to the boundary: the red curve is $\mathfrak{g}(\alpha)$ in (5.4), which is positive for $\alpha \geqslant \alpha_{c}$ and negative for $\alpha \leqslant \alpha_{c}$, being $\alpha_{c}$ given by (5.6). The solid green curve corresponds to $z_{*} / \ell$ obtained from (5.3) and it diverges as $\alpha \rightarrow \alpha_{c}^{+}$. The solid blue line is the $O(1)$ term in the expansion (5.9) of the area $\mathcal{A}\left[\hat{\gamma}_{\varepsilon}\right]$.

where the independent angular parameter is $0 \leqslant \theta \leqslant \pi-\alpha$. The profile (5.7) corresponds to (C.14) specialised to $d=2$. It is straightforward to check that (5.7) fulfils the required boundary conditions $P_{0}=(\ell, 0)$ and $P_{\pi-\alpha}=P_{*}=z_{*}(-\cot \alpha, 1)$, with $z_{*}$ given by (5.3). In figure 9 we show the profiles $z(x)$ for $\hat{\gamma}_{A}$ obtained from (5.7) which correspond to the same strip adjacent to the boundary in the $z=0$ half plane ( $\ell=1$ in the figure) and different values of $\alpha$. As for the maximum value $z_{\max }$ reached by the coordinate $z$ along the curve (5.7), we observe that $z_{\max }=z_{*}$ when $\alpha \in[\pi / 2, \pi)$, while $z_{\max }>z_{*}$ for $\alpha \in\left(\alpha_{c}, \pi / 2\right)$.

The expansion for $\varepsilon \rightarrow 0^{+}$of the area of the extremal surface corresponding to the infinite strip adjacent to the boundary and characterised by the curve (5.7) restricted to $z \geqslant \varepsilon$ reads

$$
\mathcal{A}\left[\hat{\gamma}_{\varepsilon}\right]=L_{\text {AdS }}^{2} L_{\|}\left(\frac{1}{\varepsilon}-\frac{\mathfrak{g}(\alpha)^{2}}{\ell}+O\left(\varepsilon^{3}\right)\right) \quad \alpha>\alpha_{c}
$$

This expression is the special case $d=2$ of (C.24). Comparing (5.8) with (1.9), we have that in this case $P_{A, B}=L_{\|}$, the logarithmic divergence does not occur and the $O(1)$ term is negative. The result (5.8) restricted to $\alpha \in(\pi / 2, \pi)$ has been first found in [89]. ${ }^{2}$

An important role in our analysis is played by the extremal surface $\hat{\gamma}_{A}^{\text {dis }}$ given by the vertical half plane at $x=\ell$. By computing its area restricted to $\varepsilon \leqslant z \leqslant z_{\mathrm{IR}}$, being $z_{\mathrm{IR}} \gg \ell$ an infrared cutoff, one easily finds that $\mathcal{A}\left[\hat{\gamma}_{\varepsilon}\right]=L_{\mathrm{AdS}}^{2} L_{\|}\left(1 / \varepsilon-1 / z_{\mathrm{IR}}\right)$. Notice that the $O(1)$ term of this expression vanishes in the limit $z_{\mathrm{IR}} \rightarrow+\infty$. This extremal surface exists only

\footnotetext{
${ }^{2}$ Comparing with the notation of [89], we find that $\kappa_{\text {there }}=-\cot \alpha$.
} 
for $\alpha \leqslant \pi / 2$ because when $\alpha>\pi / 2$ the half plane $\mathcal{Q}$ and the vertical infinite strip $x=\ell$ do not intersect orthogonally.

Summarising, for the minimal area surface $\hat{\gamma}_{A}$ we have that $\hat{\gamma}_{A}=\hat{\gamma}_{A}^{\text {dis }}$ when $\alpha \leqslant \alpha_{c}$ because (5.3) is not well defined. When $\alpha \in\left(\alpha_{c}, \pi / 2\right]$, two extremal surfaces $\hat{\gamma}_{A}^{\text {dis }}$ and $\hat{\gamma}_{A}^{\text {con }}$ compete (the vertical half plane at $x=\ell$ and the surface characterised by (5.7) respectively), while for $\alpha>\pi / 2$ we have $\hat{\gamma}_{A}=\hat{\gamma}_{A}^{\text {con }}$ because $\hat{\gamma}_{A}^{\text {dis }}$ does not exist. As for the regime $\alpha \in\left(\alpha_{c}, \pi / 2\right]$, since the $O(1)$ term in (5.8) is negative while it vanishes for $\hat{\gamma}_{A}^{\text {dis }}$, we conclude that $\hat{\gamma}_{A}=\hat{\gamma}_{A}^{\text {con }}$, given by (5.7).

Combining the above observations, we find that the expansion as $\varepsilon \rightarrow 0^{+}$of the area of the minimal surface $\hat{\gamma}_{A} \cap\{z \geqslant \varepsilon\}$ corresponding to an infinite strip of width $\ell$ adjacent to the boundary for $\alpha \in(0, \pi)$ is

$$
\mathcal{A}\left[\hat{\gamma}_{\varepsilon}\right]=L_{\mathrm{AdS}}^{2} L_{\|}\left(\frac{1}{\varepsilon}+\frac{a_{0}(\alpha)}{\ell}+o(1)\right) \quad a_{0}(\alpha)=\left\{\begin{array}{cc}
-\mathfrak{g}(\alpha)^{2} & \alpha \geqslant \alpha_{c} \\
0 & \alpha \leqslant \alpha_{c}
\end{array}\right.
$$

where $\mathfrak{g}(\alpha)$ has been defined in (5.4) and $\alpha_{c}$ is its unique zero (5.6). The result (5.9) is the special case $d=2$ of the expressions (C.24) and (C.27). Since $\alpha_{c}$ is defined by (5.5), the function $a_{0}(\alpha)$ in (5.9) is continuous and it corresponds to the blue solid curve in figure 10. Let us also observe that $\mathfrak{g}^{\prime}(\alpha)$ is continuous but $\mathfrak{g}^{\prime \prime}(\alpha)$ is not continuous at $\alpha=\alpha_{c}$.

\subsection{Infinite strip parallel to the boundary}

The results for the infinite strip adjacent to the boundary discussed in section 5.2 allow to address also the holographic entanglement entropy of an infinite strip $A$ parallel to the boundary and at finite distance from it. In the appendix D we discuss the analogue case in a $\mathrm{BCFT}_{d+1}$. In the following we report only the results of that analysis for $d=2$.

The configuration of an infinite strip parallel to the boundary is characterised by the width $\ell_{A}$ of the strip and by its distance $d_{A}$ from the boundary. By employing the translation invariance and the results of section 5.2, one realises that $\hat{\gamma}_{A}$ is the global minimum obtained by comparing the area of two possible configurations $\hat{\gamma}_{A}^{\text {dis }}$ and $\hat{\gamma}_{A}^{\text {con }}$. The surface $\hat{\gamma}_{A}^{\text {dis }}$ is disconnected from $\mathcal{Q}$ and it connects the two parallel lines of $\partial A$ through the bulk, while $\hat{\gamma}_{A}^{\text {con }}$ is made by two disjoint surfaces such that each of them connects an edge of $\partial A$ to $\mathcal{Q}$. The two disjoint surfaces occurring in $\hat{\gamma}_{A}^{\text {con }}$ are like the ones described in section 5.2; therefore $\hat{\gamma}_{A}^{\text {con }} \cap \mathcal{Q}$ is made by two parallel lines. The two configurations $\hat{\gamma}_{A}^{\text {dis }}$ and $\hat{\gamma}_{A}^{\text {con }}$ are depicted in figure 11 for a given value of $\alpha$

For an infinite strip $A$ at a finite distance from the boundary, $\hat{\gamma}_{A \text {,aux }}$ is the minimal surface in $\mathbb{H}_{3}$ anchored to $A_{\text {aux }}=A \cup A^{\prime} \subset \mathbb{R}^{2}$, which is the union of two parallel and disjoint infinite strips in $\mathbb{R}^{2}$ [90]. The minimal surface $\hat{\gamma}_{A}$ is the part of $\hat{\gamma}_{A \text {,aux }}$ identified by the constraint (4.4). The width of $A^{\prime}$ and the separation between $A$ and $A^{\prime}$ are given by (D.1) specialised to the case $d=2$.

As for the area of $\hat{\gamma}_{\varepsilon}$, we find

$$
\mathcal{A}\left[\hat{\gamma}_{\varepsilon}\right]=L_{\text {AdS }}^{2} L_{\|}\left(\frac{2}{\varepsilon}+\frac{1}{\ell_{A}} \min \left[h_{2}, a_{0}(\alpha)\left(\frac{1}{\delta_{A}}+\frac{1}{\delta_{A}+1}\right)\right]+o(1)\right) \quad \delta_{A} \equiv \frac{d_{A}}{\ell_{A}}
$$




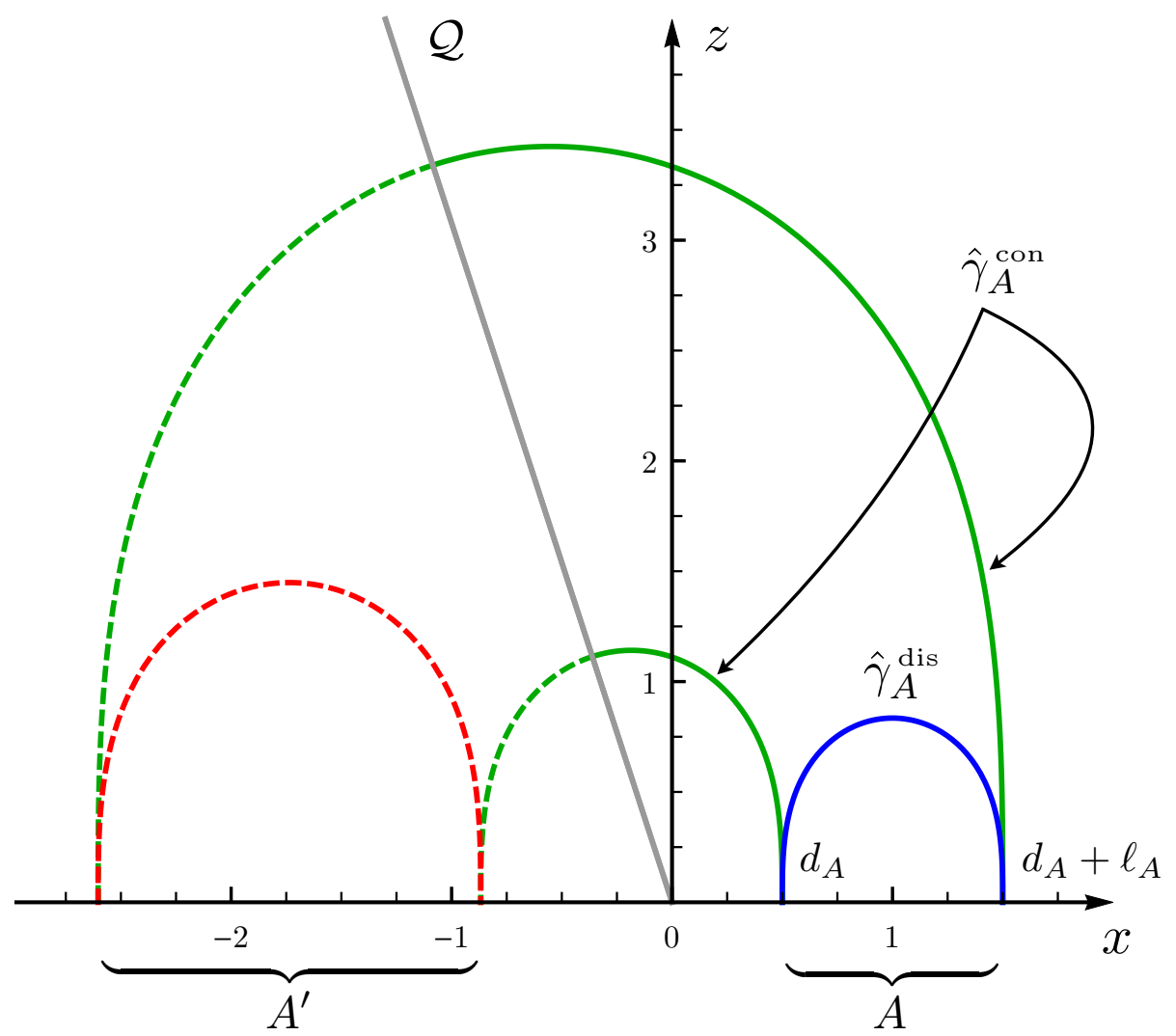

Figure 11. Infinite strip of width $\ell_{A}$ parallel to the boundary at distance $d_{A}$ : section of the surfaces $\hat{\gamma}_{A}^{\text {dis }}$ and $\hat{\gamma}_{A}^{\text {con }}$ (blue and green solid curve respectively) which are local extrema of the area functional. In this plot $\alpha>\alpha_{c}$. The auxiliary domain $A_{\text {aux }}=A \cup A^{\prime}$ in $\mathbb{R}^{2}$ is made by two parallel infinite strips $A$ and $A^{\prime}$. The green dashed curves together with $\hat{\gamma}_{A}^{\text {con }}$ provide $\hat{\gamma}_{A \text {,aux }}$ when $\hat{\gamma}_{A}=\hat{\gamma}_{A}^{\text {con }}$, while the red dashed curve together with $\hat{\gamma}_{A}^{\text {dis }}$ gives $\hat{\gamma}_{A \text {,aux }}$ when $\hat{\gamma}_{A}=\hat{\gamma}_{A}^{\text {dis }}$.

where $a_{0}(\alpha)$ has been introduced in $(5.9)$ and $h_{2} \equiv-4 \pi\left[\Gamma\left(\frac{3}{4}\right) / \Gamma\left(\frac{1}{4}\right)\right]^{2}$ comes from the $O(1)$ term of the holographic entanglement entropy of an infinite strip in $\mathrm{CFT}_{3}[5,6]$. The expression (5.10) corresponds to the special case $d=2$ of (D.2). When $\alpha \leqslant \alpha_{c}$, we have that $\hat{\gamma}_{A}=\hat{\gamma}_{A}^{\text {dis }}$ because $a_{0}(\alpha)=0$ and $h_{2}<0$.

The critical configuration corresponds to the value $\delta_{A}=\delta_{A, c}$ such that the two terms occurring in the minimisation procedure in (5.10) provide the same result. By imposing this condition, one finds an algebraic equation of second order with only one positive root given $b^{3}$

$$
\delta_{A, c}=\frac{1}{2}\left(\sqrt{4\left[a_{0}(\alpha) / h_{2}\right]^{2}+1}+2 a_{0}(\alpha) / h_{2}-1\right)
$$

When $\delta_{A} \leqslant \delta_{A, c}$ the minimal surface is $\hat{\gamma}_{A}=\hat{\gamma}_{A}^{\text {con }}$, while for $\delta_{A} \geqslant \delta_{A, c}$ it is given by $\hat{\gamma}_{A}=\hat{\gamma}_{A}^{\text {dis }}$. The function (5.11) corresponds to the red curve in figure 24 and it is meaningful for $\alpha \geqslant \alpha_{c}$.

\footnotetext{
${ }^{3}$ The formula (5.11) (first presented in [91]) restricted to $\alpha \geqslant \pi / 2$ corresponds to a special case of a result concerning the expectation value of antiparallel Wilson lines in $\mathcal{N}=4 \mathrm{SYM}$ at strong coupling in the presence of a defect, which has appeared later in [92]. In particular, (5.11) can be obtained from eq. (4.15) of [92] by setting $\left.\phi\right|_{\text {there }}=\pi / 2$ and $\left.\chi_{+}\right|_{\text {there }}=\left.\chi_{-}\right|_{\text {there }}=\pi / 2$.
} 


\section{Infinite wedge adjacent to the boundary}

In this section we discuss the main result of this manuscript. In the $\mathrm{AdS}_{4} / \mathrm{BCFT}_{3}$ setup that we are considering, we compute the minimal surface $\hat{\gamma}_{A}$ corresponding to an infinite wedge with opening angle $\gamma \in(0, \pi / 2]$ having one of its edges on the boundary of the $\mathrm{BCFT}_{3}$. By evaluating the area of $\hat{\gamma}_{\varepsilon}$, an analytic expression for the corner function $F_{\alpha}(\gamma)$ occurring in (1.10) is obtained. In the following we report only the main results of our analysis, while the technical details of their derivations are collected in the appendix $\mathrm{F}$.

Let us adopt the polar coordinates $(\rho, \phi)$ given by $x=\rho \sin \phi$ and $y=\rho \cos \phi$, being $(x, y)$ the Cartesian coordinates employed in section 5 for the $t=$ const slice of the $\mathrm{BCFT}_{3}$. In terms of these polar coordinates, we consider the domain $A=\{(\rho, \phi) \mid 0 \leqslant \phi \leqslant \gamma, \rho \leqslant L\}$ with $L \gg \varepsilon$, which is an infinite wedge with one of its two edges on the boundary. Since the wedge is infinite, we can look for the corresponding minimal surface $\hat{\gamma}_{A}$ among the surfaces described by the following ansatz

$$
z=\frac{\rho}{q(\phi)}
$$

where $q(\phi)>0$, as already done in [27] to get the minimal surface in $\mathbb{H}_{3}$ anchored to an infinite wedge in $\mathbb{R}^{2}$.

The minimal surface $\hat{\gamma}_{A}$ can be found as part of an auxiliary minimal surface $\hat{\gamma}_{A \text {,aux }}$ embedded in $\mathbb{H}_{3}$ and anchored to an auxiliary infinite wedge $\hat{\gamma}_{A \text {,aux }}$ containing $A$ and having the same edge $\{(\rho, \phi) \mid \phi=\gamma\}$. The minimal surface $\hat{\gamma}_{A}$ intersects orthogonally the half plane at $z=0$ along the edge $\{(\rho, \phi) \mid \phi=\gamma\}$ of $A$ and the half plane $\mathcal{Q}$ along the half line given by $\phi=\phi_{*}$. As remarked for the previous cases, $\hat{\gamma}_{A}$ is the part of $\hat{\gamma}_{A \text {,aux }}$ identified by the constraint (4.4). For the infinite wedge $A$ that we are considering, $A_{\text {aux }}$ is a suitable infinite wedge in $\mathbb{R}^{2}$ and $\hat{\gamma}_{A \text {,aux }}$ is the corresponding minimal surface found in [27]. In the figure 26 described in the appendix $\mathrm{F}$ the auxiliary wedge $A_{\text {aux }}$ is shown.

Given the half plane $\mathcal{Q}$ described by (4.3), whose slope is $\alpha \in(0, \pi)$, the angle $\phi_{*}$ which identifies the half line $\hat{\gamma}_{A} \cap \mathcal{Q}$ can be defined by introducing the following positive function

$$
s_{*}\left(\alpha, q_{0}\right) \equiv-\eta_{\alpha} \frac{\cot \alpha}{\sqrt{2}}\left\{\frac{\sqrt{1+4(\sin \alpha)^{2}\left(q_{0}^{4}+q_{0}^{2}\right)}-\cos (2 \alpha)}{(\cos \alpha)^{2}+q_{0}^{4}+q_{0}^{2}}\right\}^{\frac{1}{2}} \quad \eta_{\alpha} \equiv-\operatorname{sign}(\cot \alpha)
$$

where $q\left(\phi_{0}\right) \equiv q_{0}>0$ is the value of the function $q(\phi)$ at the angle $\phi=\phi_{0}$ corresponding to the bisector of the auxiliary wedge $A_{\text {aux }}$. We find it convenient to adopt $q_{0}$ as parameter to define various quantities in the following. From (6.2), we find $\phi_{*}$ as

$$
\phi_{*}\left(\alpha, q_{0}\right)=\eta_{\alpha} \arcsin \left[s_{*}\left(\alpha, q_{0}\right)\right]
$$

This result encodes the condition that $\hat{\gamma}_{A}$ intersects $\mathcal{Q}$ orthogonally, as explained in the appendix F.2.

In order to write the analytic expression for the opening angle $\gamma$ of the infinite wedge in terms of the positive parameter $q_{0}$, let us introduce

$$
q_{*}\left(\alpha, q_{0}\right)=\frac{|\cot \alpha|}{s_{*}\left(\alpha, q_{0}\right)}
$$



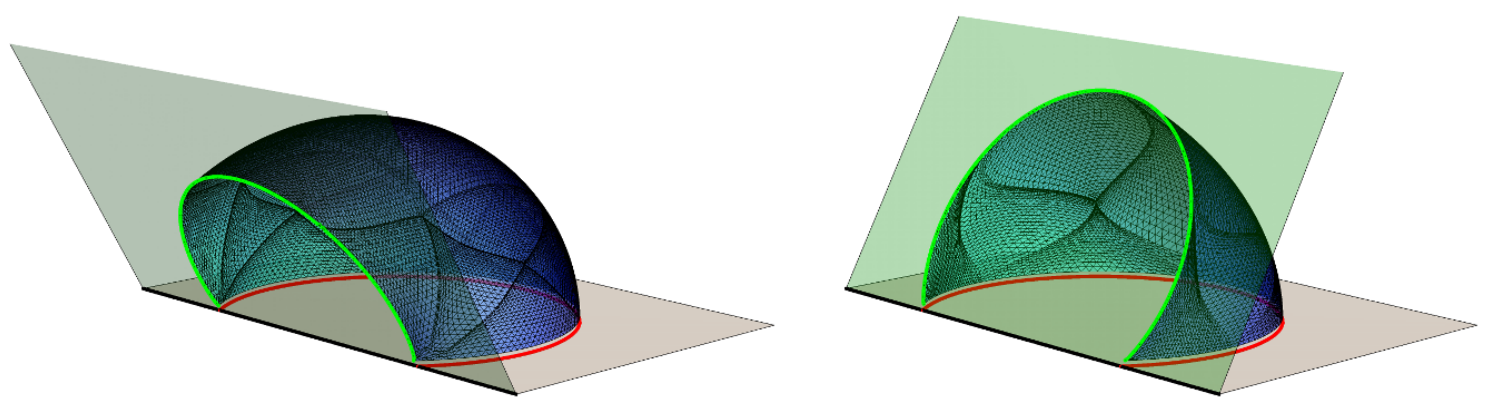

Figure 12. Minimal surfaces $\hat{\gamma}_{A}$ obtained with Surface Evolver corresponding to a region $A$ given by the intersection between the grey half plane at $z=0$ and a disk of radius $R$ whose center has coordinate $x>0$. The entangling curve $\partial A \cap \partial B$ (red line) is an arc of circumference. The green half plane is $\mathcal{Q}$ defined by (4.3) and the green curve corresponds to $\hat{\gamma}_{A} \cap \mathcal{Q}$. In the figure $\varepsilon=0.03$, $R=1$ and the center of the disk has coordinate $x=0.6$. In the left panel $\alpha=\pi / 3$, while in the right panel $\alpha=2 \pi / 3$. The numerical data of the corner function $F_{\alpha}(\gamma)$ corresponding to this kind of domains are labeled by empty circles in figure 14 .

where $s_{*}\left(\alpha, q_{0}\right)>0$ is given by (6.2). For the opening angle $\gamma$ of $A$ we find

$$
\gamma=P_{0}\left(q_{0}\right)+\eta_{\alpha}\left(\arcsin \left[s_{*}\left(\alpha, q_{0}\right)\right]-P\left(q_{*}\left(\alpha, q_{0}\right), q_{0}\right)\right)
$$

where the function $P\left(q, q_{0}\right)$ is defined as

$$
P\left(q, q_{0}\right) \equiv \frac{1}{q_{0}\left(1+q_{0}^{2}\right)}\left\{\left(1+2 q_{0}^{2}\right) \Pi\left(-1 / Q_{0}^{2}, \sigma\left(q, q_{0}\right) \mid-Q_{0}^{2}\right)-q_{0}^{2} \mathbb{F}\left(\sigma\left(q, q_{0}\right) \mid-Q_{0}^{2}\right)\right\}
$$

(in (F.9) we give the integral representation) being $\mathbb{F}(\phi \mid m)$ and $\Pi(n, \phi \mid m)$ the incomplete elliptic integrals of the first and third kind respectively, with

$$
\sigma\left(q, q_{0}\right) \equiv \arctan \sqrt{\frac{q^{2}-q_{0}^{2}}{1+2 q_{0}^{2}}} \quad Q_{0}^{2} \equiv \frac{q_{0}^{2}}{1+q_{0}^{2}} \in(0,1)
$$

The function $P_{0}\left(q_{0}\right)$ in (6.5) is the limit $P\left(q, q_{0}\right) \rightarrow P_{0}\left(q_{0}\right)$ as $q \rightarrow+\infty$. The explicit expression of $P_{0}\left(q_{0}\right)$ in terms of the complete elliptic integrals has been written in (3.4), but we find it convenient to provide here also an equivalent form coming directly from (6.6), namely

$$
P_{0}\left(q_{0}\right)=\frac{1}{q_{0}\left(1+q_{0}^{2}\right)}\left\{\left(1+2 q_{0}^{2}\right) \Pi\left(-1 / Q_{0}^{2},-Q_{0}^{2}\right)-q_{0}^{2} \mathbb{K}\left(-Q_{0}^{2}\right)\right\}
$$

being $\mathbb{K}(m)$ and $\Pi(n \mid m)$ the complete elliptic integrals of the first and third kind respectively.

As for the holographic entanglement (4.5) of the infinite wedge $A$ adjacent to the boundary, since $\hat{\gamma}_{A}$ reaches the boundary $z=0$, its area is infinite; therefore we have to consider its restriction $\hat{\gamma}_{\varepsilon}=\hat{\gamma}_{A} \cap\{z \geqslant \varepsilon\}$ and take the limit $\varepsilon \rightarrow 0^{+}$, as required by the prescription (4.5).

We find that the expansion of the area $\mathcal{A}\left[\hat{\gamma}_{\varepsilon}\right]$ of $\hat{\gamma}_{\varepsilon}$ as $\varepsilon \rightarrow 0$ reads

$$
\mathcal{A}\left[\hat{\gamma}_{\varepsilon}\right]=L_{\text {AdS }}^{2}\left(\frac{L}{\varepsilon}-F_{\alpha}(\gamma) \log (L / \varepsilon)+O(1)\right)
$$



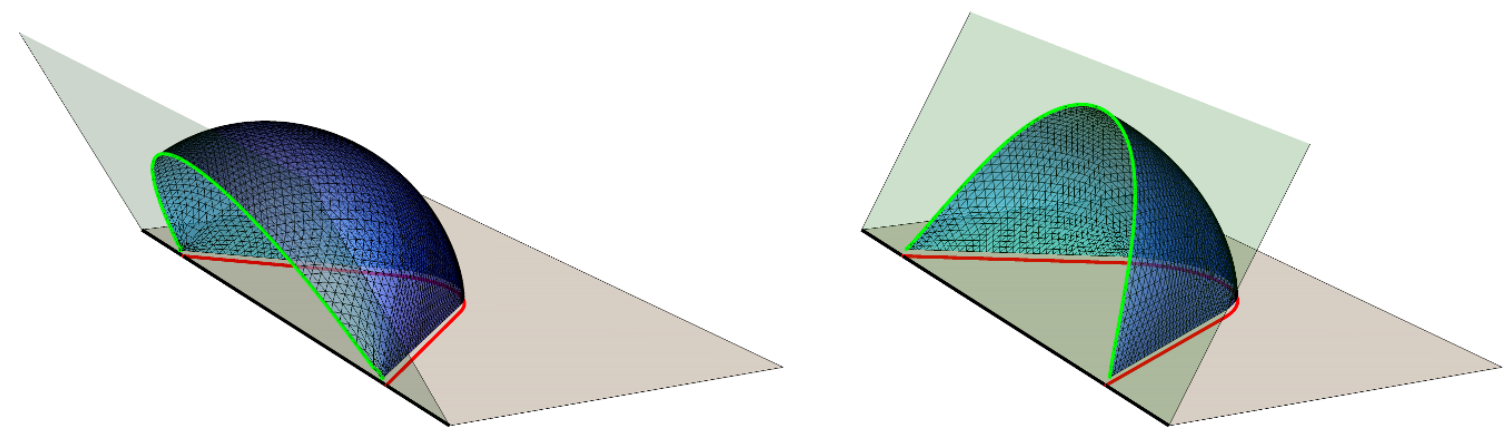

Figure 13. Minimal surfaces $\hat{\gamma}_{A}$ obtained with Surface Evolver corresponding to a region $A$ delimited by the red curve (entangling curve $\partial A \cap \partial B$ ) in the grey half plane at $z=0$, which has been obtained by smoothly joining two segments of equal length $L$ forming two equal corners with the boundary, whose opening angle is $\gamma$. The green half plane is $\mathcal{Q}$ defined by (4.3) and the green curve corresponds to $\hat{\gamma}_{A} \cap \mathcal{Q}$. In the left panel $\alpha=\pi / 3, L=1$ and $\gamma=0.8$, while in the right panel $\alpha=2 \pi / 3, L=1$ and $\gamma=1$. The numerical data of the corner function $F_{\alpha}(\gamma)$ corresponding to this kind of domains are labeled by empty triangles in figure 14 .

which is a special case of (1.9) and (1.10) with $P_{A, B}=L$ and $F_{\alpha, \text { tot }}=F_{\alpha}(\gamma)$. The leading linear divergence in (6.9) is the expected area law term and it comes from the part of $\hat{\gamma}_{\varepsilon}$ close the edge of $A$ at $\phi=\gamma$. The occurrence of the wedge leads to the important logarithmic divergence, whose coefficient provides the corner function $F_{\alpha}(\gamma)$ we are interested in.

The corner function $F_{\alpha}(\gamma)$ has been computed in the appendix F.3 and the result is

$$
F_{\alpha}=F\left(q_{0}\right)+\eta_{\alpha} \mathcal{G}\left(q_{*}\left(\alpha, q_{0}\right), q_{0}\right)
$$

where $F\left(q_{0}\right)$ has been introduced in $(3.3)$ and the function $\mathcal{G}\left(q, q_{0}\right)$ is

$$
\mathcal{G}\left(q, q_{0}\right) \equiv \sqrt{1+q_{0}^{2}}\left\{\mathbb{F}\left(\sigma\left(q, q_{0}\right) \mid-Q_{0}^{2}\right)-\mathbb{E}\left(\sigma\left(q, q_{0}\right) \mid-Q_{0}^{2}\right)+\sqrt{\frac{\left(q^{2}+1\right)\left(q^{2}-q_{0}^{2}\right)}{\left(q_{0}^{2}+1\right)\left(q^{2}+q_{0}^{2}+1\right)}}\right\}
$$

The expression for $q_{*}\left(\alpha, q_{0}\right)$ to use in (6.10) is (6.4).

The main result of this manuscript are (6.5) and (6.10), which provide the analytic expression of the corner function $F_{\alpha}(\gamma)$ in a parametric form in terms of $q_{0}>0$.

In figure 14 the solid curves corresponds to the corner function $F_{\alpha}(\gamma)$ for some values of $\alpha$. As for the argument of the corner function $F_{\alpha}(\gamma)$, we remind that $\gamma \in(0, \pi / 2]$. Nonetheless, whenever $\gamma \in(0, \pi)$ we mean $F_{\alpha}(\min [\gamma, \pi-\gamma])$.

In figure 15 we show the surface given by the corner function $F_{\alpha}(\gamma)$ in terms of the opening angle $\gamma$ and the slope $\alpha \in(0, \pi)$. In this figure we have highlighted the sections corresponding to the curves reported in figure 14 and also the curve $F_{\alpha}(\pi / 2)$ (yellow curve).

We have employed Surface Evolver to find an important numerical evidence of our analytic result. In this numerical analysis we have chosen domains $A$ whose entangling curves $\partial A \cap \partial B$ correspond to the red solid curves in the $z=0$ half plane shown in figure 12 and in figure 13. In particular, in figure 12 we have that $A$ is part of a disk which is not centered on the boundary and in figure 13 the region $A$ is made by two finite 


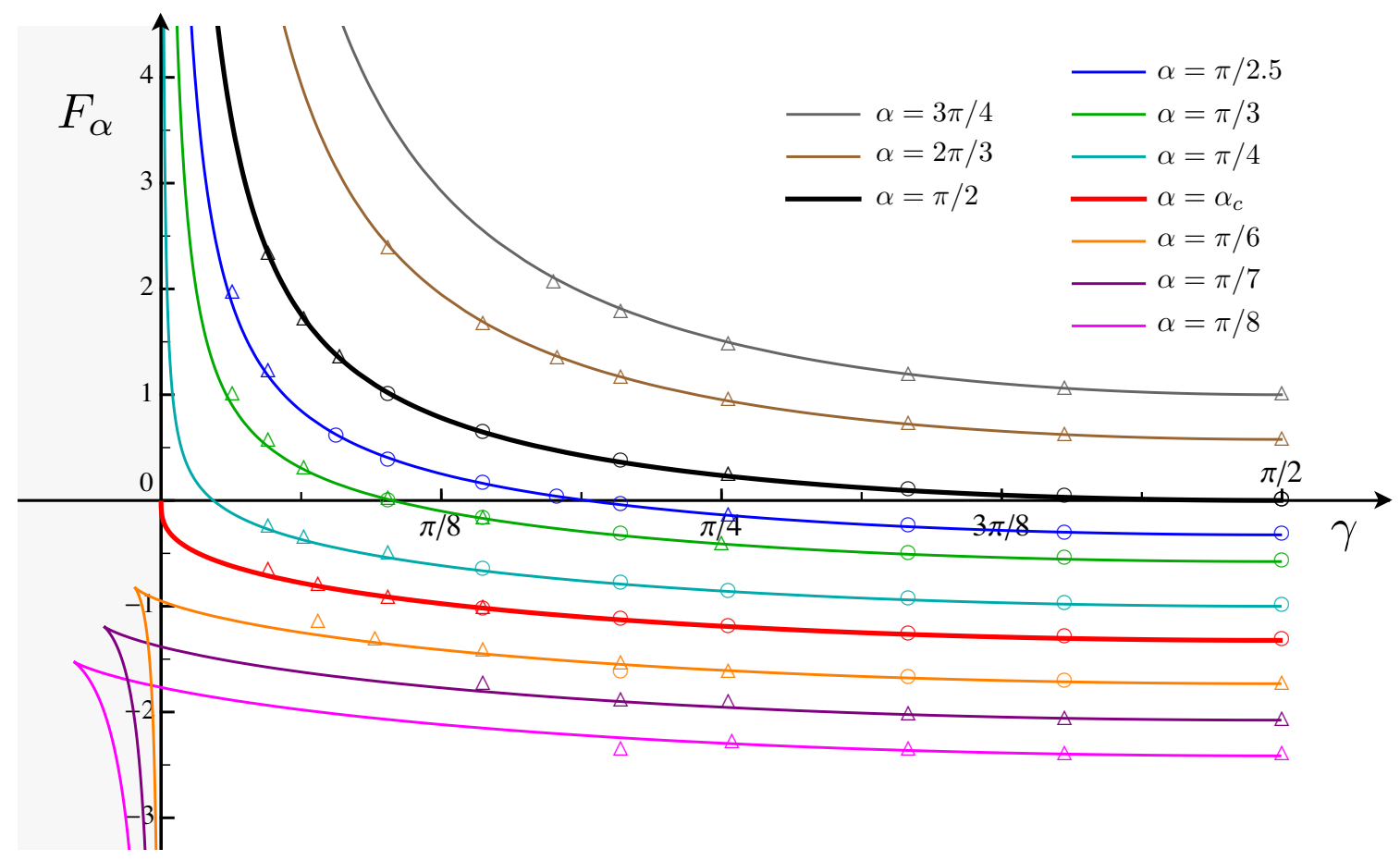

Figure 14. The corner function $F_{\alpha}(\gamma)$ for some values of the slope $\alpha$ of the half plane $\mathcal{Q}$. The solid curves are obtained from the analytic expressions (6.5) and (6.10), which provide the corner function parametrically in terms of $q_{0}>0$ (see also figure 15). The marked points have been found through our numerical analysis based on Surface Evolver. The empty circles label the data points obtained from the domain $A$ in figure 12, while empty triangles label the data points found by employing the domain $A$ in figure 13. The same color has been adopted for the analytic curves and the data points corresponding to the same $\alpha$.

wedges with an edge on the boundary and the same opening angle whose remaining edges are joined smoothly. These domains are simple finite regions with the smallest number of corners providing the corner function $F_{\alpha}(\gamma)$ we are interested in. In figure 12 and in figure 13 we show also the corresponding minimal surface $\hat{\gamma}_{A}$ constructed with Surface Evolver for a value $\alpha<\pi / 2$ (left panels) and for a value $\alpha>\pi / 2$ (right panels).

The marked points in figure 14 are the numerical values of the corner function $F_{\alpha}(\gamma)$ obtained through the numerical analysis based on the data obtained from Surface Evolver, as briefly explained in the appendix A. In particular, the empty circles and the empty triangles correspond to the domains $A$ shown in figure 12 and in figure 13 respectively. It turns out that the domain $A$ in figure 13 is more suitable to deal with small values of $\gamma$ in our numerical approach. Excellent agreement is obtained with the analytic result for the values of $\alpha$ and $\gamma$ considered in figure 14 .

From figure 14 and figure 15 we observe that for the holographic corner function given by (6.5) and (6.10) we have that $F_{\alpha}^{\prime}(\gamma) \leqslant 0$ and also $F_{\alpha}^{\prime \prime}(\gamma) \geqslant 0$ for any fixed value of the slope $\alpha \in(0, \pi)$. Furthermore, from figure 15 we also notice that $\partial_{\alpha} F_{\alpha}(\gamma)>0$ for any fixed value of $\gamma \in(0, \pi / 2]$. It would be interesting to understand whether these properties come from some more fundamental principles. 


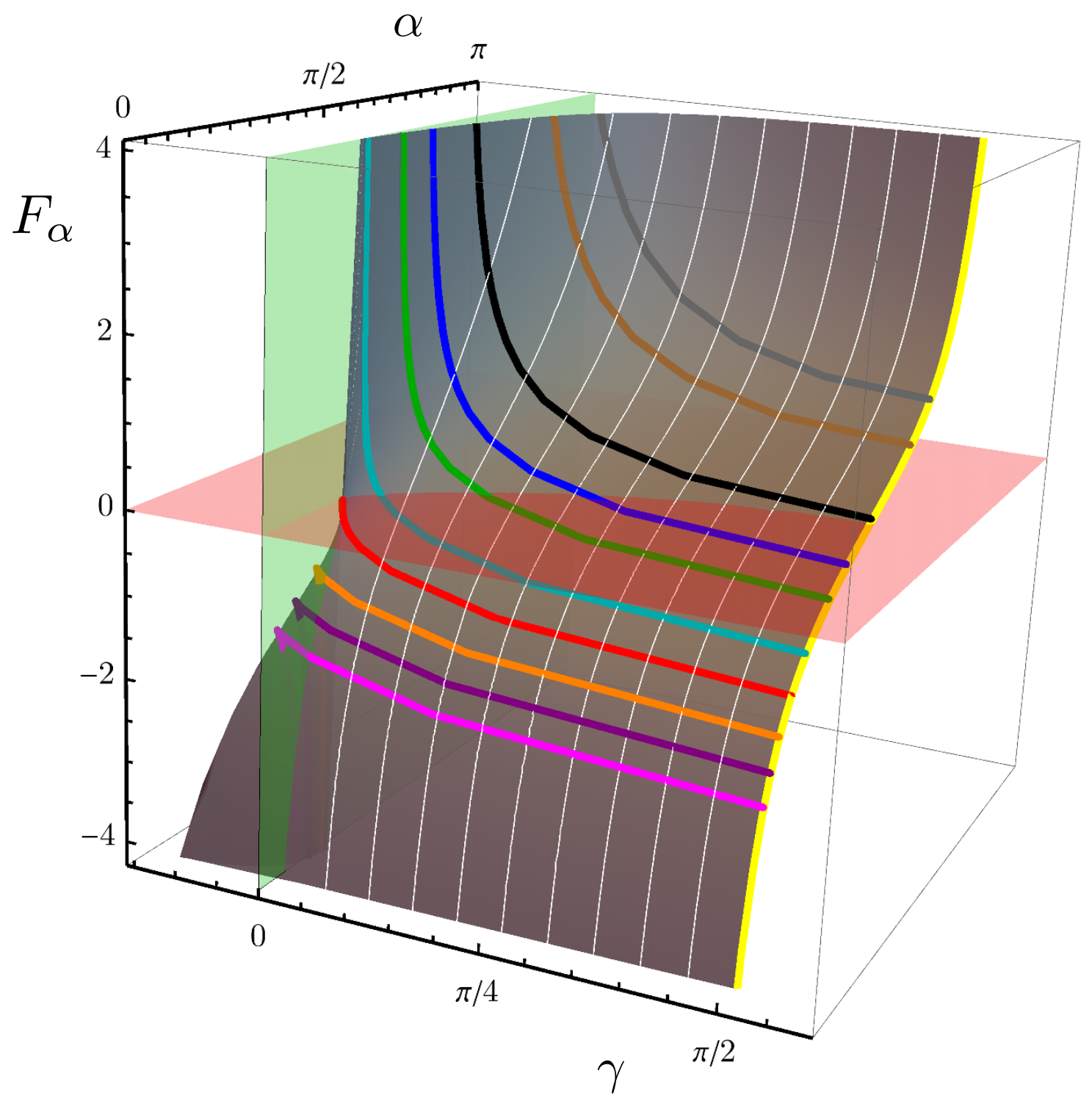

Figure 15. The corner function $F_{\alpha}(\gamma)$ given by (6.5) and (6.10) in terms of $\gamma \in(0, \pi / 2]$ and $\alpha \in(0, \pi)$ (grey surface). The solid curves corresponding to the $\alpha=$ const sections are the same ones shown in figure 14, with the same colour code. The section $\gamma=\pi / 2$ (yellow solid curve) is (5.2). In the left panel of figure 27 we depict the intersection between the grey surface and the red plane and in the right panel of figure 27 the intersection of the grey surface with the green plane is shown.

In figure 14 the curves corresponding to the critical value $\alpha=\alpha_{c}$ (red curve) given by (5.6) and to $\alpha=\pi / 2$ (black curve) have been highlighted by employing thicker lines because these values separate the range of $\alpha \in(0, \pi)$ into three intervals for $\alpha$ where the corner function $F_{\alpha}(\gamma)$ has different features. In particular, when $\alpha \geqslant \pi / 2$ we have that $F_{\alpha}(\gamma) \geqslant 0$, while when $\alpha \leqslant \alpha_{c}$ we have that $F_{\alpha}(\gamma) \leqslant 0$. In the intermediate range $\alpha \in$ $\left(\alpha_{c}, \pi / 2\right)$ the corner function does not have a definite sign in the whole range $\gamma \in(0, \pi / 2]$ and, being $F_{\alpha}^{\prime}(\gamma)<0$, it has a unique zero $\gamma=\gamma_{0}$. The value $\gamma_{0}$ in terms of $\alpha \in\left[\alpha_{c}, \pi / 2\right]$ found numerically is shown in the left panel of figure 27 . 
From figure 14 we observe that the corner function $F_{\alpha}(\gamma)$ displays two qualitative different behaviours as $\gamma \rightarrow 0^{+}$. Indeed, $F_{\alpha}(\gamma) \rightarrow+\infty$ when $\alpha>\alpha_{c}$, while it reaches a finite (non positive) value when $\alpha \leqslant \alpha_{c}$. In section 6.1 more quantitative results about the regimes $\gamma \rightarrow 0^{+}$and $\gamma \rightarrow \pi / 2$ of $F_{\alpha}(\gamma)$ are obtained.

It would be interesting to get a direct numerical confirmation of the occurrence of $\alpha_{c}$ through Surface Evolver or other methods. Unfortunately, we have not been able to push our numerical analysis to values of $\gamma$ small enough to appreciate the qualitatively different behaviour of the corner function for $\alpha \leqslant \alpha_{c}$ and $\alpha>\alpha_{c}$. Hopefully, this gap will be fixed in future studies.

\subsection{Limiting regimes of the corner function}

It is worth studying the corner function $F_{\alpha}(\gamma)$ in some particular regimes. In the following we report only the main results of our analysis, referring the reader to the appendices F.3 and F.4 for a detailed discussion of their derivations.

An important special value to consider is $\alpha=\pi / 2$. In this case it is straightforward to realise that the minimal surface $\hat{\gamma}_{A}$ is half of the auxiliary minimal surface $\hat{\gamma}_{A \text {,aux }}$ in $\mathbb{H}_{3}$, which is anchored to the auxiliary infinite wedge $A_{\text {aux }}$ with opening angle $2 \gamma$. Indeed, for every $\alpha$ we have that $\hat{\gamma}_{A \text {,aux }}$ in $\mathbb{H}_{3}$ is smooth and symmetric with respect to the half plane orthogonal to $z=0$ passing through the bisector of $A_{\text {aux }}$; therefore $\hat{\gamma}_{A \text {,aux }}$ intersects this half plane orthogonally. When $\alpha=\pi / 2$ the half plane characterising this reflection symmetry coincides with $\mathcal{Q}$. Thus, $\hat{\gamma}_{A \text {,aux }}$ is the union of $\hat{\gamma}_{A}$ and its reflected image with respect to $\mathcal{Q}$ obtained by sending $x \rightarrow-x$.

As for the corner function at $\alpha=\pi / 2$, from the analytic expression (6.5) and (6.10) we find respectively that

$$
\lim _{\alpha \rightarrow \pi / 2} \gamma=P_{0}\left(q_{0}\right) \quad \lim _{\alpha \rightarrow \pi / 2} F_{\alpha}=F\left(q_{0}\right)
$$

Further comments can be found in the closing remarks of the appendix F.3. Comparing (6.12) with (3.2) and (3.4) respectively, we obtain

$$
\widetilde{F}(2 \gamma)=2 F_{\pi / 2}(\gamma)
$$

Thus, the corner function found in [27] and discussed in section 3 is recovered as the special case $\alpha=\pi / 2$ of the corner function $F_{\alpha}(\gamma)$ given by (6.5) and (6.10).

We find it worth considering the corner function $F_{\alpha}(\gamma)$ in the limiting regimes of $\gamma \rightarrow 0$ and $\gamma \rightarrow \pi / 2$, which correspond to $q_{0} \rightarrow+\infty$ and $q_{0} \rightarrow 0^{+}$respectively, as discussed in the appendix F.4.

Taking the limit $q_{0} \rightarrow+\infty$ of (6.5) and (6.10), we obtain

$$
\gamma=\frac{\mathfrak{g}(\alpha)}{q_{0}}+O\left(1 / q_{0}^{3}\right) \quad F_{\alpha}=\mathfrak{g}(\alpha) q_{0}+O\left(1 / q_{0}\right) \quad q_{0} \rightarrow+\infty
$$

where $\mathfrak{g}(\alpha)$ is the function (5.4), given by the red curve in figure 10. In particular, $\gamma \rightarrow 0$ for large $q_{0}$. We remark that we have different behaviours of the corner function $F_{\alpha}(\gamma)$ as $\gamma \rightarrow 0^{+}$, depending on whether $\alpha \in\left(0, \alpha_{c}\right]$ or $\alpha \in\left(\alpha_{c}, \pi\right)$. Indeed, $\mathfrak{g}(\alpha)$ changes its sign 
at the critical value $\alpha=\alpha_{c}$ defined by (5.5), whose numerical value is (5.6). Since $\gamma$ and $q_{0}$ must be strictly positive, while $\mathfrak{g}(\alpha) \leqslant$ for $\alpha \in\left(0, \alpha_{c}\right]$, the expansion of $\gamma$ in (6.14) is meaningful in our setup only when $\alpha \in\left(\alpha_{c}, \pi\right)$. In this range, from the first expansion in (6.14) we find that $q_{0}=\mathfrak{g}(\alpha) / \gamma+O(\gamma)$ as $\gamma \rightarrow 0$. Then, plugging this result into the second expansion of (6.14), we obtain

$$
F_{\alpha}=\frac{\mathfrak{g}(\alpha)^{2}}{\gamma}+O(\gamma) \quad \gamma \rightarrow 0^{+} \quad \alpha \in\left(\alpha_{c}, \pi\right)
$$

When $\alpha=\alpha_{c}$ the second expansion in (6.15) tells us that

$$
F_{\alpha_{c}}(0)=0
$$

We can interpret this observation as a possible definition of $\alpha_{c}$ in terms of the corner function.

Notice that (6.16) suggests the following way to find $\alpha_{c}$ by employing finite domains. Consider for simplicity a domain $A$ with only two equal corners adjacent to the boundary, like e.g. in figure 13, and send their opening angles to zero simultaneously. In this limit the coefficient of the logarithmic divergence diverges when $\alpha>\alpha_{c}$ and tends to a finite value $F_{\alpha}(0)<0$ for $\alpha \leqslant \alpha_{c}$. The value $F_{\alpha}(0)$ corresponds to a finite value $\hat{q}_{0}$ of the parameter $q_{0}$ for a given $\alpha$. The function $F_{\alpha}(0)$ can be found numerically in terms of $\alpha \in\left(0, \alpha_{c}\right)$ and the result of this analysis is shown in the right panel of figure 27 in the appendix F. In particular, when $\alpha=\alpha_{c}$ we have (6.16).

From figure 14 we observe that in the range $\alpha \in\left[\alpha_{c}, \pi / 2\right)$ the function $F_{\alpha}(\gamma)$ vanishes at a positive value $\gamma_{0}$ of the opening angle. When $\alpha=\alpha_{c}$ we have $\gamma_{0}=0$, as written in (6.16). By solving numerically the equation $F_{\alpha}\left(\gamma_{0}\right)=0$ for $\alpha \in\left[\alpha_{c}, \pi / 2\right)$, we find the function $\gamma_{0}(\alpha)$ shown in the left panel of figure 27.

When $\alpha \in\left(0, \alpha_{c}\right)$ we have $\mathfrak{g}(\alpha)<0$; therefore the expansions in (6.14) imply that $\gamma \rightarrow 0^{-}$and $F_{\alpha} \rightarrow-\infty$ as $q_{0} \rightarrow+\infty$. Negative values of $\gamma$ are meaningless in our context. Nonetheless, from a mathematical perspective, we find it worth extending the domain of $\gamma$ to negative values. When $\gamma<0$ the parametric curve given by (6.5) and (6.10) does not provide a function of $\gamma$, but it is still a well defined curve. Indeed, from figure 14 it is straightforward to observe that, in the regime $\alpha<\alpha_{c}$, we have that $\gamma \rightarrow 0^{-}$when either $q_{0} \rightarrow \hat{q}_{0}$ or $q_{0} \rightarrow+\infty$. In the latter case we have that $F_{\alpha}=\mathfrak{g}(\alpha)^{2} / \gamma+O(\gamma)$.

In the appendix F.5 we explain the relation between the regime $\gamma \rightarrow 0^{+}$of the corner function $F_{\alpha}(\gamma)$ and the holographic entanglement entropy (5.9) of the infinite strip adjacent to the boundary. This is due to the existence of a conformal map which relates the infinite wedge with $\gamma \rightarrow 0^{+}$to an infinite strip. The discussion reported in the appendix F.5 is a modification of the analogue one in $\mathrm{AdS}_{4} / \mathrm{CFT}_{3}$ [41, 59, 93, 94], obtained by taking into account the presence of the boundary in a straightforward way.

As for the regime $q_{0} \rightarrow 0^{+}$, in the appendix F.4.2 we have computed the expansions of the opening angle $\gamma$ and of the corner function $F_{\alpha}$, which are given by (6.5) and (6.10) respectively, finding (F.47) and (F.51) respectively. From these results we can conclude that $\gamma \rightarrow \pi / 2$ and also that

$$
F_{\alpha}(\gamma)=-\cot \alpha+\frac{(\pi / 2-\gamma)^{2}}{2(\pi-\alpha)}+O\left((\pi / 2-\gamma)^{4}\right)
$$


which agrees with the general expansion (2.2) for this kind of corner function. In particular, we have that with $F_{\alpha}(\pi / 2)=-\cot \alpha$ and $F_{\alpha}^{\prime \prime}(\pi / 2)=1 /(\pi-\alpha)$. The expression for $F_{\alpha}(\pi / 2)$ confirms the expected result (5.2) obtained in section 5.1 by considering the half disk centered on the boundary. Let us remark that the method discussed in the appendix F.4 allows to computed also higher orders in (6.17). For instance, in (F.52) also the $O((\pi / 2-$ $\gamma)^{4}$ ) term has been reported.

\subsection{Relations with the stress tensor}

We find it worth exploring possible universal relations among the corner functions and other quantities of the underlying $\mathrm{BCFT}_{3}$ model.

In $\mathrm{CFT}_{3}$, an important example of universal relation involves the corner function $\tilde{f}(\theta)$ and the two point function of the stress tensor $T_{\mu \nu}$, which is given by $\left\langle T_{\mu \nu}(x) T_{\rho \sigma}(0)\right\rangle=$ $\left(C_{T} /|x|^{6}\right) \mathcal{I}_{\mu \nu, \rho \sigma}(x)$, being $\mathcal{I}_{\mu \nu, \rho \sigma}(x)$ a dimensionless tensor structure fixed by symmetry. In particular, by considering the coefficient $\tilde{\sigma}=\tilde{f}^{\prime \prime}(\pi) / 2$ of the leading term in the expansion $\tilde{f}(\theta)=\tilde{\sigma}(\pi-\theta)^{2}+\ldots$ as $\theta \rightarrow \pi^{-}$, it has been found that $[40,41]$

$$
\frac{\tilde{\sigma}}{C_{T}}=\frac{\pi^{2}}{24}
$$

In $\mathrm{AdS}_{4} / \mathrm{CFT}_{3}$ the holographic corner function is $\tilde{f}(\theta)=\frac{L_{\mathrm{AdS}}^{2}}{4 G_{\mathrm{N}}} \widetilde{F}(\theta)$, as discussed in section 1 . Denoting by $\tilde{\sigma}_{\mathrm{E}}$ the coefficient $\tilde{\sigma}$ for this holographic corner function in a bulk theory described by Einstein gravity, we have that $\tilde{\sigma}_{\mathrm{E}}=\frac{L_{\mathrm{AdS}}^{2}}{8 G_{\mathrm{N}}} \widetilde{F}^{\prime \prime}(\pi)$. Considering the corner function $F_{\alpha}(\gamma)$ in $\mathrm{AdS}_{4} / \mathrm{BCFT}_{3}$ given by (6.5) and (6.10), in section 6.1 the relation (6.12) has been observed when $\alpha=\pi / 2$. Taking the limit $\gamma \rightarrow \pi / 2$ of (6.12) by employing (2.2) and $F_{\pi / 2}\left(\frac{\pi}{2}\right)=0$, one finds that $2 \widetilde{F}^{\prime \prime}(\pi)=F_{\pi / 2}^{\prime \prime}\left(\frac{\pi}{2}\right)$. The latter relation and $F_{\alpha}^{\prime \prime}\left(\frac{\pi}{2}\right)=$ $1 /(\pi-\alpha)($ see $(6.17))$ evaluated for $\alpha=\pi / 2$ provide $\sigma_{\mathrm{E}}=\frac{L_{\mathrm{AdS}}^{2}}{16 G_{\mathrm{N}}} F_{\pi / 2}^{\prime \prime}\left(\frac{\pi}{2}\right)=\frac{L_{\mathrm{AdS}}^{2}}{16 \pi G_{\mathrm{N}}} 2$. Then, by employing the holographic result $C_{T}=3 L_{\text {AdS }}^{2} /\left(\pi^{3} G_{\mathrm{N}}\right)=\frac{L_{\text {AdS }}^{2}}{16 \pi G_{\mathrm{N}}}\left(48 / \pi^{2}\right)$ found in $[95,96]$, one obtains $\tilde{\sigma}_{\mathrm{E}} / C_{T}=\pi^{2} / 24$, which corresponds to (6.18) in the holographic setup determined by the Einstein gravity in the bulk. Thus, consistency has been found between (6.12) and the ratio (6.18).

We find it interesting to explore the possibility that universal relations exist also for $\mathrm{BCFT}_{3}$.

Considering the two dimensional manifold $\partial \mathcal{B}$ given by the boundary of a $\mathrm{BCFT}_{3}$ defined in the spacetime $\mathcal{B}$, let us denote by $b_{i j}$ and $k_{i j}$ the metric induced on $\partial \mathcal{B}$ and its extrinsic curvature respectively. By introducing the trace $k$ of the extrinsic curvature, the combination $\kappa_{i j} \equiv k_{i j}-(k / 2) b_{i j}$ gives the traceless part of $k_{i j}$.

In a $\mathrm{BCFT}_{3}$, the presence of the boundary leads to a non trivial Weyl anomaly localised on the boundary. It is given by $[49,50]$

$$
\left\langle T_{i}^{i}\right\rangle=\frac{1}{4 \pi}\left(-\mathfrak{a} \mathcal{R}+\mathfrak{q} \operatorname{Tr} \kappa^{2}\right) \delta(\partial \mathcal{B})
$$

where $\delta(\partial \mathcal{B})$ is the Dirac delta whose support is $\partial \mathcal{B}$. In (6.19) we have that $\mathcal{R}$ is the Ricci scalar corresponding to the metric $b_{i j}$ induced on $\partial \mathcal{B}$ and $\operatorname{Tr} \kappa^{2} \equiv \kappa^{i j} \kappa_{i j}$. The constants $\mathfrak{a}$ 
and $\mathfrak{q}$ are the boundary central charges, which depends on the underlying model and also on the conformally invariant boundary conditions characterising the $\mathrm{BCFT}_{3}$. They have been computed for some free models in $[49,56,65]$. The quantity $\left\langle T_{i}^{i}\right\rangle$ has been studied also in $\mathrm{BCFT}_{4}[51,52]$.

We also need to consider the behaviour of the one point function of the stress tensor in the $\mathrm{BCFT}_{3}$ near $\partial \mathcal{B}$. In terms of the proper distance $X$ from $\partial \mathcal{B}$, it is given by [97]

$$
\left\langle T_{i j}\right\rangle=\frac{A_{T}}{X^{2}} \kappa_{i j}+\ldots \quad X \rightarrow 0^{+}
$$

where $\kappa_{i j}$ is the traceless part of the extrinsic curvature of the boundary and the coefficient $A_{T}$ depends on the conformally invariant boundary conditions of the underlying $\mathrm{BCFT}_{3}$.

Notice that in the $\mathrm{BCFT}_{4}$ given by a scalar field, the coefficient $A_{T}$ has been computed in [97] and the same negative value has been obtained for both Dirichlet and Neumann boundary conditions. We have not found in the literature an explicit computation of $A_{T}$ for a $\mathrm{BCFT}_{3}$.

Let us focus on the holographic corner function $f_{\alpha}(\gamma)=\frac{L_{\mathrm{AdS}}^{2}}{4 G_{\mathrm{N}}} F_{\alpha}(\gamma)$, where $F_{\alpha}(\gamma)$ is given by (6.5) and (6.10).

Let us recall that in the AdS/BCFT construction discussed in [63], the Neumann boundary conditions given by $K_{a b}=(K-T) h_{a b}$ have been imposed to define the hypersurface $\widetilde{\mathcal{Q}}$ in the bulk delimiting the gravitational spacetime. Instead, in [72-74] it has been proposed to employ the less restrictive boundary condition $K=\frac{d+1}{d} T$ to find $\widetilde{\mathcal{Q}}$. When the boundary of the $\mathrm{BCFT}_{3}$ is flat, both these prescriptions provides the half plane $\widetilde{\mathcal{Q}}=\mathcal{Q}$ given by (4.3).

In the AdS/BCFT setup of [63], by considering a $\mathrm{BCFT}_{3}$ defined on the three dimensional sphere (in the Euclidean signature), whose boundary is a two dimensional sphere for which $\kappa_{i j}$ vanishes, it has been found that $[64]^{4}$

$$
\mathfrak{a}=\frac{L_{\operatorname{AdS}}^{2}}{4 G_{\mathrm{N}}}(-\cot \alpha)
$$

which means that $\mathfrak{a}=f_{\alpha}(\pi / 2)$. By using instead the boundary conditions $K=\frac{d+1}{d} T$, the relations $\mathfrak{q}=\mathfrak{a}=f_{\alpha}(\pi / 2)$ have been obtained [72-74]. Notice that the relation $\mathfrak{q}=\mathfrak{a}$ is not true for a free scalar $[49,56,65]$.

We remark that, since the holographic corner function given by (6.5) and (6.10) has been found for a flat boundary, it should be same for both the above $\mathrm{AdS}_{4} / \mathrm{BCFT}_{3}$ constructions, once the prescription (4.5) for the holographic entanglement entropy is accepted.

Given the holographic result (6.21), one could wonder whether $f_{\alpha}(\pi / 2)=\mathfrak{a}$ holds for any $\mathrm{BCFT}_{3}$. In [56] it has been shown that this relation fails for the scalar field because of the occurrence of a non minimal coupling to the curvature. Checking the validity of $f_{\alpha}(\pi / 2)=\mathfrak{a}$ for other models is an interesting issue for future studies.

In the remaining part of this section we explore a relation involving the coefficient $f_{\alpha}^{\prime \prime}(\pi / 2)$ of the expansion (2.2) of the holographic corner function as $\gamma \rightarrow \pi / 2$ and the

\footnotetext{
${ }^{4}$ Comparing with the notation of [64], we find that $\left.\left(-c_{b d y} / 6\right)\right|_{\text {there }}=\mathfrak{a}$.
} 
coefficient $A_{T}$ introduced in (6.20) by considering the one point function of the stress tensor close to the boundary $\partial \mathcal{B}$.

In $\mathrm{AdS}_{4} / \mathrm{BCFT}_{3}$ we found that $F_{\alpha}^{\prime \prime}(\pi / 2)=1 /(\pi-\alpha)$ for $\alpha \in(0, \pi)$ (see $(6.17)$ ); therefore we have

$$
f_{\alpha}^{\prime \prime}(\pi / 2)=\frac{L_{\mathrm{AdS}}^{2}}{16 \pi G_{\mathrm{N}}} \frac{4 \pi}{\pi-\alpha}
$$

By employing the AdS/BCFT construction of [63] and the standard approach to the holographic stress tensor discussed in [84-86], in the appendix $\mathrm{G}$ we have revisited the analysis of $[98]^{5}$ finding the expression of $A_{T}$ in $\mathrm{AdS}_{d+2} / \mathrm{BCFT}_{d+1}$ with the boundary conditions of [63] (see (G.27)). In the special case of $d=2$, for $\alpha \in(0, \pi)$ we obtain

$$
A_{T}=-\frac{L_{\mathrm{AdS}}^{2}}{16 \pi G_{\mathrm{N}}} \frac{2}{\pi-\alpha}
$$

From (6.22) and (6.23), we find it interesting to observe that in the $\mathrm{AdS}_{4} / \mathrm{BCFT}_{3}$ setup of [63] the ratio $f_{\alpha}^{\prime \prime}(\pi / 2) / A_{T}$ is independent of the slope $\alpha$, which could be related to the conformally invariant boundary conditions allowed for the dual $\mathrm{BCFT}_{3}$. In particular this ratio reads

$$
\frac{f_{\alpha}^{\prime \prime}(\pi / 2)}{A_{T}}=-2 \pi
$$

We find it very interesting to compute the ratio (6.24) also for explicit models of three dimensional conformal field theories with boundary and for different boundary conditions. Free quantum field theories are the simplest models to address in this direction.

\section{Infinite wedge with only the tip on the boundary}

In this section we consider the domain given by an infinite wedge having its tip on the boundary whose edges do not belong to it. As discussed in section 1, in a generic $\mathrm{BCFT}_{3}$ the entanglement entropy of this region contains a logarithmic divergence whose coefficient provides a corner function $\mathrm{F}_{\alpha}(\vec{\omega})$ which cannot be determined from the corner function $f_{\alpha}(\gamma)$ corresponding to the infinite wedge adjacent to the boundary. In the following we explain that for the holographic entanglement entropy in $\mathrm{AdS}_{4} / \mathrm{BCFT}_{3}$ this analysis significantly simplifies and the corner function $\mathcal{F}_{\alpha}(\omega, \gamma)$ corresponding to this kind of wedge (see $(1.10)$ ) can be written in a form which involves the corner function $F_{\alpha}(\gamma)$ presented in section 6 and the corner function $\widetilde{F}(\theta)$ reviewed in section 3 .

Let us consider the infinite wedge $A$ with opening angle $\omega<\pi$ which has only the tip on the boundary $x=0$. Domains containing this kind of corner occur in figure 2, where they are labeled by $C$ and $C_{j}$. Setting the origin of the Cartesian coordinates in the tip of the wedge $A$, we have that the boundary $x=0$ is splitted into two half lines corresponding to $y<0$ and $y>0$. Denoting by $\gamma<\pi$ and $\tilde{\gamma}<\pi$ the opening angles of the corners in $B$, the supplementarity condition $\omega+\gamma+\tilde{\gamma}=\pi$ holds. We can assume that $\gamma \leqslant \tilde{\gamma}$ without loss of generality. Combining this inequality with the supplementarity condition,

\footnotetext{
${ }^{5}$ In the appendix G the differences between our results and the ones obtained in [98] are discussed.
} 

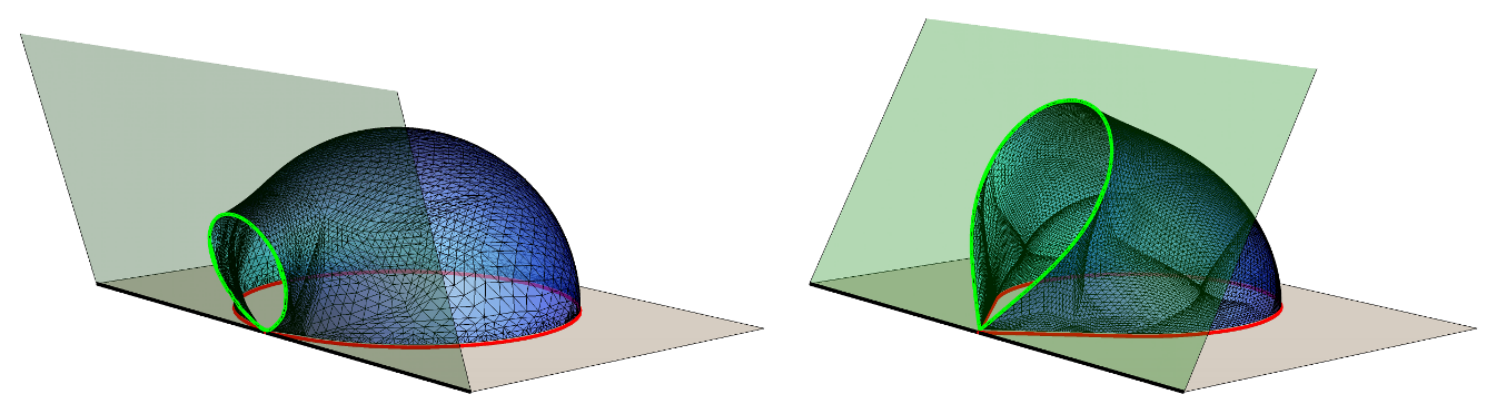

Figure 16. Minimal surfaces $\hat{\gamma}_{A}$ obtained with Surface Evolver and anchored to a single drop $A$ (whose boundary is the red solid curve in the $z=0$ grey half plane) which has only the tip on the boundary. Here $A$ has been chosen in a symmetric way (i.e. $\tilde{\gamma}=\gamma$ ). In the left panel $\alpha=\pi / 2.5$, $\omega=2.6$ and $L=0.5$, while in the right panel $\alpha=2 \pi / 3, \omega=\pi / 2$ and $L=1.5$. In both panels $\varepsilon=0.03$. This kind of minimal surfaces have been constructed to find the data corresponding to $\omega>\omega_{c}$ in figure 17, which have been labeled by empty black circles.

it is straightforward to observe that $\gamma \leqslant(\pi-\omega) / 2$. Instead, since $\tilde{\gamma}$ is not restricted, we have that $\tilde{\gamma} \in(0, \pi)$. In the following we denote by $L \gg \varepsilon$ the length of the edges of $A$, as done in section 6 for the wedge adjacent to the boundary.

Since the edges of $A$ do not belong to the boundary $x=0$, the minimal surface $\hat{\gamma}_{A}$ is anchored to both of them. Moreover, the expansion of the area of $\hat{\gamma}_{\varepsilon}$ is (1.9) with $P_{A, B}=2 L$ and the coefficient of the logarithmic divergence (1.10) given by $F_{\alpha, \text { tot }}=\mathcal{F}_{\alpha}(\omega, \gamma)$.

It is not difficult to realise that there are two candidates for $\hat{\gamma}_{A}$ which are local solutions of the minimal area condition in presence of $\mathcal{Q}$. The first one is a surface $\hat{\gamma}_{A}^{\text {dis }}$ which connects the two edges of $A$ through the bulk and is disconnected from the half plane $\mathcal{Q}$. Since $\hat{\gamma}_{A}^{\text {dis }} \cap \mathcal{Q}=\emptyset$, we have that $\hat{\gamma}_{A}^{\text {dis }}$ is the minimal area surface found in [27], which has been discussed in section 3 . The second solution is a surface $\hat{\gamma}_{A}^{\text {con }}$ which connects the two edges of $A$ to $\mathcal{Q}$ through the bulk. It is given by the union of two disjoint surfaces where each of them is like the one found in section 6 ; therefore $\hat{\gamma}_{A}^{\text {con }} \cap \mathcal{Q}$ is made by two half lines departing from the tip of the wedge.

The area $\mathcal{A}\left[\hat{\gamma}_{\varepsilon}\right]$, which provides the holographic entanglement entropy for this infinite wedge $A$, is the minimum between the area of $\hat{\gamma}_{A}^{\text {dis }} \cap\{z \geqslant \varepsilon\}$ and the area of $\hat{\gamma}_{A}^{\text {con }} \cap\{z \geqslant \varepsilon\}$. Being $P_{A, B}=2 L$ for both $\hat{\gamma}_{A}^{\text {dis }}$ and $\hat{\gamma}_{A}^{\text {con }}$, the minimal area surface $\hat{\gamma}_{A}$ must be found by comparing the coefficients of the subleading logarithmic divergence. This comparison leads to the following corner function

$$
\mathcal{F}_{\alpha}(\omega, \gamma)=\max \left\{\widetilde{F}(\omega), F_{\alpha}(\gamma)+F_{\alpha}(\tilde{\gamma})\right\} \quad \tilde{\gamma}=\pi-(\omega+\gamma)
$$

where the first function within the parenthesis corresponds to $\hat{\gamma}_{A}^{\text {dis }}$ and the second one to $\hat{\gamma}_{A}^{\text {con }}$. The corner function $\widetilde{F}(\omega)$ is the one found in [27] and reviewed in section 3 , while $F_{\alpha}(\gamma)$ is the corner function discussed in section 6 . Let us remind that, since $\tilde{\gamma} \in(0, \pi)$ in $(7.1)$ we mean $F_{\alpha}(\tilde{\gamma})=F_{\alpha}(\min [\tilde{\gamma}, \pi-\tilde{\gamma}])$, as stated in section 2 .

It could be useful to compare (7.1) with (3.7). Indeed, by extending the half plane $x \geqslant 0$ to the whole $\mathbb{R}^{2}$ and including the reflected image of $A$ obtained by sending $x \rightarrow-x$, 

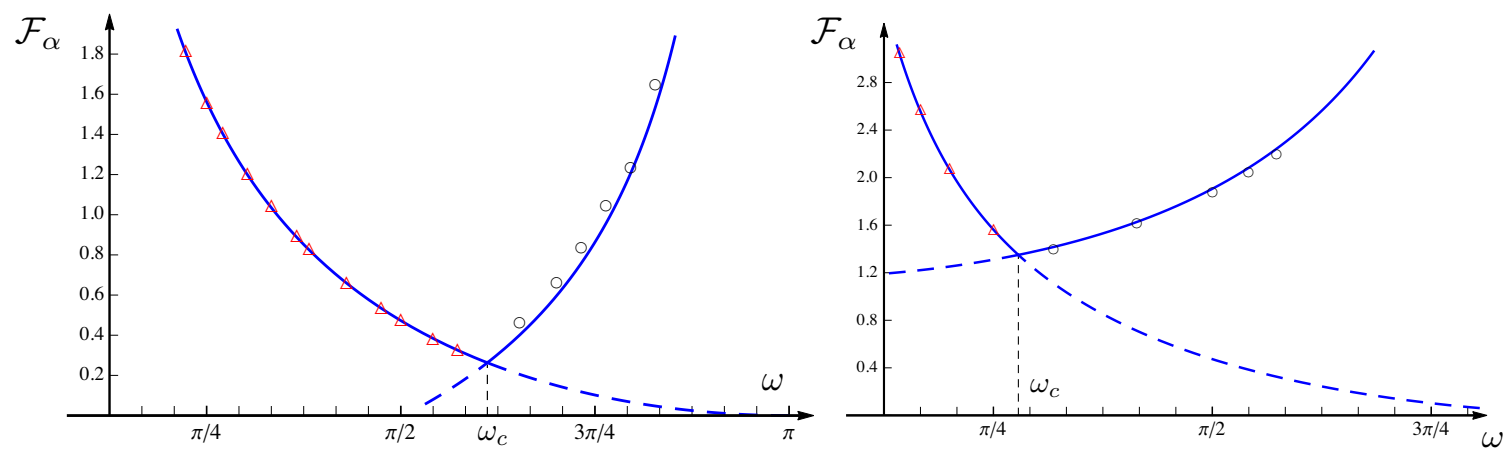

Figure 17. The corner function (7.2) for symmetric configurations of the infinite wedge (i.e. $\tilde{\gamma}=\gamma$ ). The slope $\alpha$ of $\mathcal{Q}$ is different in the two panels: $\alpha=\pi / 2.3$ (left) and $\alpha=2 \pi / 3$ (right). The solid blue line is obtained from the analytic expression (7.2). The data points have been found by constructing minimal surfaces with Surface Evolver anchored to single drop domains whose opening angle of the corner is $\omega$. The minimal surfaces corresponding to the empty black circles are connected to $\mathcal{Q}$ (see e.g. figure 16), while the ones corresponding to the empty red triangles are disconnected from $\mathcal{Q}$. The critical value $\omega_{c}$ is defined by (7.3). Notice that $\omega_{c}>\pi / 2$ when $\alpha<\pi / 2$ and $\omega_{c}<\pi / 2$ when $\alpha>\pi / 2$.

one obtains the symmetric configuration of corners underlying (3.7). Nonetheless, let us stress that (7.1) with (3.7) are not equivalent because in (7.1) the boundary conditions (which correspond to $\alpha$ in this holographic setup) play a central role.

The corner function (7.1) occurs in the constraints from the strong subadditivity found in section 2. In the appendix $\mathrm{H}$ we show that the holographic corner functions $F_{\alpha}(\gamma)$ and $\mathcal{F}_{\alpha}(\omega, \gamma)$ fulfils these constraints.

For the sake of simplicity, let us consider first the subclass of infinite wedges which are symmetric with respect to the half line departing from the tip and orthogonal to the boundary. For these wedges $\tilde{\gamma}=\gamma$; therefore the supplementarity condition implies that $\gamma=(\pi-\omega) / 2$. Thus, these configurations are fully determined by $\omega$ (equivalently, one can adopt $\gamma$ as independent variable). By substituting $\omega=\pi-2 \gamma$ into (7.1), we find that for these symmetric wedges the corner function simplifies to

$$
\mathcal{F}_{\alpha}(\omega, \gamma)=\max \left\{\widetilde{F}(\omega), 2 F_{\alpha}(\gamma)\right\} \quad \gamma=\frac{\pi-\omega}{2}
$$

The maximisation procedure occurring in (7.1) and (7.2) chooses the first function for some configurations and the second function for other ones. In particular, there exist critical configurations such that the two functions in the r.h.s.'s of (7.1) and (7.2) provide the same result, namely both $\hat{\gamma}_{A}^{\text {dis }}$ and $\hat{\gamma}_{A}^{\text {con }}$ have the same coefficient of the logarithmic divergence.

In figure 16 we show two examples of minimal area surfaces obtained with Surface Evolver which correspond to single drop domains $A$ (see section 3.1) whose corners have the tip on the boundary and belong to this class of symmetric wedges having $\tilde{\gamma}=\gamma$. In a neighbourhood of the tips of these two domains the minimal area surface $\hat{\gamma}_{A}$ is given by $\hat{\gamma}_{A}^{\text {con }}$.

In figure 17 the corner function (7.2) is plotted as function of $\omega$ for two particular values of $\alpha$. The critical value $\omega_{c}$, where the two functions in the r.h.s. of (7.2) are equal, is highlighted by the vertical dashed segments and it depends on the slope $\alpha$. For $\omega<\omega_{c}$ the 


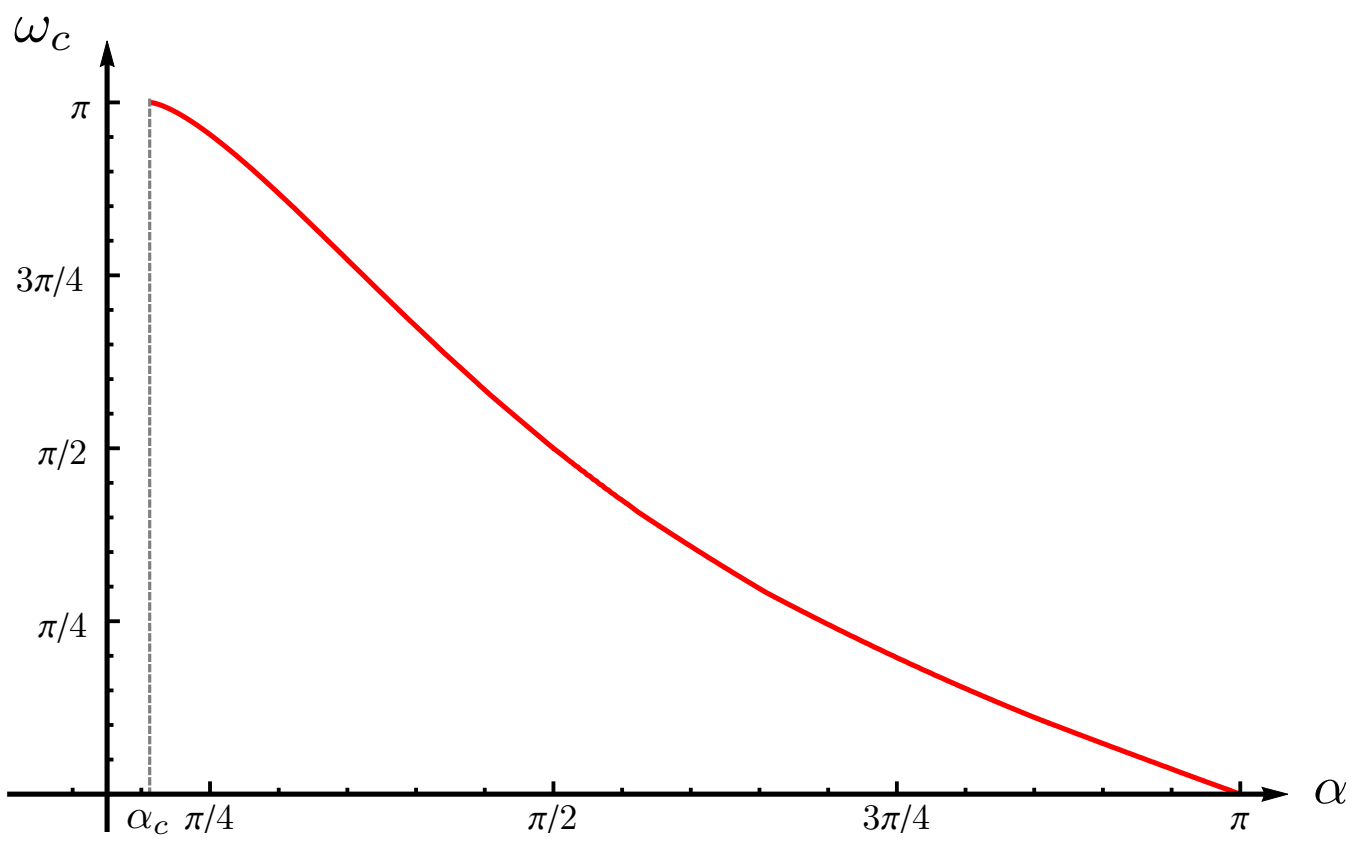

Figure 18. Infinite wedge with only the tip on the boundary and $\tilde{\gamma}=\gamma$ : the critical opening angle $\omega_{c}$ as function of $\alpha \geqslant \alpha_{c}$. The curve has been found by solving (7.3) numerically.

minimal surface $\hat{\gamma}_{A}$ is disconnected from $\mathcal{Q}$ and it is like the one shown in figure 3 , while for $\omega>\omega_{c}$ it is connected to $\mathcal{Q}$ and it looks like the minimal surfaces depicted in figure 16 . The minimal surfaces in figure 16 are prototypical examples of the surfaces employed to find the numerical data corresponding to the empty circles in figure 17.

By applying the remark made above about (7.1) to this simpler situation, it could be instructive to compare (7.2) with (3.6), which has been found for the analogous situation in $\mathrm{AdS}_{4} / \mathrm{CFT}_{3}$, as it can be observed by using the image method. Nonetheless, we remark again that in (7.2) the parameter $\alpha$ enters in a crucial way. By performing the same analysis done for figure 17 setting $\alpha=\pi / 2$, we have checked numerically the data shown in figure 6 are consistent with the relation (6.13).

In the remaining part of this section we describe the critical configurations corresponding to (7.1) and to (7.2).

Let us consider first the class of symmetric wedges where $\tilde{\gamma}=\gamma$. From (7.2), we have that the critical configuration is characterised by the opening angle $\omega_{c}=\omega_{c}(\alpha)$ which solves the following equation

$$
\widetilde{F}\left(\omega_{c}\right)=2 F_{\alpha}\left(\left(\pi-\omega_{c}\right) / 2\right)
$$

As consistency check we can set $\alpha=\pi / 2$. In this case, by employing (6.13) in the r.h.s. of (7.3), the equation (7.3) becomes $\widetilde{F}\left(\omega_{c}\right)=\widetilde{F}\left(\pi-\omega_{c}\right)$, whose solution is $\omega_{c}=\pi / 2$, as expected from the general fact the results in $\mathrm{AdS}_{4} / \mathrm{CFT}_{3}$ (see figure 6 for this quantity) are recovered in our $\mathrm{AdS}_{4} / \mathrm{BCFT}_{3}$ setup for $\alpha=\pi / 2$.

We find it worth focussing also on the special value $\alpha=\alpha_{c}$. By employing the characteristic property of $\alpha_{c}$ given by (6.16) and the fact that $\widetilde{F}(\pi)=0$ into (7.3), we find

$$
\lim _{\alpha \rightarrow \alpha_{c}} \omega_{c}(\alpha)=\pi
$$



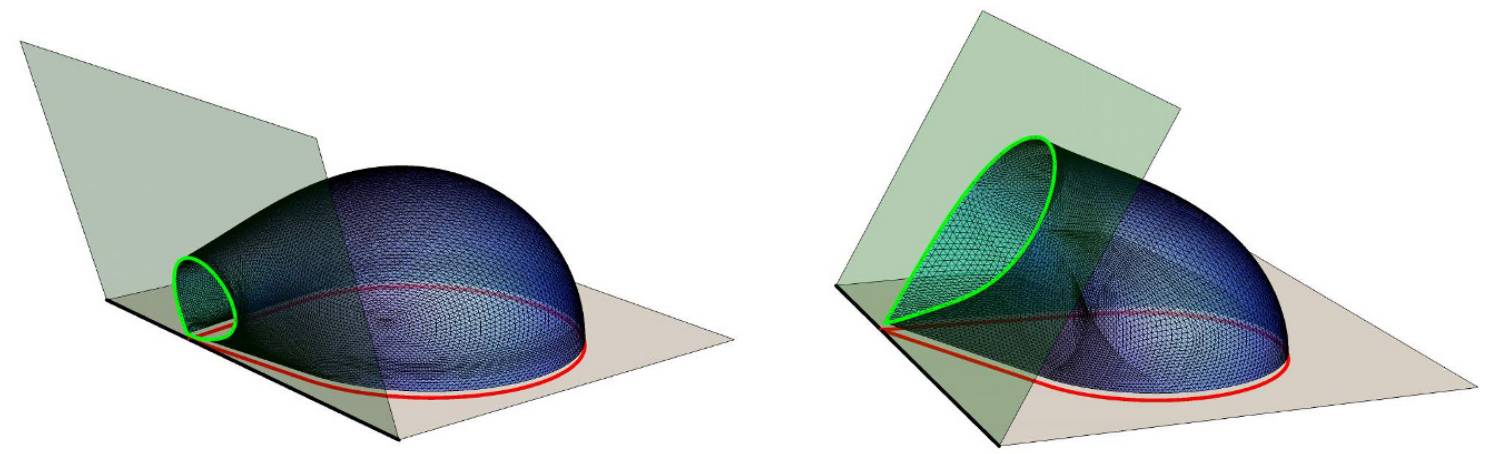

Figure 19. Minimal surfaces $\hat{\gamma}_{A}$ obtained with Surface Evolver and corresponding to a single drop $A$ such that the entangling curve $\partial A$ (solid red curve in the $z=0$ grey half plane) intersects the boundary at the tip of its corner. For these configurations of $A$, the corresponding minimal surface is the surface which intersects $\mathcal{Q}$ (green half plane) orthogonally along the green curve. In the left panel $\alpha=\pi / 2.5, \omega=\pi / 2, \gamma=\pi / 2-\pi / 5$ and $L=0.75$, while in the right panel $\alpha=2 \pi / 3, \omega=\pi / 3$, $\gamma=\pi / 2-\pi / 5$ and $L=1$. In both panels $\varepsilon=0.03$.

Since $\omega<\pi$, the limit (7.4) tells us that, within the class of symmetric wedges with $\tilde{\gamma}=\gamma$, the minimal area surface $\hat{\gamma}_{A}$ is always $\hat{\gamma}_{A}^{\text {dis }}$ when $\alpha \leqslant \alpha_{c}$. This observation can be inferred also from $(7.2)$ because $F_{\alpha}(\gamma) \leqslant 0$ for $\alpha \leqslant \alpha_{c}$, while $\widetilde{F}(\omega) \geqslant 0$. Thus, when $\alpha \leqslant \alpha_{c}$, the transition from $\hat{\gamma}_{A}=\hat{\gamma}_{A}^{\text {dis }}$ to $\hat{\gamma}_{A}=\hat{\gamma}_{A}^{\text {con }}$ as $\omega$ increases does not occur. The absence of this transition is a characteristic feature of the regime $\alpha \leqslant \alpha_{c}$ that can be detect with finite domains. We have not been able to get reliable numerical data from Surface Evolver for values of alpha close enough to $\alpha_{c}$; therefore we have not observed (7.4) numerically. Hopefully, future analysis will address this numerical issue.

In figure 18 we show the curve $\omega_{c}(\alpha)$ of the critical opening angle for the symmetric wedges, which has been obtained by solving (7.3) numerically. Notice that the curve lies above the straight line tangent to it and passing through the point $\alpha=\pi / 2$.

In the general case $\tilde{\gamma} \geqslant \gamma$ and the configuration of the infinite wedge is characterised by the independent angles $\gamma$ and $\omega$. In figure 19 we show the minimal area surfaces constructed with Surface Evolver which are anchored to two different configurations of a single drop domains $A$ having the tip on the boundary and with $\tilde{\gamma}>\gamma$. For the configurations in figure 19, the minimal area surface $\hat{\gamma}_{A}$ in the neighbourhood of the tip is given by $\hat{\gamma}_{A}^{\text {con }}$.

As discussed above, critical configurations exist such that the two functions involved in the maximisation procedure of (7.1) have the same value. For a given slope $\alpha$, we can equivalently characterise these configurations either by the critical value $\omega_{c}=\omega_{c}(\gamma, \alpha)$ in terms of $\gamma$ or by the critical value $\gamma_{c}=\gamma_{c}(\omega, \alpha)$ in terms of $\omega$. Choosing the former option, the critical value $\omega_{c}=\omega_{c}(\gamma, \alpha)$ is the solution of the following equation

$$
\widetilde{F}\left(\omega_{c}\right)=F_{\alpha}(\gamma)+F_{\alpha}(\tilde{\gamma}) \quad \tilde{\gamma}=\pi-\left(\omega_{c}+\gamma\right)
$$

In figure 20 we show the surface which characterises the critical configurations, obtained by solving (7.5) numerically. Notice that the surface lies in the range $\alpha>\alpha_{c}$, as expected from the above considerations. The red solid curve in figure 20 corresponds to 


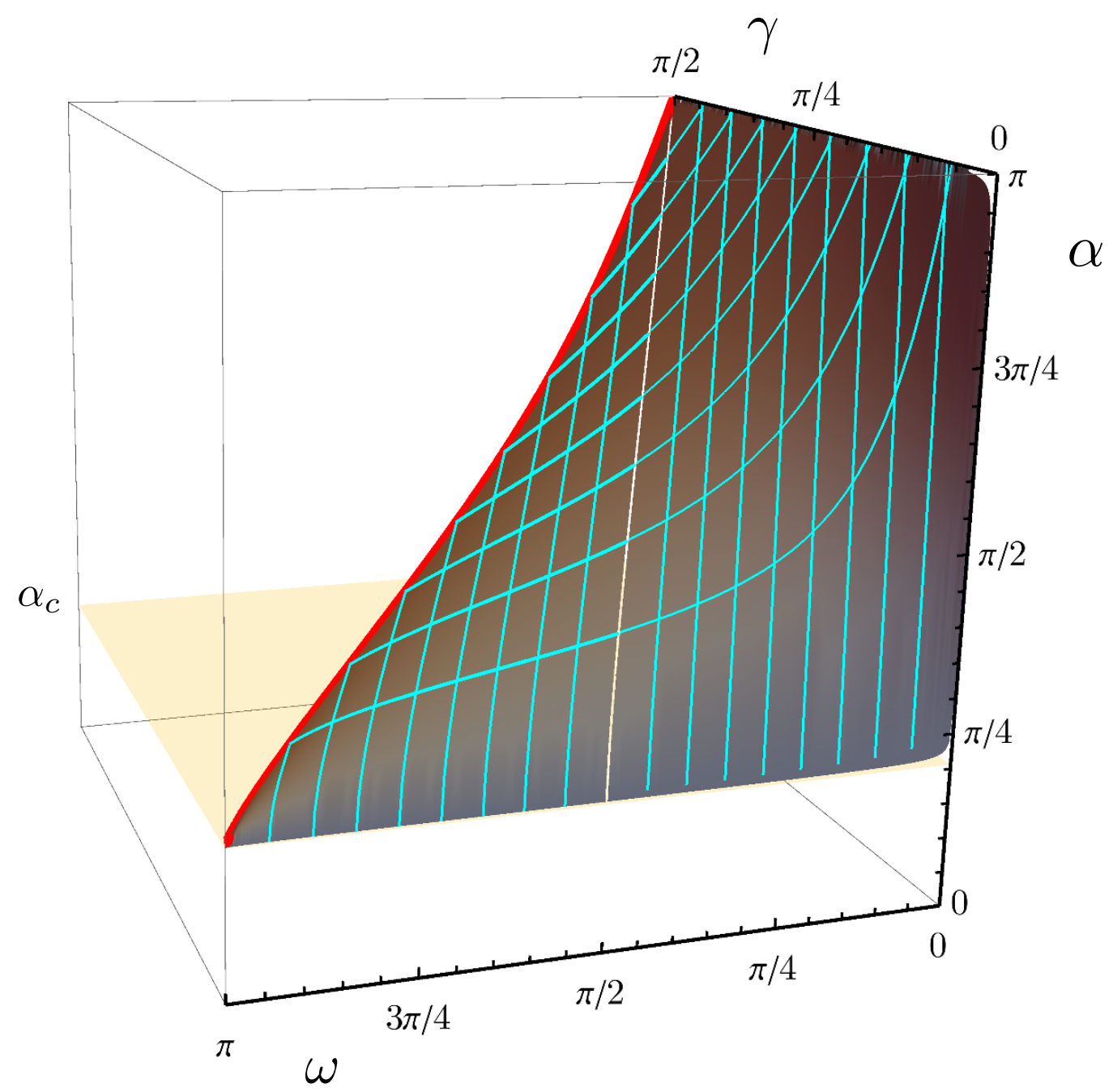

Figure 20. Infinite wedge with only the tip on the boundary: the surface described by the critical configurations, defined by (7.5) in the parameters space given by the angles $\omega, \gamma$ and $\alpha$. The yellow plane is $\alpha=\alpha_{c}$. The red curve corresponds to the symmetric configurations having $\tilde{\gamma}=\gamma$ (see figure 18).

the symmetric case $\gamma=\tilde{\gamma}$, namely to the curve in figure 18. Furthermore, the section at $\alpha=\pi / 2$ of the surface in figure 20 provides the critical configurations for the symmetric domains in a $\mathrm{CFT}_{3}$ whose coefficient of the logarithmic divergence of the corresponding holographic entanglement entropy is (3.7), which have been described in section 3.2 .

\section{Conclusions}

Understanding the role of the boundary conditions in the analysis of the entanglement entropy in $\mathrm{BCFT}_{3}$ is an interesting problem within the program of studying entanglement in quantum field theories.

Considering a $\mathrm{BCFT}_{3}$ with a flat boundary, in this manuscript we mainly focussed on the entanglement entropy of two dimensional domains $A$ in a constant time slice whose 
boundaries $\partial A$ intersect the boundary of the $\mathrm{BCFT}_{3}$. In particular we have studied the cases where the singular points of $\partial A$ belong also to the boundary of the $\mathrm{BCFT}_{3}$ (see e.g. the yellow region in the right panel of figure 1). The expansion of the entanglement entropy of these domains as the UV cutoff $\varepsilon \rightarrow 0$ contains also a logarithmic divergence whose coefficient encodes the characteristic features of the $\mathrm{BCFT}_{3}$ through some corner functions in a non trivial way.

In this manuscript we have studied the holographic corner functions in the $\mathrm{AdS}_{4} / \mathrm{BCFT}_{3}$ setup introduced in [63], where the gravitational spacetime is bounded by a surface $\widetilde{\mathcal{Q}}$ anchored to the boundary of the $\mathrm{BCFT}_{3}$, which is obtained by solving certain Neumann boundary conditions. In our simplified case where the boundary of the $\mathrm{BCFT}_{3}$ is flat, $\widetilde{\mathcal{Q}}$ on a constant time slice is given by a half plane $\mathcal{Q}$ characterised by its slope $\alpha \in(0, \pi)$.

The holographic entanglement entropy has been computed by employing the prescription (4.5), where the minimal area surface $\hat{\gamma}_{A}$ must be found among the surfaces $\gamma_{A}$ anchored to the entangling curve $\partial A \cap \partial B$. Since the curve $\gamma_{A} \cap \mathcal{Q}$ can vary and restrictions are not imposed on it, the minimisation of the area leads to the condition that $\hat{\gamma}_{A}$ is orthogonal to $\mathcal{Q}$ along the curve given by their intersection.

In this $\mathrm{AdS}_{4} / \mathrm{BCFT}_{3}$ setup, as preliminary simple cases we have computed the holographic entanglement entropy of infinite strips, both adjacent and parallel to the boundary (see also [73]), and of a half disk centered on the boundary.

Our main result is the analytic expression of the corner function $F_{\alpha}(\gamma)$ for an infinite wedge adjacent to the boundary, which is given by (6.5) and (6.10) in a parametric form (see figure 14 and figure 15). This result and the corner function of [27] lead to the analytic formula (7.1) for the corner function $\mathcal{F}_{\alpha}(\omega, \gamma)$, which corresponds to an infinite wedge having only its tip on the boundary.

Various checks have been done to test the analytic expressions of these two corner functions. The main one is the numerical analysis performed by employing Surface Evolver [76, 77], where minimal area surfaces corresponding to finite domains containing corners have been explicitly constructed in order to study the coefficient of the logarithmic divergence of their area. Further non trivial consistency checks have been considered by studying the limiting regimes $\gamma \rightarrow 0^{+}$and $\gamma \rightarrow \pi / 2$ of the corner function $F_{\alpha}(\gamma)$. In the limit $\gamma \rightarrow 0^{+}$the holographic entanglement entropy of the infinite strip adjacent to the boundary has been recovered, while taking the limit $\gamma \rightarrow \pi / 2$ we have obtained the coefficient of the logarithmic divergence in the holographic entanglement entropy of the half disk centered on the boundary, as expected.

We remark that interesting transitions have been observed in the analysis of the holographic entanglement entropy for the various domains. The main one occurs in the slope $\alpha$ at the critical value given by (5.6). This transition can be observed also through the behaviour of the corner function $F_{\alpha}(\gamma)$ in the regime $\gamma \rightarrow 0^{+}$. We have also studied the transitions occurring in the holographic entanglement entropy of an infinite strip parallel to the boundary and at finite distance from it (see figure 11) and of an infinite wedge with only the tip on the boundary (see figure 20). 
Some of the results mentioned above have been studied for a generic spacetime dimension.

An interesting outcome of our analysis is the relation (6.24) found in the context of the $\mathrm{AdS}_{4} / \mathrm{BCFT}_{3}$ correspondence defined in [63], which involves the coefficient $f_{\alpha}^{\prime \prime}(\pi / 2)$ obtained from the expansion of $F_{\alpha}(\gamma)$ as $\gamma \rightarrow \pi / 2$ and the coefficient $A_{T}$ characterising the behaviour of the one point function of the stress tensor $\left\langle T_{i j}\right\rangle$ close to the boundary (see (6.20)). In particular, (6.24) tells us that the ratio between these coefficients is independent of $\alpha$.

Let us conclude by mentioning some open problems for future studies.

An important conceptual issue to understand in the AdS/BCFT setup of [63] is the possible relation occurring between the geometrical parameter $\alpha$ and the space of the conformally invariant boundary conditions for the dual $\mathrm{BCFT}_{3}$. We find it important also to study different AdS/BCFT constructions [72-74]. Within these setups, it is also relevant to consider a dual $\mathrm{BCFT}_{3}$ with non flat boundaries, which are not related to the flat one through a conformal transformation.

An interesting issue that we find worth exploring is the possibility that the relation (6.24) holds for other models of $\mathrm{BCFT}_{3}$.

Finally, the extension of the analysis performed in this manuscript to higher dimensions, where different kinds of singular configurations occur, is certainly important to improve our understanding of the holographic entanglement entropy in AdS/BCFT.

\section{Acknowledgments}

It is our pleasure to thank Andrea Coser, Andrea Gambassi, Matthew Headrick, Esperanza Lopez and Mukund Rangamani for useful discussions. We are grateful in particular to Dmitri Fursaev, Rong-Xin Miao, Sergey Solodukhin and Tadashi Takayanagi for important comments or correspondence. We acknowledge in a special way Cristiano De Nobili for his crucial help with Surface Evolver. ET is grateful to the IFT, Madrid, and to the University of Florence for the warm hospitality during some stages of this work.

\section{A On the numerical analysis}

Our numerical analysis is based on Surface Evolver, a multipurpose optimisation software developed by Ken Brakke [76, 77]. This tool is employed here to find minimal area surfaces embedded in the three dimensional hyperbolic space $\mathbb{H}_{3}$, whose metric is (3.1). The constraints imposed on the minimal surfaces define the ones we are interested in.

In this manuscript we deal with two qualitative different situations, depending on the occurrence of the half plane $\mathcal{Q}$ defined by (4.3). For the corner functions in $\mathrm{AdS}_{4} / \mathrm{CFT}_{3}$ discussed in section 3 we employ the standard prescription (1.6) for the holographic entanglement entropy, which requires to construct the minimal surface $\hat{\gamma}_{A}$ anchored to $\partial A$ in the $z=$ 0 plane. Instead, to compute the holographic entanglement entropy in $\mathrm{AdS}_{4} / \mathrm{BCFT}_{3}$ discussed in section 4 , the minimal area surface $\hat{\gamma}_{A}$ belongs to the region of $\mathbb{H}_{3}$ defined by (4.4) and it must be anchored only to the entangling curve $\partial A \cap \partial B$ in the $z=0$ half plane. Thus, 
while in the former case $\partial \hat{\gamma}_{A}=\partial A$, in the latter one $\partial \hat{\gamma}_{A} \subset \partial A$ and it can happen that $\partial \hat{\gamma}_{A} \cap$ $\mathcal{Q} \neq \emptyset$. When $\partial \hat{\gamma}_{A} \cap \mathcal{Q} \neq \emptyset$, the minimisation procedure implemented by Surface Evolver leads to surfaces which are orthogonal to $\mathcal{Q}$ along $\hat{\gamma}_{A} \cap \mathcal{Q}$ in the final step of the evolution.

Surface Evolver constructs surfaces as unions of triangles; therefore a smooth surface is approximated by a surface made by triangles obtained through a particular evolution. The initial step of the optimisation procedure is a very simple surface, made by few triangles, which basically sets the topology. The initial surface evolves towards a configuration which is a local minimum of the area functional by both increasing the number of triangles and modifying the mesh in a proper way. For each step of the evolution, the software provides all the elements characterising the surface, like the coordinates of the vertices, the way to connect them, the normal vectors, the area of each triangle, the total number of triangles and the total area of the surface. We refer the interested reader also the appendix B of [60] for another discussion on the application of Surface Evolver to find minimal area surfaces in $\mathbb{H}_{3}$.

Since the area of a surface reaching the boundary at $z=0$ diverges, in our numerical analysis we have defined the entangling curve $\partial A \cap \partial B$ (which coincides with $\partial A$ for the domains considered in section 3 to study the corner functions in $\mathrm{AdS}_{4} / \mathrm{CFT}_{3}$ ) at $z=\varepsilon$ and not at $z=0$, as required in the prescription for the holographic entanglement entropy. This way to regularise the final result does not influence the coefficients of the diverging terms in the expansion of the area $\mathcal{A}\left[\hat{\gamma}_{\varepsilon}\right]$ as $\varepsilon \rightarrow 0^{+}[27]$.

Once the final entangling curve $\partial A \cap \partial B$ has been fixed at $z=\varepsilon$, let us denote by $\gamma_{\varepsilon}^{\mathrm{SE}}$ the triangulated surface constructed by Surface Evolver at a generic step of the evolution and by $\tilde{\mathcal{A}}\left[\gamma_{\varepsilon}^{\mathrm{SE}}\right]$ the corresponding numerical value for its area provided by the software. We denote by $\tilde{\gamma}_{\varepsilon}^{\mathrm{SE}}$ the final configuration of the evolution and by $\tilde{\mathcal{A}}\left[\tilde{\gamma}_{\varepsilon}^{\mathrm{SE}}\right]$ the corresponding area given by Surface Evolver. The final step of the evolution depends on the required level of approximation. In our analysis the typical value of the UV cutoff is $\varepsilon=0.03$, the area of the final surfaces is $O\left(10^{2}\right)$ (setting $L_{\mathrm{AdS}}=1$ ) and we have stopped the evolution once the value of the area was stable up to small variations of order $O\left(10^{-2}\right)$.

The evolution begins from a very simple trial surface and it develops through a number of steps which improves the triangulation of the surface towards configurations with smaller area. A way to improve the triangulation consists in moving the positions of the vertices without changing their total number according to a gradient descent method which decreases the total area of the surface. Another way is to refine the mesh of the surface by splitting each edge of a facet into two new edges and then connecting them. After a modification of this kind, a facet is partitioned into four new facets; therefore this step increases the total number of triangles.

The boundaries of the triangulated surfaces are treated differently during the evolution, depending on whether they belong to the half plane $\mathcal{Q}$ or to the section of the spacetime given by $z=\varepsilon$ (for the surfaces studied in section 3 only the latter situation occurs). The vertices on the entangling curve $\partial A \cap \partial B$ at $z=\varepsilon$ are kept fixed although their number increases during the refinements. Instead, the vertices of the curve $\partial \gamma_{\varepsilon}^{\mathrm{SE}} \cap \mathcal{Q}$ can move freely on $\mathcal{Q}$ during the evolution.

In the top part of figure 21 we show some steps of an evolution made by Surface Evolver towards the minimal area surface anchored to the entangling curve given by the red line 

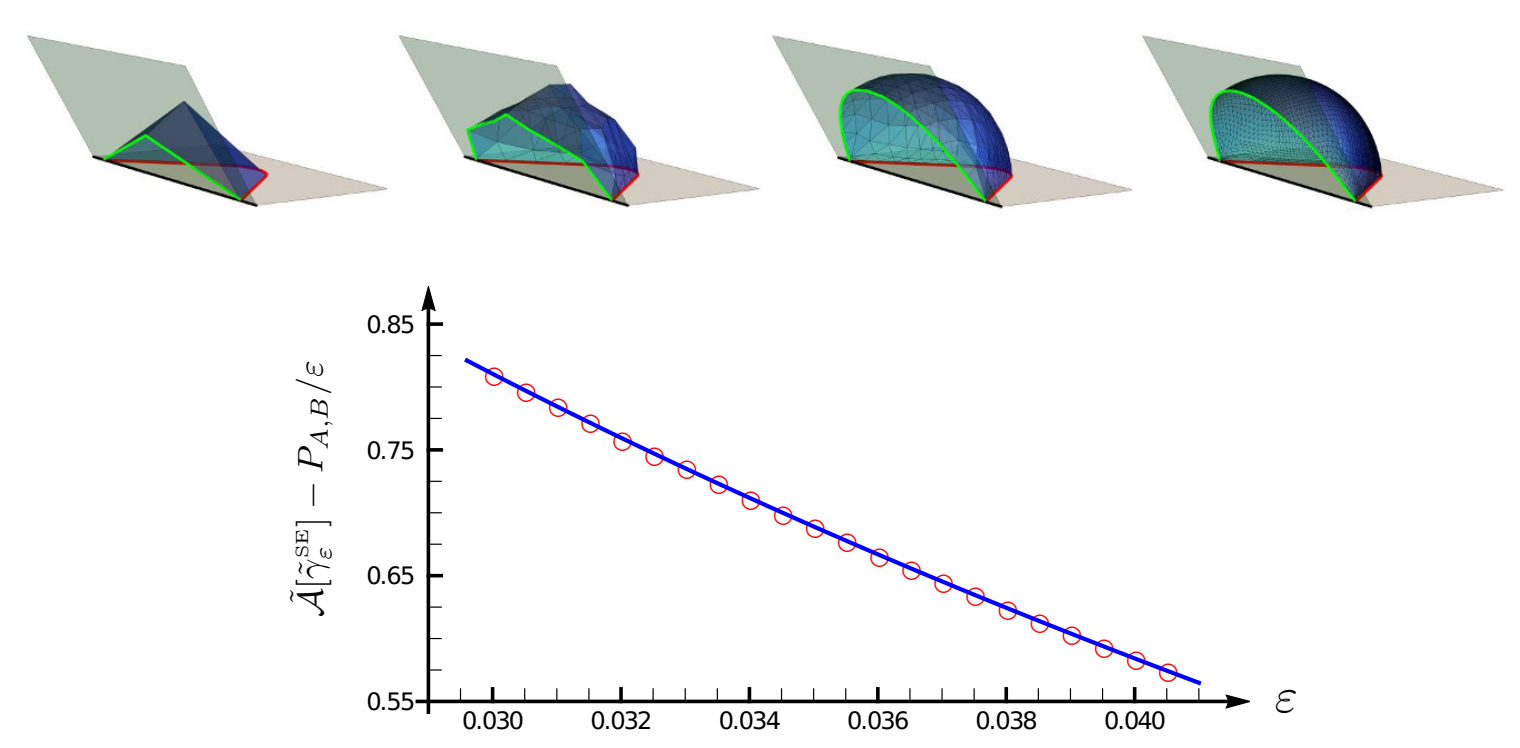

Figure 21. An example of the numerical analysis of the corner functions based on Surface Evolver. Top: some stages of an evolution towards the minimal area surface anchored to the entangling curve given by the red line in the $z=0$ half plane, which has $\gamma=\pi / 4$ and $L=2.5$ (see also figure 13). Here $\alpha=\pi / 3$. Bottom: numerical data corresponding to the evolutions shown in the top panel for different values of $\varepsilon$. Fitting this data as discussed in section A, one finds the numerical value for the corner function to compare with the corresponding one obtained from the analytic expression $F_{\alpha}(\gamma)$ given by $(6.5)$ and $(6.10)$.

in the $z=0$ plane (see also figure 13). In this example $\partial \gamma_{\varepsilon}^{\mathrm{SE}} \cap \mathcal{Q} \neq \emptyset$. The initial step of the evolution is a trial surface made by 6 facets while the last step shown in the figure is a triangulated surface with 6144 facets.

The coefficient of the logarithmic divergence in the expansion of the area as $\varepsilon \rightarrow 0^{+}$ has been extracted as follows. Once the final step $\tilde{\gamma}_{\varepsilon}^{\mathrm{SE}}$ of the evolution corresponding to a given entangling curve at $z=\varepsilon$ is reached, one subtracts to $\tilde{\mathcal{A}}\left[\tilde{\gamma}_{\varepsilon}^{\mathrm{SE}}\right]$ the area law term, which is given by either $P_{A} / \varepsilon$ or $P_{A, B} / \varepsilon$. By repeating this analysis for various small values of $\varepsilon$, a list of numerical values is obtained. Fitting these data points through the function $a \log \varepsilon+b+c \varepsilon$, one finds the best fit for the parameters $a, b$ and $c$. The value of $a$ is the numerical result for the coefficient of the logarithmic divergence that we have compared against the corresponding theoretical prediction. In the bottom part of figure 21 we show an example of this procedure which corresponds to the domain $A$ identified by the red curve in the top part of the same figure.

As a final technical remark, let us observe that, whenever $\tilde{\gamma}_{\varepsilon}^{\mathrm{SE}} \cap \mathcal{Q} \neq \emptyset$ the numerical analysis gets worse as the angle $\gamma$ decreases because of the formation of spikes at the tips of the corners. This explains why we did not obtain reliable results for small values of $\gamma$ in figure 14. The occurrence of unexpected spikes depends on $\alpha$ and it is observed for larger values of $\gamma$ as $\alpha$ decreases (see the lowest curve in figure 14). 


\section{B On the half disk centered on the boundary}

In this appendix we report the computation of the area $\mathcal{A}\left[\hat{\gamma}_{\varepsilon}\right]$, which provides the holographic entanglement entropy of half disk of radius $R$ centered on the boundary, according to the prescription (4.5). The main result derived here is (5.1), which is discussed in section 5.1.

Given the half disk $A=\left\{(x, y) \in \mathbb{R}^{2} \mid x^{2}+y^{2} \leqslant R^{2}, x \geqslant 0\right\}$, which is centered on the boundary $x=0$, the entangling curve $\partial A \cap \partial B$ is $\left\{(x, y) \in \mathbb{R}^{2} \mid x^{2}+y^{2}=R^{2}, x \geqslant 0\right\}$. In section 5.1 we have discussed for this domain $\hat{\gamma}_{A \text {,aux }}$ is the hemisphere $x^{2}+y^{2}+z^{2}=R^{2}$ in $\mathbb{H}_{3}$ and that $\hat{\gamma}_{A}$ is just the part of $\hat{\gamma}_{A \text {,aux }}$ identified by the constraint (4.4). In figure 8 , the minimal surface $\hat{\gamma}_{A}$ is shown in a case having $\alpha<\pi / 2$ and in a case where $\alpha>\pi / 2$.

The holographic entanglement entropy is obtained by evaluating the area $\mathcal{A}\left[\hat{\gamma}_{\varepsilon}\right]$ of the surface $\hat{\gamma}_{A} \cap\{z \geqslant \varepsilon\}$, which is the part of $\hat{\gamma}_{A}$ above the yellow line in figure 8. This area can be written as follows

$$
\frac{\mathcal{A}\left[\hat{\gamma}_{\varepsilon}\right]}{L_{\mathrm{AdS}}^{2}}= \begin{cases}\mathcal{A}_{\perp}+\mathcal{A}_{\llcorner} & 0<\alpha \leqslant \pi / 2 \\ \mathcal{A}_{\perp}-\mathcal{A}_{\llcorner} & \pi / 2 \leqslant \alpha<\pi\end{cases}
$$

where $\mathcal{A}_{\perp}$ is the area of the half hemisphere restricted to $z \geqslant \varepsilon$ with $x \geqslant 0$ and $\mathcal{A}_{\angle} \geqslant 0$ is the area of the part of the hemisphere restricted to $z \geqslant \varepsilon$ enclosed between the vertical half plane $x=0$ and the half plane $\mathcal{Q}$. Notice that, in the right panel of figure 8 , the area $\mathcal{A}_{\angle}$ corresponds to the shaded part of $\hat{\gamma}_{A \text {,aux }}$.

The area $\mathcal{A}_{\perp}$ can be easily computed by adopting the usual spherical coordinates $(\theta, \phi)$, where $\theta=0$ is the positive $z$ semi-axis and $\phi=0$ is the positive $y$ semi-axis. The change of coordinates between these polar coordinates and the Cartesian coordinates reads

$$
z=R \cos \theta \quad x=R \sin \theta \sin \phi \quad y=R \sin \theta \cos \phi
$$

In terms of the polar coordinates $(\theta, \phi)$, the induced metric on $\hat{\gamma}_{A}$ from $\mathbb{H}_{3}$ is given by

$$
\left.d s^{2}\right|_{\hat{\gamma}_{A}}=\frac{L_{\text {AdS }}^{2}}{(\cos \theta)^{2}}\left(d \theta^{2}+(\sin \theta)^{2} d \phi^{2}\right)
$$

By employing this metric, for $\mathcal{A}_{\perp}$ we find

$$
\mathcal{A}_{\perp}=\int_{0}^{\theta_{\varepsilon}} d \theta \int_{0}^{\pi} d \phi \frac{\sin \theta}{(\cos \theta)^{2}}=\left.\frac{\pi}{\cos \theta}\right|_{0} ^{\theta_{\varepsilon}}=\frac{\pi R}{\varepsilon}-\pi
$$

where the condition defining $\theta_{\varepsilon}$ is $\varepsilon=R \cos \theta_{\varepsilon}$.

In order to compute $\mathcal{A}_{L}$, let us parameterise the hemisphere by employing spherical coordinates $(\theta, \phi)$, where $\theta=0$ is the positive $y$ semi-axis and $\phi=0$ is the positive $z$ semi-axis. Now the change of coordinates is

$$
z=R \sin \theta \cos \phi \quad x=-R \sin \theta \sin \phi \quad y=R \cos \theta
$$

The induced metric on $\hat{\gamma}_{A}$ from $\mathbb{H}_{3}$ in terms of these polar coordinates is

$$
\left.d s^{2}\right|_{\hat{\gamma}_{A}}=\frac{L_{\mathrm{AdS}}^{2}}{(\sin \theta)^{2}(\cos \phi)^{2}}\left(d \theta^{2}+(\sin \theta)^{2} d \phi^{2}\right)
$$


From the first expression in (B.5) we obtain $\varepsilon=R \sin \theta_{\varepsilon} \cos \phi$, which relates the UV cutoff $\varepsilon$ to the cutoff $\theta_{\varepsilon}$ of the angular variable. This relation leads to $\sin \left(\theta_{\varepsilon} / 2\right)=\varepsilon[1+$ $\left.O\left(\varepsilon^{2}\right)\right] /(2 R \cos \phi)$.

When $\alpha \in(0, \pi / 2)$, the area $\mathcal{A}_{\angle}$ is given by the following integral

$$
\begin{aligned}
\mathcal{A}_{L} & =2 \int_{0}^{\pi / 2-\alpha} d \phi \int_{\theta_{\varepsilon}}^{\pi / 2} d \theta \frac{1}{(\cos \phi)^{2} \sin \theta}=-2 \int_{0}^{\pi / 2-\alpha} d \phi \frac{\log \left(\tan \theta_{\varepsilon} / 2\right)}{(\cos \phi)^{2}} \\
& =2 \int_{0}^{\pi / 2-\alpha} d \phi \frac{\frac{1}{2} \log \left(1-\left[\sin \left(\theta_{\varepsilon} / 2\right)\right]^{2}\right)-\log \left[\sin \left(\theta_{\varepsilon} / 2\right)\right]}{(\cos \phi)^{2}} \\
& =2 \int_{0}^{\pi / 2-\alpha} d \phi \frac{1}{(\cos \phi)^{2}}\left(\frac{1}{2} \log \left(1-\varepsilon^{2} /[2 R \cos \phi]^{2}\right)-\log (\varepsilon / R)+\log (2 \cos \phi)\right)+O\left(\varepsilon^{2}\right) \\
& =2(\cot \alpha) \log (R / \varepsilon)+O(1)
\end{aligned}
$$

where in (B.7) the relation between $\theta_{\varepsilon}$ and $\varepsilon$ has been employed and the $O\left(\varepsilon^{2}\right)$ terms have been neglected. The $O(1)$ term in (B.7) can be found explicitly, but we do not report it here because we are interested only in the logarithmic divergence. When $\alpha \in(\pi / 2, \pi)$, being $\mathcal{A}_{\angle}>0$, the resulting integral for $\mathcal{A}_{\angle}$ is like (B.7), except for the domain of integration for the integral in $\phi$, which is $(0, \alpha-\pi / 2)$.

Summarising, the term $\mathcal{A}_{\angle}$ provides the following logarithmic divergence

$$
\mathcal{A}_{L}=\left\{\begin{array}{cc}
2(\cot \alpha) \log (R / \varepsilon)+O(1) & 0<\alpha \leqslant \pi / 2 \\
-2(\cot \alpha) \log (R / \varepsilon)+O(1) & \pi / 2 \leqslant \alpha<\pi
\end{array}\right.
$$

Finally, by plugging (B.4) and (B.8) into (B.1), we obtain the area $\mathcal{A}\left[\hat{\gamma}_{\varepsilon}\right]$ given by (5.1), which is the main result of this appendix.

Let us stress that the holographic entanglement entropy for this domain provides the corner function $F_{\alpha}(\pi / 2)$ for the special value $\gamma=\pi / 2$ and for any $\alpha \in(0, \pi)$. This is an important benchmark for the analytic expression of the corner function $F_{\alpha}(\gamma)$ presented in section 6 , whose derivation is described in the appendix F.

\section{Infinite strip adjacent to the boundary in generic dimension}

In this appendix we study the holographic entanglement entropy for the $d$ dimensional infinite strip of width $\ell$ adjacent to the boundary. The main results of this analysis specialised to $d=2$ have been reported in section 5.2.

Given a constant time slice of a $\mathrm{BCFT}_{d+1}$, defined by $x \geqslant 0$ in proper Cartesian coordinates, let us consider the following spatial domain

$$
A=\left\{\left(x, y_{1}, \ldots, y_{d-1}\right) \mid 0 \leqslant x \leqslant \ell, 0 \leqslant y_{i} \leqslant L_{\|}\right\} \quad L_{\|} \gg \ell \gg \varepsilon
$$

The invariance under translations along the $y_{i}$-axis (in a strict sense, this requires $L_{\|} \rightarrow$ $+\infty$ ) allows us to assume that the minimal surface $\hat{\gamma}_{A}$ is characterised by its profile obtained by sectioning $\hat{\gamma}_{A}$ through an hyperplane defined by $y_{i}=$ const. The profile of $\hat{\gamma}_{A}$ is given by either $x=\ell$ or by a non trivial curve $z=z(x)$. Focussing on the latter case, let us denote 
by $P_{*}=\left(x_{*}, z_{*}\right)$ the intersection between the curve $z(x)$ and the section at $y_{i}=$ const of the half hyperplane $\mathcal{Q}$, which is a half line given by (4.3). The coordinates of $P_{*}$ are constrained by imposing that $P_{*} \in \mathcal{Q}$ and this condition gives

$$
x_{*}=-z_{*} \cot \alpha
$$

where we recall that $\alpha \in(0, \pi)$. Since the curve $z(x)$ characterising the extremal surface intersects orthogonally the section at constant $y_{i}=$ const of the half hyperplane $\mathcal{Q}$, it is not difficult to realise that $z^{\prime}\left(x_{*}\right)=\cot \alpha$.

The profile $z(x)$ can be obtained by finding the extrema of the area functional among the surfaces $\gamma_{A}$ anchored to the edge $x=\ell$ of the strip (C.1) which are invariant under translations along the $y_{i}$ directions and intersect $\mathcal{Q}$ orthogonally.

Given a surface $\gamma_{A}$ characterised by $z(x)$, by writing the metric induced on $\gamma_{A}$ from the background (4.2), one obtains the following area functional

$$
\mathcal{A}\left[\gamma_{A}\right]=L_{\mathrm{AdS}}^{d} L_{\|}^{d-1} \int_{x_{*}}^{\ell} \frac{\sqrt{1+\left(z^{\prime}\right)^{2}}}{z^{d}} d x
$$

Since the integrand does not depend on $x$ explicitly, we can find the extremal surface $\hat{\gamma}_{A}$ by employing the fact that the first integral of motion is constant. For the functional (C.3) this condition tells us that $z^{d} \sqrt{1+\left(z^{\prime}\right)^{2}}$ is independent of $x$; therefore we can evaluate it at any point on the extremal surface described by $z(x)$. By choosing the point $\left(x_{*}, z_{*}\right)$, where $z^{\prime}\left(x_{*}\right)=\cot \alpha$, the equation imposing the constancy of the first integral of motion reads

$$
z^{d} \sqrt{1+\left(z^{\prime}\right)^{2}}=\frac{z_{*}^{d}}{\sin \alpha}
$$

In order to solve (C.4), we find it convenient to introduce the following parameterisation

$$
z(\theta)=\frac{z_{*}}{(\sin \alpha)^{1 / d}}(\sin \theta)^{1 / d} \quad 0 \leqslant \theta \leqslant \pi-\alpha
$$

which respects the boundary conditions $z(\pi-\alpha)=z_{*}$ and $z(0)=0$.

Plugging (C.5) into the square of (C.4), one gets $\left(\frac{d z}{d x}\right)^{2}=(\cot \theta)^{2}$, which gives $x^{\prime}(\theta)^{2}=$ $z^{\prime}(\theta)^{2}(\tan \theta)^{2}$. Then, by employing (C.5) into the latter differential equation, we obtain

$$
x^{\prime}(\theta)=-\frac{z_{*}}{d(\sin \alpha)^{1 / d}}(\sin \theta)^{1 / d}
$$

where the physical condition that $x(\theta)$ decreases for increasing values of $\theta$ has been imposed.

The relation $\left(\frac{d z}{d x}\right)^{2}=(\cot \theta)^{2}$ and (C.5) leads to the geometrical meaning of the angle $\theta$ : it is the angle between the outgoing vector normal to the curve given by $P_{\theta}$ and the $x$ semi-axis with $x \geqslant 0$. Thus, from (C.5) we have that $\theta=\pi / 2$ corresponds to the point of the curve $z(x)$ having the maximum value $z_{\max }=z_{*} /(\sin \alpha)^{1 / d}$.

By integrating (C.6) with the initial condition $x(0)=\ell$, we find

$$
\begin{aligned}
x(\theta) & =\ell-\frac{z_{*}}{d(\sin \alpha)^{1 / d}} \int_{0}^{\theta}(\sin \tilde{\theta})^{1 / d} d \tilde{\theta} \\
& =\ell-\frac{z_{*}}{(\sin \alpha)^{1 / d}}\left[\frac{\sqrt{\pi} \Gamma\left(\frac{d+1}{2 d}\right)}{\Gamma\left(\frac{1}{2 d}\right)}-\frac{\cos \theta}{d}{ }_{2} F_{1}\left(\frac{d-1}{2 d}, \frac{1}{2} ; \frac{3}{2} ;(\cos \theta)^{2}\right)\right]
\end{aligned}
$$




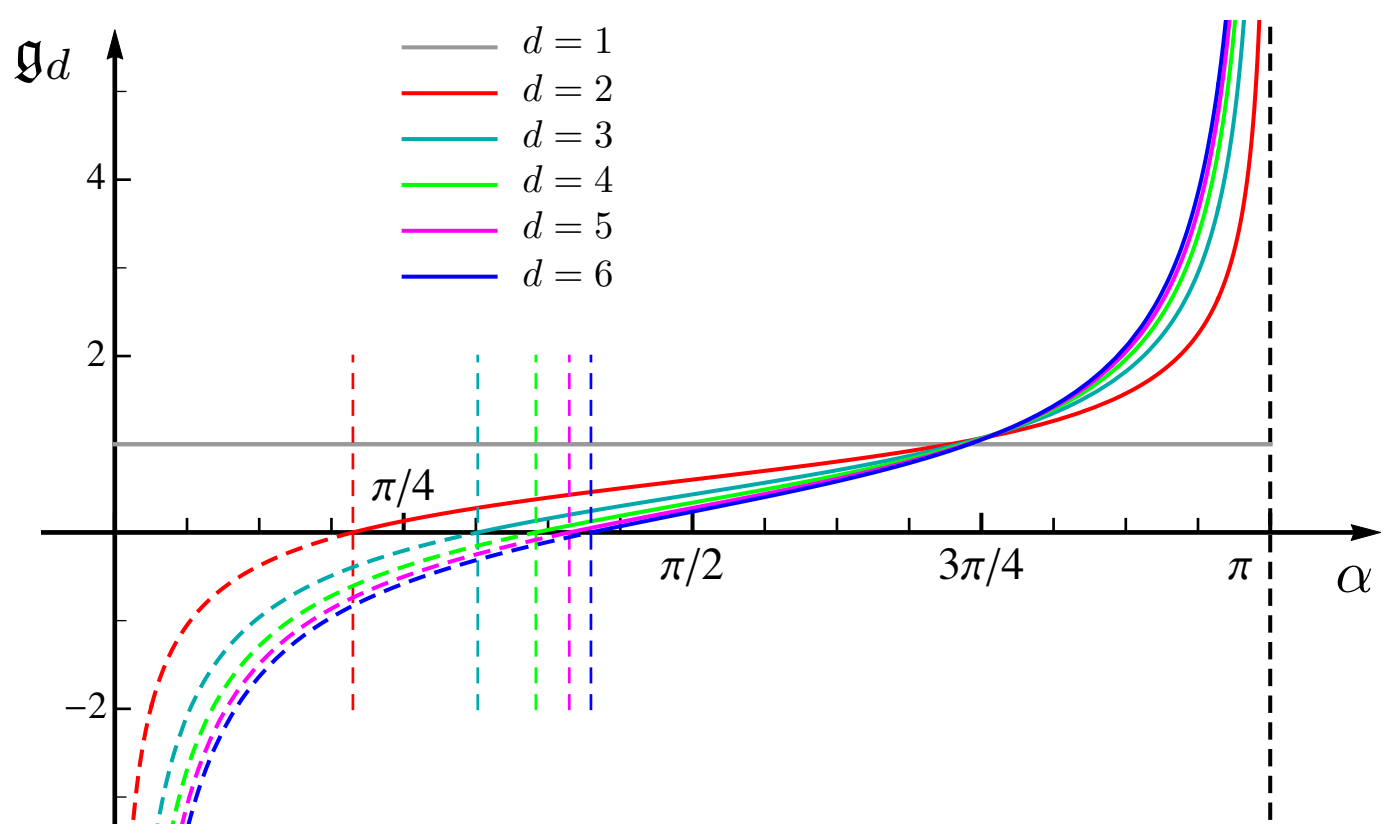

Figure 22. The function $\mathfrak{g}_{d}(\alpha)$ defined in (C.11) for some values of $d$. For a given $d$, the critical value $\alpha_{c}(d)$ is the unique zero of $\mathfrak{g}_{d}(\alpha)$ (see (C.13)) and it has been highlighted through a vertical dashed segment having the same colour of the corresponding curve $\mathfrak{g}_{d}(\alpha)$.

The expressions (C.5) and (C.8) depend on the coordinate $z_{*}$ of the point $P_{*}$. We can relate $z_{*}$ to the width $\ell$ of the strip (C.1) by imposing that (C.8) satisfies the consistency condition $x(\pi-\alpha)=x_{*}$, where $x_{*}$ can be obtained from (C.2). This gives

$$
\ell-\frac{z_{*}}{(\sin \alpha)^{1 / d}}\left[\frac{\sqrt{\pi} \Gamma\left(\frac{d+1}{2 d}\right)}{\Gamma\left(\frac{1}{2 d}\right)}+\frac{\cos \alpha}{d}{ }_{2} F_{1}\left(\frac{d-1}{2 d}, \frac{1}{2} ; \frac{3}{2} ;(\cos \alpha)^{2}\right)\right]=-z_{*} \cot \alpha
$$

which leads to the following relation

$$
z_{*}=\frac{(\sin \alpha)^{1 / d}}{\mathfrak{g}_{d}(\alpha)} \ell
$$

where we have introduced

$$
\mathfrak{g}_{d}(\alpha) \equiv \frac{\sqrt{\pi} \Gamma\left(\frac{d+1}{2 d}\right)}{\Gamma\left(\frac{1}{2 d}\right)}+\frac{\cos \alpha}{d}{ }_{2} F_{1}\left(\frac{d-1}{2 d}, \frac{1}{2} ; \frac{3}{2} ;(\cos \alpha)^{2}\right)-(\sin \alpha)^{1 / d} \cot \alpha
$$

We remark that $z_{*}>0$, therefore (C.10) is well defined only when $\mathfrak{g}_{d}(\alpha)>0$, being $\alpha \in$ $(0, \pi)$. For $d=2$, which is the case considered through the main text, the function (C.11) becomes the function $\mathfrak{g}(\alpha) \equiv \mathfrak{g}_{2}(\alpha)$ in (5.4).

The first derivative of $\mathfrak{g}_{d}(\alpha)$ with respect to $\alpha$ is very simple

$$
\partial_{\alpha} \mathfrak{g}_{d}(\alpha)=\left(1-\frac{1}{d}\right)(\sin \alpha)^{1 / d-2}
$$

This expression tells us that $\mathfrak{g}_{1}(\alpha)$ is constant and, in particular, one finds $\mathfrak{g}_{1}(\alpha)=1$ identically. When $d>1$, we have that $\mathfrak{g}_{d}^{\prime}(\alpha)>0$ for $\alpha \in(0, \pi)$. Moreover, $\mathfrak{g}_{d}(\alpha)=$ 


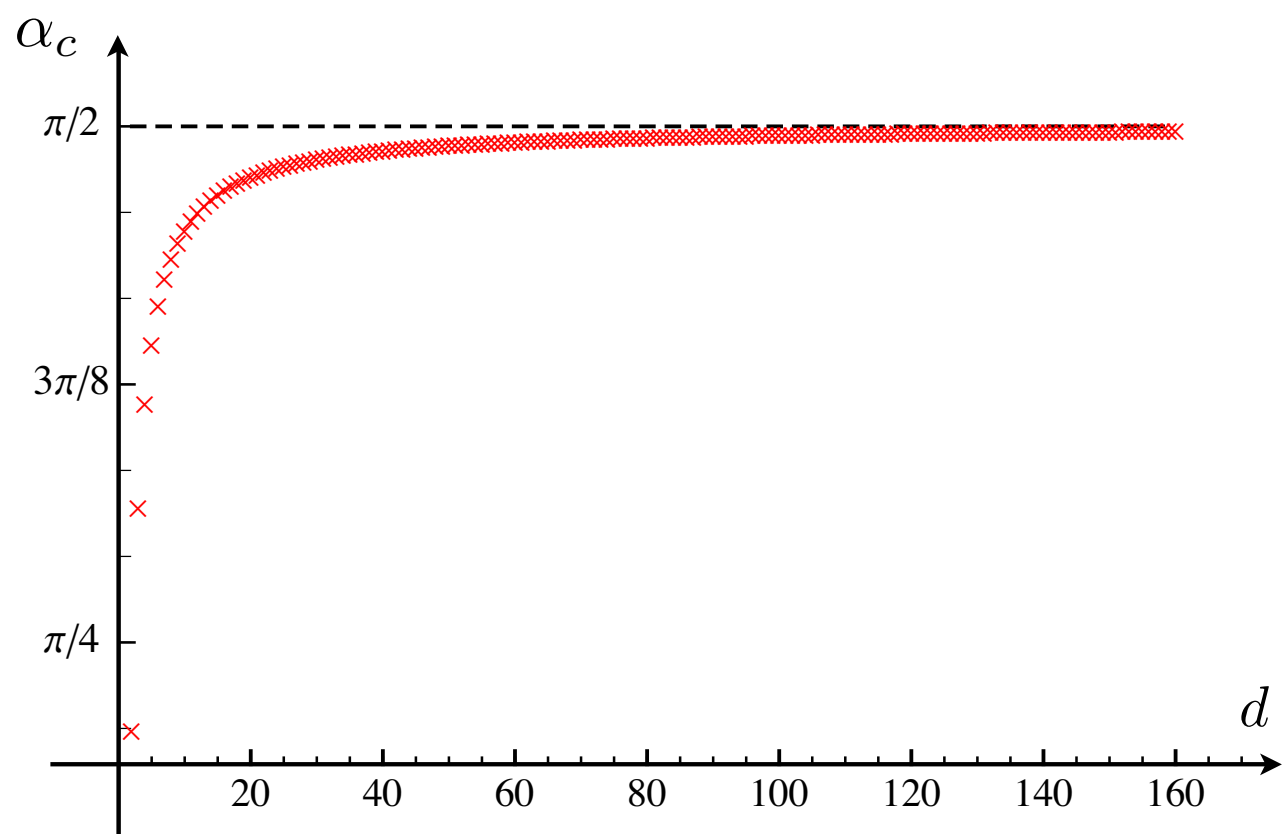

Figure 23. The critical slope $\alpha_{c}(d)$ of the half plane $\mathcal{Q}$ as function of the dimensionality parameter $d \geqslant 2$. These points have been found by solving (C.13). The value $\alpha_{c}(2)$ is given by (5.6). We find that $\alpha_{c}(d) \rightarrow \pi / 2$ as $d \rightarrow+\infty$.

$-1 / \alpha^{1-1 / d}+O(1)$ as $\alpha \rightarrow 0^{+}$and $\mathfrak{g}_{d}(\alpha)=1 /(\pi-\alpha)^{1-1 / d}+o(1)$ as $\alpha \rightarrow \pi^{-}$. These observations allow to conclude that (C.11) has a unique zero $\alpha=\alpha_{c}$ for $d>1$, namely

$$
\mathfrak{g}_{d}\left(\alpha_{c}\right)=0
$$

Since $z_{*}>0$ in (C.10), the condition (C.13) defines a critical value $\alpha_{c}(d)$ for the slope of $\mathcal{Q}$. Indeed, (C.10) is well defined only for $\alpha \in\left(\alpha_{c}, \pi\right)$. Moreover, from (C.10) and (C.13) we have that $z_{*} \rightarrow+\infty$ when $\alpha \rightarrow \alpha_{c}^{+}$. These observations allow us to conclude that for $\alpha \in\left(0, \alpha_{c}\right]$ the solution which intersects orthogonally the half hyperplane $\mathcal{Q}$ at a finite value of $z_{*}$ does not exist; therefore $\hat{\gamma}_{A}$ is the vertical half hyperplane $x=\ell$ in this range of $\alpha$.

We remark that $\alpha_{c} \leqslant \pi / 2$. Indeed, for $\alpha>\pi / 2$ it is straightforward to observe that the vertical half hyperplane $x=\ell$ is excluded because it does not intersect orthogonally the half hyperplane $\mathcal{Q}$.

We find it worth considering the limit $d \rightarrow+\infty$ of (C.11). In this regime only the last term gives a non vanishing contribution and, in particular, we have $\mathfrak{g}_{d}(\alpha) \rightarrow-\cot \alpha$, meaning that $\alpha_{c}(d) \rightarrow \pi / 2$. Thus, $\alpha_{c}$ tends to its natural upper bound for large $d$.

In figure 22 the function $\mathfrak{g}_{d}(\alpha)$ is shown for $1 \leqslant d \leqslant 6$. The corresponding critical values $\alpha_{c}(d)$ for $d \leqslant 2$ are highlighted through vertical dashed lines. The value of $\alpha_{c}(d=3)$ has been found also in [73]. In figure 23 we provide the critical slope $\alpha_{c}(d)$ as function of the dimensionality parameter $d$. 
The profile $z(x)$ of the extremal solution intersecting $\mathcal{Q}$ orthogonally at a finite value $z_{*}$ can be found by plugging (C.10) into (C.5) and (C.8). The result reads

$$
(x(\theta), z(\theta))=\frac{\ell}{\mathfrak{g}_{d}(\alpha)}\left(\frac{\cos \theta}{d}{ }_{2} F_{1}\left(\frac{d-1}{2 d}, \frac{1}{2} ; \frac{3}{2} ;(\cos \theta)^{2}\right)-\frac{\sqrt{\pi} \Gamma\left(\frac{d+1}{2 d}\right)}{\Gamma\left(\frac{1}{2 d}\right)}+\mathfrak{g}_{d}(\alpha),(\sin \theta)^{1 / d}\right)
$$

It is not difficult to check that this profile satisfies the required boundary conditions. Indeed for $\theta=0$ and $\theta=\pi-\alpha$ we find $P_{0}=(\ell, 0)$ and $P_{*}=z_{*}(-\cot \alpha, 1)$ respectively, being $z_{*}$ given by (C.10). The expression of (C.14) specialised to $d=2$ has been reported in (5.7).

An interesting point of the curve $z(x)$ is the one where $z^{\prime}(x)$ vanishes. Denoting its coordinates by $P_{\max }=\left(x_{\max }, z_{\max }\right)$, we have that $z^{\prime}\left(x_{\max }\right)=0$. From the latter condition and (C.4) one finds a relation between $P_{\max }$ and $P_{*}$ given by

$$
z_{\max }=\frac{z_{*}}{(\sin \alpha)^{1 / d}}=\frac{\ell}{\mathfrak{g}_{d}(\alpha)}
$$

The first equality can be obtained also from (C.5) for $\theta=\pi / 2$, as remarked above, while in the last step (C.10) has been used. Notice that for $0<\alpha<\pi / 2$ we have that $z_{\max }>z_{*}$, being $\theta-\alpha \neq \pi / 2$. Instead, $P_{\max }=P_{*}$ when $\alpha=\pi / 2$, while $P_{\max }$ does not exist when $\alpha>\pi / 2$. These features can be observed in figure 9 for the case $d=2$.

We find it worth remarking that the minimal surface $\hat{\gamma}_{A}$ characterised by (C.14) is part of an auxiliary surface $\hat{\gamma}_{A \text {,aux }}$ which has minimal area in the hyperbolic space $\mathbb{H}_{d+1}=$ $\left.A d S_{d+2}\right|_{t=\text { const }}$ and which is anchored to an infinite strip $A_{\text {aux }}$ of width $\ell_{\text {aux }}$ belonging to the boundary $z=0$ of $\mathbb{H}_{d+1}$. The auxiliary infinite strip $A_{\text {aux }}$ includes $A$ and it shares with $A$ the edge at $x=\ell$. The minimal surface $\hat{\gamma}_{A \text {,aux }}$ has been computed in $[5,6]$.

By employing the results of $[5,6]$ and imposing that (C.15) is also the largest value assumed by the coordinate $z$ for the points of $\hat{\gamma}_{A \text {,aux }}$, we find that

$$
\ell_{\mathrm{aux}}=2 \frac{\sqrt{\pi} \Gamma\left(\frac{d+1}{2 d}\right)}{\Gamma\left(\frac{1}{2 d}\right) \mathfrak{g}_{d}(\alpha)} \ell
$$

In particular, $\ell_{\text {aux }}$ depends on $\alpha$. As consistency check of (C.16), we observe that $\ell_{\text {aux }}=$ $\ell-x(\pi)$, where $x(\theta)$ has been written in (C.14).

In order to evaluate the area for $z \geqslant \varepsilon$ of the extremal surface characterised by the profile (C.14), let us compute the metric induced on this surface by the background metric of $\mathbb{H}_{d+1}$. By setting $t=$ const into (4.2) and employing the relation $x^{\prime}(\theta)^{2}=z^{\prime}(\theta)^{2}(\tan \theta)^{2}$ derived above (see the text below (C.5)), we find that the induced metric reads

$$
\begin{aligned}
\left.d s^{2}\right|_{\hat{\gamma}_{A}} & =\frac{L_{\mathrm{AdS}}^{2}}{z(\theta)^{2}}\left[\frac{z^{\prime}(\theta)^{2}}{(\cos \theta)^{2}} d \theta^{2}+d \vec{y}^{2}\right] \\
& =\frac{L_{\mathrm{AdS}}^{2}(\sin \alpha)^{2 / d}}{z_{*}^{2}(\sin \theta)^{2 / d}}\left[\frac{z_{*}^{2}}{d^{2}(\sin \alpha)^{2 / d}(\sin \theta)^{2(1-1 / d)}} d \theta^{2}+d \vec{y}^{2}\right]
\end{aligned}
$$

where $d \vec{y}^{2}=\sum_{j=1}^{d-1} d y_{j}^{2}$ and (C.5) have been used to obtain the last expression. 
Let us focus on the cases with $d>1$ first. From (C.18), for the area of $\hat{\gamma}_{\varepsilon}$ we find

$$
\begin{aligned}
\frac{\mathcal{A}\left[\hat{\gamma}_{\varepsilon}\right]}{L_{\mathrm{AdS}}^{d}} & =\frac{(\sin \alpha)^{1-1 / d}}{d z_{*}^{d-1}} \int_{0}^{L_{\|}} d y_{1} \ldots d y_{d-1} \int_{\theta_{\varepsilon}}^{\pi-\alpha} \frac{d \theta}{(\sin \theta)^{2-1 / d}} \\
& =\left.\frac{(\sin \alpha)^{1-1 / d}}{d z_{*}^{d-1}} L_{\|}^{d-1}\left[{ }_{2} F_{1}\left(\frac{3 d-1}{2 d}, \frac{1}{2} ; \frac{3}{2} ;(\cos \theta)^{2}\right) \cos \theta\right]\right|_{\pi-\alpha} ^{\theta_{\varepsilon}}
\end{aligned}
$$

where the cutoff $\theta_{\varepsilon}$ is defined by imposing that $z\left(\theta_{\varepsilon}\right)=\varepsilon$, being $z(\theta)$ the expression in (C.5). This gives $\theta_{\varepsilon}=\arcsin \left(\varepsilon^{d} \sin \alpha / z_{*}^{d}\right)$.

Taking the limit $\varepsilon \rightarrow 0^{+}$in (C.20) and neglecting terms which vanish in this limit, we find

$$
\begin{aligned}
\frac{\mathcal{A}\left[\hat{\gamma}_{\varepsilon}\right]}{L_{\mathrm{AdS}}^{d}}=L_{\|}^{d-1}\{ & \frac{1}{(d-1) \varepsilon^{d-1}} \\
& \left.\quad-\frac{(\sin \alpha)^{1-1 / d}}{z_{*}^{d-1}}\left[\frac{\sqrt{\pi} \Gamma\left(\frac{d+1}{2 d}\right)}{(d-1) \Gamma\left(\frac{1}{2 d}\right)}-\frac{\cos \alpha}{d}{ }_{2} F_{1}\left(\frac{3 d-1}{2 d}, \frac{1}{2} ; \frac{3}{2} ;(\cos \alpha)^{2}\right)\right]\right\}
\end{aligned}
$$

We remark that the divergent part of the area $\mathcal{A}\left[\hat{\gamma}_{\varepsilon}\right]$ is due to the area term only.

The above analysis extends smoothly to the whole range of $\alpha \in(0, \pi)$ the results of [73] for the infinite strip adjacent to the boundary, which hold for $\alpha \in(0, \pi / 2]$.

The finite term in (C.21) can be written in an insightful form by considering the following identity [99]

$$
[(c-b) x-a]_{2} F_{1}(a+1, b ; c+1 ; x)=(c-a){ }_{2} F_{1}(a, b ; c+1 ; x)+c(x-1){ }_{2} F_{1}(a+1, b ; c ; x)
$$

Specialising this identity to our case, we find

$$
{ }_{2} F_{1}\left(\frac{3 d-1}{2 d}, \frac{1}{2} ; \frac{3}{2} ;(\cos \alpha)^{2}\right)=-\frac{1}{d-1}\left[{ }_{2} F_{1}\left(\frac{d-1}{2 d}, \frac{1}{2} ; \frac{3}{2} ;(\cos \alpha)^{2}\right)-d(\sin \alpha)^{1 / d-1}\right]
$$

By employing this result, it is straightforward to realise that the expression enclosed by the square brackets in $(\mathrm{C} .21)$ is $\mathfrak{g}_{d}(\alpha) /(d-1)$, being $\mathfrak{g}_{d}(\alpha)$ given by (C.11). This observation and (C.10) allow us to write (C.21) in terms of the width $\ell$ of the strip $A$ as follows

$$
\frac{\mathcal{A}\left[\hat{\gamma}_{\varepsilon}\right]}{L_{\mathrm{AdS}}^{d}}=\frac{L_{\|}^{d-1}}{d-1}\left(\frac{1}{\varepsilon^{d-1}}-\frac{\mathfrak{g}_{d}(\alpha)^{d}}{\ell^{d-1}}+O\left(\varepsilon^{d+1}\right)\right)
$$

The expression (5.8) in the main text corresponds to (C.24) specialised to $d=2$.

The other extremal surface occurring in our analysis is the half hyperplane defined by $x=\ell$. This can be observed by considering the extrinsic curvature of a half hyperplane embedded in $\mathbb{H}_{d+1}$ whose normal vector has non vanishing components only along $z$ and $x$. Denoting by $\theta$ the angle between this normal vector and the positive $x$ semi-axis, one finds $\operatorname{Tr} K \propto \cos \theta$ for the trace of the extrinsic curvature of the half hyperplane. This implies that the vertical hyperplane, which has $\theta=0$, is a local minimum for the area functional. 
By introducing also an infrared cutoff $z_{\mathrm{IR}}$ beside the UV cutoff $\varepsilon$, it is straightforward to show that the portion of surface such that $\varepsilon \leqslant z \leqslant z_{\mathrm{IR}}$ reads

$$
\frac{\mathcal{A}\left[\hat{\gamma}_{\varepsilon}\right]}{L_{\mathrm{AdS}}^{d}}=\frac{L_{\|}^{d-1}}{d-1}\left(\frac{1}{\varepsilon^{d-1}}-\frac{1}{z_{\mathrm{IR}}^{d-1}}\right)
$$

The divergent part of $\mathcal{A}\left[\hat{\gamma}_{\varepsilon}\right]$ is the same one occurring in (C.21), as expected. Let us stress that the finite term in (C.25) vanishes as $z_{\mathrm{IR}} \rightarrow \infty$.

Summarising, for $\alpha \in\left(0, \alpha_{c}\right]$ the minimal surface $\hat{\gamma}_{A}$ is the vertical half hyperplane $x=\ell$ because the surface characterised by (C.14) is not well defined. In the range $\alpha \in$ $\left(\alpha_{c}, \pi / 2\right]$ both the surface given by (C.14) and the vertical half hyperplane $x=\ell$ are well defined extremal solutions of the area functional and, by comparing (C.24) with (C.25), we conclude that $\hat{\gamma}_{A}$ is the one characterised by (C.14). Instead, when $\alpha \in(\pi / 2, \pi)$ the vertical half hyperplane is not a solution anymore of our problem because it does not intersect $\mathcal{Q}$ orthogonally; therefore the minimal surface $\hat{\gamma}_{A}$ is again the surface corresponding to (C.14).

Putting these observations together, we find the following area for the restriction to $z \geqslant \varepsilon$ of the minimal surface corresponding to the strip adjacent to the boundary

$$
\frac{\mathcal{A}\left[\hat{\gamma}_{\varepsilon}\right]}{L_{\mathrm{AdS}}^{d}}=L_{\|}^{d-1}\left[\frac{1}{(d-1) \varepsilon^{d-1}}+\frac{a_{0, d}(\alpha)}{(d-1) \ell^{d-1}}+o(1)\right]
$$

where

$$
a_{0, d}(\alpha) \equiv\left\{\begin{array}{cc}
-\mathfrak{g}_{d}(\alpha)^{d} & \alpha \geqslant \alpha_{c}(d) \\
0 & \alpha \leqslant \alpha_{c}(d)
\end{array}\right.
$$

Notice that $a_{0, d}(\alpha)$ and its first derivative are continuous functions of $\alpha$. Also the higher order derivatives of $a_{0, d}(\alpha)$ are continuous until the $d$-th derivative of $a_{0, d}(\alpha)$, which is discontinuous at $\alpha=\alpha_{c}(d)$. In (5.9) we have specialised (C.26) and (C.27) to $d=2$.

We find it interesting to discuss separately the $d=1$ case. As already remarked below (C.11), in this case we have that $\mathfrak{g}_{1}(\alpha)=1$ identically; therefore a critical value for $\alpha$ does not occur. Moreover, the profile (C.14) simplifies to $(x(\theta), z(\theta))=\ell(\cos \theta, \sin \theta)$. This curve is an arc of circumference of radius $\ell$; therefore it intersects orthogonally the half line $\mathcal{Q}$ given by (4.3) which passes through the origin. We also have that $z_{*}=\ell \sin \alpha$, which corresponds to (C.10) for $d=1$.

As for the length of this arc of circumference with opening angle $\pi-\alpha$ and for $z \geqslant \varepsilon$, it is straightforward to find that

$$
\frac{\mathcal{A}\left[\hat{\gamma}_{\varepsilon}\right]}{L_{\mathrm{AdS}}^{d}}=\int_{\theta_{\varepsilon}}^{\pi-\alpha} \frac{d \theta}{\sin \theta}=\left.\log \left(\frac{\sin (\theta / 2)}{\cos (\theta / 2)}\right)\right|_{\theta_{\varepsilon}} ^{\pi-\alpha}=\log (\ell / \varepsilon)+\log (2 \cot (\alpha / 2))+O\left(\varepsilon^{2}\right)
$$

where the angular cutoff $\theta_{\varepsilon}$ is defined by requiring that $\varepsilon=\ell \sin \theta_{\varepsilon}$. As for the extremal curve given by the half line $x=\ell$, by introducing the IR cutoff $z_{\mathrm{IR}}$, for the length of the part of this straight line such that $\varepsilon \leqslant z \leqslant z_{\mathrm{IR}}$ we find

$$
\frac{\mathcal{A}\left[\hat{\gamma}_{\varepsilon}\right]}{L_{\mathrm{AdS}}^{d}}=\log (\ell / \varepsilon)+\log \left(z_{\mathrm{IR}} / \ell\right)
$$

where the term $\log \left(z_{\mathrm{IR}} / \ell\right)$ diverges when $z_{\mathrm{IR}} / \ell \rightarrow+\infty$. Thus the minimal curve is always given by the arc of circumference. This is consistent with the observation that a critical slope does not occur when $d=1$. 


\section{Infinite strip parallel to the boundary in generic dimension}

In this appendix we consider a strip $A$ parallel to the boundary $x=0$ and at finite distance from it. Let us denote by $\ell_{A}$ the width of the strip and by $d_{A}$ its distance from the boundary (see figure 11). We will focus on spacetimes having $d>1$. For the case $d=1$ we refer the reader to [73].

The main feature of the holographic entanglement entropy corresponding to this simple domain is the fact that in some range of $\alpha$ two qualitatively different hypersurfaces are local extrema of the area functional; therefore the global minimum between them must be found. One of these candidates is the minimal area surface in $\operatorname{AdS}_{d+2}$ corresponding to the infinite strip found in $[5,6]$ (see the blue solid curve in figure 11). Let us denote this hypersurface by $\hat{\gamma}_{A}^{\text {dis }}$, being disconnected from $\mathcal{Q}$. The second candidate $\hat{\gamma}_{A}^{\text {con }}$ is made by the union of two disjoint hypersurfaces like the ones discussed in the appendix $\mathrm{C}$.

When $\alpha \leqslant \alpha_{c}$, we have that $\hat{\gamma}_{A}^{\text {con }}$ is the union of the vertical half hyperplanes defined by $x=d_{A}$ and $x=d_{A}+\ell_{A}$. Instead, for $\alpha>\alpha_{c}$ the hypersurface $\hat{\gamma}_{A}^{\text {con }}$ is made by two disjoint hypersurfaces characterised by the profile (C.14) which depart from the edges of $A$ and intersect $\mathcal{Q}$ orthogonally (see the green solid curves in figure 11 for a case with $\alpha>\alpha_{c}$ ). Furthermore, when $\alpha \leqslant \pi / 2$ the solution $\hat{\gamma}_{A}^{\text {dis }}$ always exists, while one can wonder whether this is the case also for $\alpha>\pi / 2$, where $\mathcal{Q}$ can intersect $\hat{\gamma}_{A}^{\text {dis }}$. This issue is discussed below.

Let us focus first on finding the regime where $\hat{\gamma}_{A}^{\text {con }}$ is the global minimum. The auxiliary domain corresponding to $\hat{\gamma}_{A}^{\text {con }}$ is made by two parallel and disjoint infinite strips $A_{\text {aux }}=$ $A \cup A^{\prime}$ in $\mathbb{R}^{d}$ and the corresponding minimal surface in $\hat{\gamma}_{A \text {,aux }} \subset \mathbb{H}_{d+1}$ has been studied e.g. in [90]. Denoting by $\ell^{\prime}$ the width of $A^{\prime}$ and by $d_{\text {aux }}$ the separation between $A$ and $A^{\prime}$, from figure 11 and (C.16) it is not difficult to realise that

$$
\ell^{\prime}=2 \frac{\sqrt{\pi} \Gamma\left(\frac{d+1}{2 d}\right)}{\Gamma\left(\frac{1}{2 d}\right) \mathfrak{g}_{d}(\alpha)} \ell_{A} \quad d_{\text {aux }}=2 \frac{\sqrt{\pi} \Gamma\left(\frac{d+1}{2 d}\right)}{\Gamma\left(\frac{1}{2 d}\right) \mathfrak{g}_{d}(\alpha)} d_{A}
$$

Taking the part $z \geqslant \varepsilon$ of $\hat{\gamma}_{A}^{\text {dis }}$ and $\hat{\gamma}_{A}^{\text {con }}$, and evaluating the corresponding area as $\varepsilon \rightarrow 0^{+}$, one finds that the area law term is the same; therefore we have to compare the $O(1)$ terms to find $\hat{\gamma}_{A}$. By employing (C.26) and the well known result for the holographic entanglement entropy of the infinite strip in $\operatorname{AdS}_{d+2}[5,6]$, one finds that the expansion of the area of $\hat{\gamma}_{\varepsilon}$ as $\varepsilon \rightarrow 0^{+}$reads

$$
\frac{\mathcal{A}\left[\hat{\gamma}_{\varepsilon}\right]}{L_{\mathrm{AdS}}^{d}}=\frac{L_{\|}^{d-1}}{d-1}\left(\frac{2}{\varepsilon^{d-1}}+\frac{1}{\ell_{A}^{d-1}} \min \left[h_{d}, a_{0, d}(\alpha)\left(\frac{1}{\delta_{A}^{d-1}}+\frac{1}{\left(\delta_{A}+1\right)^{d-1}}\right)\right]+o(1)\right)
$$

The function $a_{0, d}(\alpha)$ has been introduced in (C.27), while the constant $h_{d}$ is defined as $[5,6]$

$$
h_{d} \equiv-2^{d} \pi^{d / 2}\left(\frac{\Gamma\left(\frac{d+1}{2 d}\right)}{\Gamma\left(\frac{1}{2 d}\right)}\right)^{d}
$$

The first term in the argument of the minimisation function occurring in the r.h.s. of (D.2) corresponds to $\hat{\gamma}_{A}^{\text {dis }}$, while the second one comes from $\hat{\gamma}_{A}^{\text {con }}$. Thus, $\hat{\gamma}_{A}=\hat{\gamma}_{A}^{\text {dis }}$ when $\delta_{A} \equiv$ $d_{A} / \ell_{A}$ is large enough, while $\hat{\gamma}_{A}=\hat{\gamma}_{A}^{\text {con }}$ if the strip is close enough to the boundary. We 


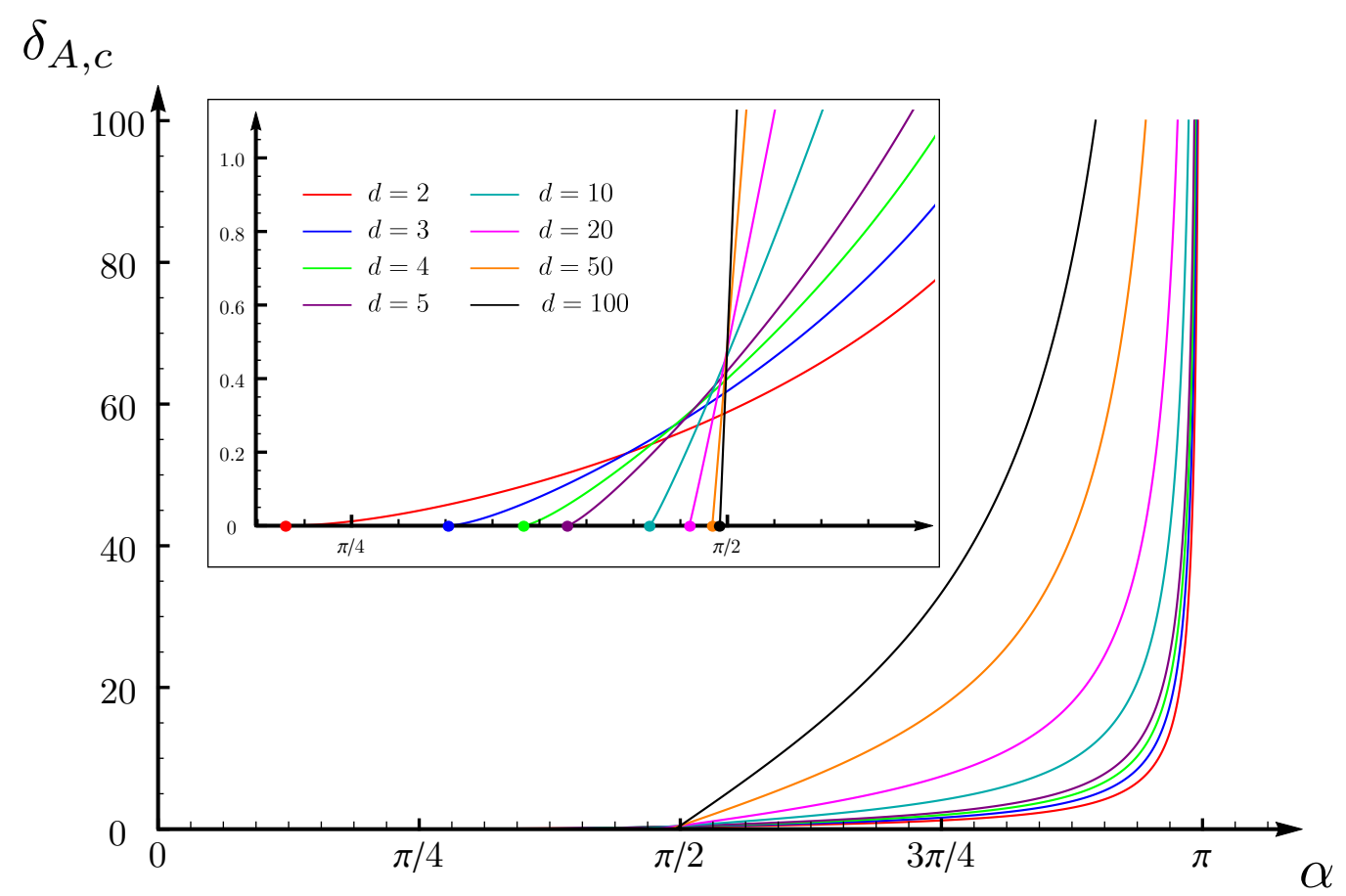

Figure 24. Infinite strip of width $\ell_{A}$ parallel to the boundary at finite distance $d_{A}$ from it: the ratio $\delta_{A}=d_{A} / \ell_{A}$ corresponding to the critical configurations in terms of $\alpha \in\left[\alpha_{c}, \pi\right)$ for some values of $d$. The curves are obtained by finding the unique positive root of (D.4). For $d=2$ and $d=3$ the expression of $\delta_{A, c}$ has been written analytically in (5.11) and (D.7) respectively, while for $d \geqslant 4$ the curves have been found by solving (D.4) numerically.

remark that (D.2) holds for $\alpha \in(0, \pi)$. Notice that, when $\alpha \leqslant \alpha_{c}$, being $h_{d}<0$ and $a_{0, d}(\alpha)=0$, we have that $\hat{\gamma}_{A}=\hat{\gamma}_{A}^{\text {dis }}$.

The critical configurations correspond to the cases where the two terms occurring in the minimisation function of the $O(1)$ term of (D.2) are equal. The value $\delta_{A, c}$ of the ratio $\delta_{A}$ for these configurations can be found as solution of the following equation

$$
\delta_{A, c}^{d-1}\left(\delta_{A, c}+1\right)^{d-1}=\tilde{a}_{0, d}(\alpha)\left[\left(\delta_{A, c}+1\right)^{d-1}+\delta_{A, c}^{d-1}\right] \quad \tilde{a}_{0, d}(\alpha) \equiv \frac{a_{0, d}(\alpha)}{h_{d}}
$$

We remark that $\tilde{a}_{0, d}$ is a positive and non vanishing function of the slope $\alpha$ when $\alpha \in\left(\alpha_{c}, \pi\right)$, while $\tilde{a}_{0, d}(\alpha)=0$ when $\alpha \in\left(0, \alpha_{c}\right]$. This implies that a strictly positive solution of (D.4) does not exist when $\alpha \leqslant \alpha_{c}$, as expected from the fact that $\hat{\gamma}_{A}=\hat{\gamma}_{A}^{\text {dis }}$. Instead, for $\alpha>\alpha_{c}$ we can show that $\delta_{A, c}$ always exists and it is also unique.

The equation (D.4) can be written as $p\left(\delta_{A, c}\right)=0$, where the real polynomial $p\left(\delta_{A, c}\right)$ in powers of $\delta_{A, c}$ schematically reads

$p\left(\delta_{A, c}\right)=\delta_{A, c}^{2(d-1)}+(d-1) \delta_{A, c}^{2 d-3}+\cdots+\left[1-2 \tilde{a}_{0, d}(\alpha)\right] \delta_{A, c}^{d-1}-\tilde{a}_{0, d}(\alpha)(d-1) \delta_{A, c}^{d-2}-\cdots-\tilde{a}_{0, d}(\alpha)$

The maximum number of positive of roots of (D.5) can be determined by employing the Descartes' rule of signs. This rule states that the maximum number of positive roots of a real polynomial is bounded by the number of sign differences between consecutive nonzero 
coefficients of its powers, once they are set in decreasing order (the powers which do not occur must be just omitted). Since $\tilde{a}_{0, d}(\alpha)>0$, the expression (D.5) shows that this number is equal to one in our case; therefore we have at most one positive real root. Its existence is guaranteed by the fact that $p(0)=-\tilde{a}_{0, d}(\alpha)<0$ and $p(\delta) \rightarrow+\infty$ as $\delta \rightarrow+\infty$.

Since $\hat{\gamma}_{A}=\hat{\gamma}_{A}^{\text {dis }}$ when $\alpha \leqslant \alpha_{c}$, as remarked above, the critical configurations exist only for $\alpha \in\left(\alpha_{c}, \pi\right)$. Focussing on this range, an analytic expression for $\delta_{A, c}(\alpha)$ in terms of $\tilde{a}_{0, d}$ for a generic dimension $d$ cannot be found. However, we find it instructive to determine it explicitly for $d=2$ and $d=3$ because (D.4) can be solved in closed form for these cases.

When $d=2$ it is straightforward to obtain the result (5.11) reported in the main text. For $d=3$ the algebraic equation (D.4) has degree four. A shift of the variable allows to write it as follows

$$
u^{4}-\frac{4 \tilde{a}_{0,3}(\alpha)+1}{2} u^{2}+\frac{1-8 \tilde{a}_{0,3}(\alpha)}{16}=0 \quad \delta_{A, c}=u-\frac{1}{2}
$$

which is a biquadratic equation. Its unique positive root reads

$$
\delta_{A, c}=\frac{1}{2}\left(\sqrt{4 \tilde{a}_{0,3}(\alpha)+4 \sqrt{\tilde{a}_{0,3}(\alpha)\left[\tilde{a}_{0,3}(\alpha)+1\right]}+1}-1\right)
$$

For $d \geqslant 4$ the root of (D.4) can be found numerically and the results for some values of $d$ are shown in figure 24, where the curves are defined for $\alpha>\alpha_{c}$ (see the inset, which contains a zoom of the main plot for small values of $\delta_{A, c}$ ).

As briefly anticipated at the beginning of this appendix, when $\alpha>\pi / 2$ one can wonder whether the brane $\mathcal{Q}$ can intersect $\hat{\gamma}_{A}^{\text {dis }}$, leaving $\hat{\gamma}_{A}^{\text {con }}$ as unique solution. Let us denote by $\delta_{A, 0}$ the value of the ratio $d_{A} / \ell_{A}$ characterising the configurations of $A$ such that $\hat{\gamma}_{A}^{\text {dis }}$ is tangent to $\mathcal{Q}$. It is not difficult to realise that $\delta_{A, 0}<\delta_{A, c}$. Indeed, when $\delta_{A}=\delta_{A, 0}$, the surface $\hat{\gamma}_{A}^{\text {dis }}$ can be seen as the union of two surfaces which join smoothly along the intersection with $\mathcal{Q}$. Since these two surfaces connect the boundary at $z=0$ to $\mathcal{Q}$ but they are not orthogonal to $\mathcal{Q}$, the area of $\hat{\gamma}_{A}^{\text {con }}$ is less than the area of $\hat{\gamma}_{A}^{\text {dis }}$ for $\delta_{A}=\delta_{A, 0}$. This argument can be easily applied also for $\delta_{A}<\delta_{A, 0}$; therefore we can conclude that $\hat{\gamma}_{A}^{\text {con }}$ is always the global minimum for $\delta_{A} \leqslant \delta_{A, 0}$.

We find it worth finding also the analytic expression of $\delta_{A, 0}$. The minimal surface $\hat{\gamma}_{A}^{\text {dis }}$ can be obtained by modifying the r.h.s. of (C.14) as follows: first one sets $\alpha=\pi / 2$ and substitutes $\ell$ with $\ell_{A} / 2$, then the resulting $x(\theta)$ is replaced by $x(\theta)+d_{A}+\ell_{A} / 2$. The final result reads

$$
(x(\theta), z(\theta))=\ell_{A}\left(\frac{\cos \theta}{\left(-h_{d}\right)^{1 / d} d}{ }_{2} F_{1}\left(\frac{d-1}{2 d}, \frac{1}{2} ; \frac{3}{2} ;(\cos \theta)^{2}\right)+\delta_{A}+\frac{1}{2}, \frac{(\sin \theta)^{1 / d}}{\left(-h_{d}\right)^{1 / d}}\right)
$$

where $\theta \in[0, \pi]$ and $h_{d}$ is defined in (D.3). In order to impose that $\hat{\gamma}_{A}^{\text {dis }}$ is tangent to $\mathcal{Q}$, we need to find the unit vectors $V^{\mu}$ and $W^{\mu}$ which are tangent to $\hat{\gamma}_{A}^{\text {dis }}$ and $\mathcal{Q}$ respectively, being $\mu \in\{z, x\}$ because of translation invariance along the remaining directions. The vector $V^{\mu}$ can be easily written by setting $\alpha=\pi / 2$ in (C.5) and (C.6), obtaining $V^{\mu}=$ $\frac{1}{z}(-\cos \theta, \sin \theta)$. As for the vector $W^{\mu}$, it reads $W^{\mu}=\frac{1}{z}(\sin \alpha,-\cos \alpha)$. Notice that the intersection is possible only where both $\alpha>\pi / 2$ and $\theta>\pi / 2$. By requiring that $V^{\mu}=W^{\mu}$, 
we find that $\theta_{0}=3 \pi / 2-\alpha$ is the value of the parameter $\theta$ corresponding to the intersection between $\hat{\gamma}_{A}^{\text {dis }}$ and $\mathcal{Q}$. By employing this value, $\delta_{A, 0}$ can be written by imposing that $\hat{\gamma}_{A}^{\text {dis }}$ intersects $\mathcal{Q}$. This condition gives $z\left(\theta_{0}\right)=-x\left(\theta_{0}\right) \tan \alpha$, where $z=z(\theta)$ and $x=x(\theta)$ are given in (D.8). By solving this equation for $\delta_{A}$, we find

$$
\delta_{A, 0}=\frac{1}{\left(-h_{d}\right)^{1 / d}}\left[\frac{\sin \alpha}{d}{ }_{2} F_{1}\left(\frac{d-1}{2 d}, \frac{1}{2} ; \frac{3}{2} ;(\sin \alpha)^{2}\right)-\cot \alpha(-\cos \alpha)^{1 / d}\right]-\frac{1}{2}
$$

A numerical comparison between this analytic expression for $\delta_{A, 0}$ and the curves for $\delta_{A, c}$ leads us to conclude that $\delta_{A, 0}<\delta_{A, c}$ for any value of $d$, as expected from the argument discussed above.

\section{E On a modification for the holographic entanglement entropy}

In section 4 the prescription (4.5) for the holographic entanglement entropy in AdS/BCFT employed throughout this manuscript has been discussed. Considering the minimal area surface anchored to the entangling surface $\partial A \cap \partial B$ in the $z=0$ half hyperplane, since the spacetime has also the boundary $\mathcal{Q}$, it can happen that part of $\partial \hat{\gamma}_{A}$ belongs to $\mathcal{Q}$. Let us denote this hypersurface by $\partial_{\mathcal{Q}} \hat{\gamma}_{A} \equiv \hat{\gamma}_{A} \cap \mathcal{Q}$ and by $\partial_{\mathcal{Q}} \hat{\gamma}_{\varepsilon}$ its restriction to $z \geqslant \varepsilon$. By minimising the area functional in presence of $\mathcal{Q}$ without imposing particular boundary conditions on $\partial_{\mathcal{Q}} \hat{\gamma}_{A}$, one finds that $\hat{\gamma}_{A}$ is the minimal area surface which intersects $\mathcal{Q}$ orthogonally. The prescription (4.5) adopted throughout this manuscript requires to compute the area of such minimal surface $\hat{\gamma}_{A}$ restricted to $z \geqslant \varepsilon$.

In this appendix we consider a possible modification of the prescription for the holographic entanglement entropy suggested in [75]. This proposal includes also the area of $\partial_{\mathcal{Q}} \hat{\gamma}_{\varepsilon}$ as follows

$$
S_{A}=\frac{1}{4 G_{\mathrm{N}}}\left(\mathcal{A}\left[\hat{\gamma}_{\varepsilon}\right]+a_{\mathcal{Q}} L_{\mathrm{AdS}} \mathcal{A}\left[\partial_{\mathcal{Q}} \hat{\gamma}_{\varepsilon}\right]\right)
$$

where $a_{\mathcal{Q}}$ is a dimensionless parameter which might depend on $\mathcal{Q}$ (i.e. on $\alpha$ in our case) but is independent on the region $A$. We remark that in [75] the slope $\alpha$ is fixed to $\alpha=\pi / 2$, but in the following discussion we keep $\alpha$ generic.

Our first observation is that for some domains $A$ the expression (E.1) leads to a discontinuous holographic entanglement entropy as the size of $A$ changes. In particular, this discontinuity occurs whenever two local minima of the area functional compete to determine the global minimum $\hat{\gamma}_{A}$ and only one of them intersects $\mathcal{Q}$. At the critical configuration both of them provide the same value for the area $\mathcal{A}\left[\hat{\gamma}_{\varepsilon}\right]$. Thus, deforming $A$ in a smooth way passing through the critical configuration, we have that on one side of the transition the term $\mathcal{A}\left[\partial_{\mathcal{Q}} \hat{\gamma}_{\varepsilon}\right] \neq 0$ because $\hat{\gamma}_{A}$ intersects $\mathcal{Q}$, while on the other side of the transition $\mathcal{A}\left[\partial_{\mathcal{Q}} \hat{\gamma}_{\varepsilon}\right]=0$ because $\hat{\gamma}_{A} \cap \mathcal{Q}=\emptyset$. Thus, if we require that the holographic entanglement entropy must be a continuous function in terms of the size of the region $A$, then $a_{\mathcal{Q}}$ should vanish.

In the following discussion we employ the strong subadditivity of the entanglement entropy to show that $a_{\mathcal{Q}}=0$. Our argument is based on a choice of domains made by infinite strips which are adjacent to the boundary or parallel to the boundary at a finite distance from it. 
The minimal area surface $\hat{\gamma}_{A}$ for these strips has been described in the appendices $\mathrm{C}$ and D. Here we have to consider also $\mathcal{A}\left[\partial_{\mathcal{Q}} \hat{\gamma}_{\varepsilon}\right]$ for these domains. In particular, from the discussions reported in the appendices $\mathrm{C}$ and $\mathrm{D}$, it is straightforward to realise that the only quantity we miss is $\mathcal{A}\left[\partial_{\mathcal{Q}} \hat{\gamma}_{\varepsilon}\right]$ for a strip adjacent to the boundary when $\alpha>\alpha_{c}$. For these values of $\alpha$ we have that $\partial_{\mathcal{Q}} \hat{\gamma}_{A} \equiv \hat{\gamma}_{A} \cap \mathcal{Q}$ is characterised by the point $P_{*}=\left(x_{*}, z_{*}\right)$ in the two dimensional space described by the coordinates $(x, z)$. In particular, notice that $\partial_{\mathcal{Q}} \hat{\gamma}_{A} \equiv \hat{\gamma}_{A} \cap \mathcal{Q}$ does not reach $z=0$ because we are dealing with infinite strips.

The metric induced on $\partial_{\mathcal{Q}} \hat{\gamma}_{\varepsilon}$ from (4.2) is given by

$$
d s^{2}=\frac{L_{\mathrm{AdS}}^{2}}{z_{*}^{2}}\left(d y_{1}^{2}+\cdots+d y_{d-1}^{2}\right)
$$

This leads to

$$
\mathcal{A}\left[\partial_{\mathcal{Q}} \hat{\gamma}_{\epsilon}\right]=\frac{1}{z_{*}^{d-1}} L_{\mathrm{AdS}}^{d-1} L_{\|}^{d-1}=L_{\mathrm{AdS}}^{d-1} L_{\|}^{d-1} \frac{a_{d, *}}{(d-1) \ell^{d-1}} \quad a_{d, *}(\alpha) \equiv \frac{(d-1) \mathfrak{g}_{d}(\alpha)^{d-1}}{(\sin \alpha)^{1-1 / d}}
$$

Let us assume that $\alpha>\alpha_{c}$. Furthermore, we need (E.1) for a strip adjacent and parallel to the boundary.

Given an infinite strip $A$ of width $\ell$ which is adjacent to the boundary, from (C.26) and (E.3) we find that (E.1) becomes

$$
\frac{\mathcal{A}\left[\hat{\gamma}_{\varepsilon}\right]+a_{\mathcal{Q}} L_{\mathrm{AdS}} \mathcal{A}\left[\partial_{\mathcal{Q}} \hat{\gamma}_{\varepsilon}\right]}{L_{\mathrm{AdS}}^{d}}=\frac{L_{\|}^{d-1}}{d-1}\left(\frac{1}{\varepsilon^{d-1}}+\frac{a_{\mathcal{Q}} a_{d, *}(\alpha)-\mathfrak{g}_{d}(\alpha)^{d}}{\ell^{d-1}}+o(1)\right)
$$

As for an infinite strip $A$ of width $\ell_{A}$ parallel to the boundary at a distance $d_{A}$ from it (i.e. whose points have $d_{A} \leqslant x \leqslant d_{A}+\ell_{A}$, as shown in figure 11), from (D.2) and (E.4) we obtain

$$
\begin{aligned}
& \frac{\mathcal{A}\left[\hat{\gamma}_{\varepsilon}\right]+a_{\mathcal{Q}} L_{\mathrm{AdS}} \mathcal{A}\left[\partial_{\mathcal{Q}} \hat{\gamma}_{\varepsilon}\right]}{L_{\mathrm{AdS}}^{d}}= \\
& \quad=\frac{L_{\|}^{d-1}}{d-1}\left(\frac{2}{\varepsilon^{d-1}}+\min \left[\frac{h_{d}}{\ell_{A}^{d-1}},\left(a_{\mathcal{Q}} a_{d, *}(\alpha)-\mathfrak{g}_{d}(\alpha)^{d}\right)\left(\frac{1}{d_{A}^{d-1}}+\frac{1}{\left(d_{A}+\ell_{A}\right)^{d-1}}\right)\right]+o(1)\right)
\end{aligned}
$$

Our argument is based on the strong subadditivity of the entanglement entropy, which states that, given spatial domains $A_{1}$ and $A_{2}$ such that $A_{1} \cap A_{2} \neq \emptyset$, the following inequality must hold [33]

$$
S_{A_{1}}+S_{A_{2}} \geqslant S_{A_{1} \cup A_{2}}+S_{A_{1} \cap A_{2}}
$$

By employing this inequality for special configurations where $A_{1}$ and $A_{2}$ are two infinite strips and by assuming that the holographic entanglement entropy is given by (E.1), in the following we show that $a_{\mathcal{Q}}=0$. Let us denote by $\ell_{j}$ the width of $A_{j}$ and by $d_{j}$ its distance from the boundary (namely the points of $A_{j}$ have $d_{j} \leqslant x \leqslant d_{j}+\ell_{j}$ ), being $j \in\{1,2\}$. We can assume $d_{1}<d_{2}$ without loss of generality. Notice that the area law terms of the four terms involved in the inequality (E.6) always simplify.

In the first configuration of infinite strips that we consider, $d_{1}=0$ and the remaining parameters are such that the corresponding configuration of minimal surfaces in the bulk 

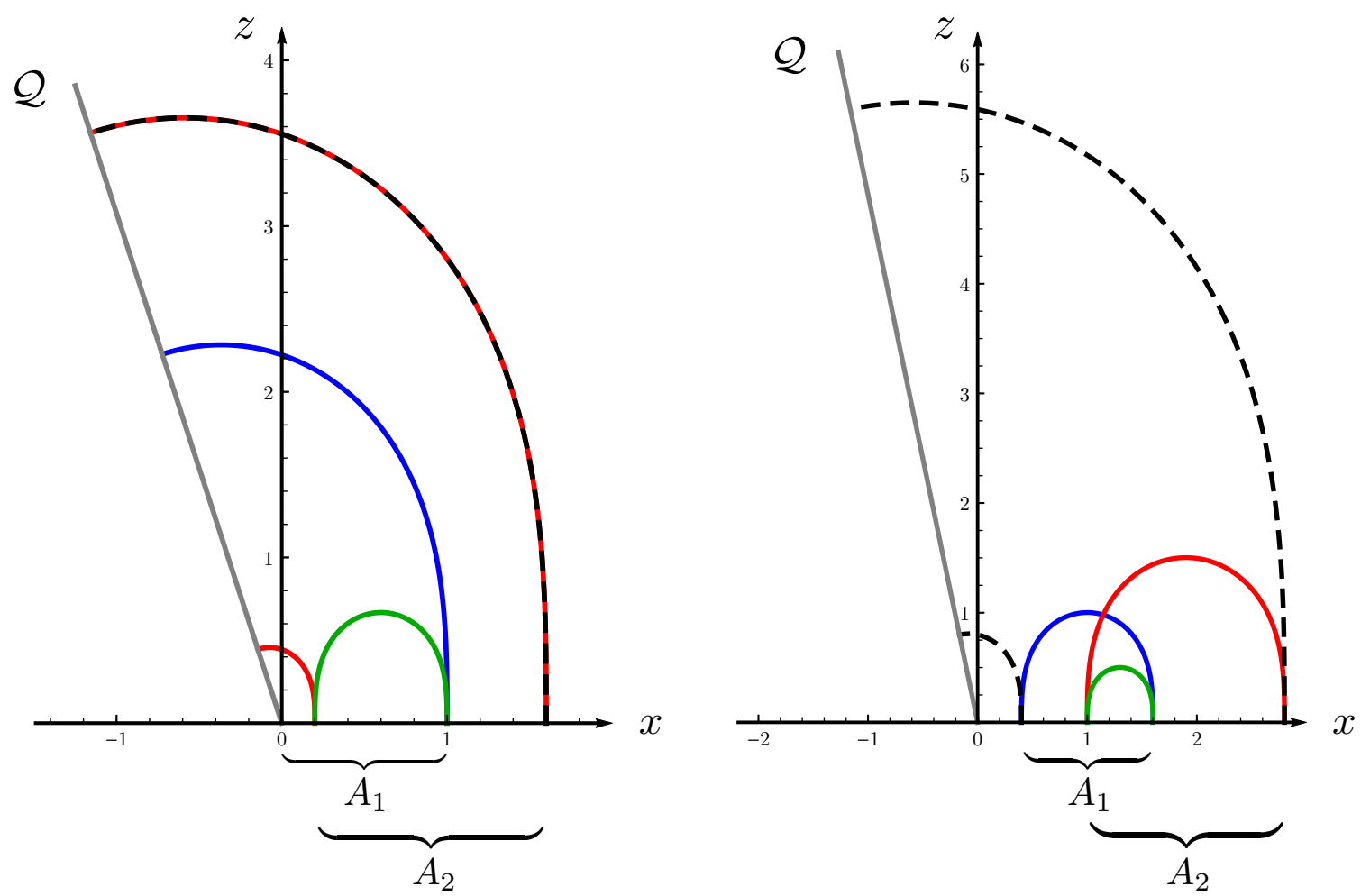

Figure 25. Configurations of two infinite strips $A_{1}$ and $A_{2}$ such that $A_{1} \cap A_{2} \neq \emptyset$, with the corresponding minimal surfaces, which have been employed in section E. The blue solid curve corresponds to $\hat{\gamma}_{A_{1}}$, the red solid curve (which is made by two components in the left panel) to $\hat{\gamma}_{A_{2}}$, the green solid curve to $\hat{\gamma}_{A_{1} \cap A_{2}}$ and the black dashed curve (which is made by one component in the left panel and by two components in the right panel) to $\hat{\gamma}_{A_{1} \cup A_{2}}$. Left: $A_{1}$ is adjacent to the boundary while $A_{2}$ is parallel to the boundary at a finite distance from it. Right: both $A_{1}$ and $A_{2}$ are parallel to the boundary at finite distances from it.

is like the one depicted in the left panel of figure 25. This means that $d_{2} / \ell_{2} \leqslant \delta_{A, c}$ and $d_{2} /\left(\ell_{1}-d_{2}\right) \geqslant \delta_{A, c}$, being $\delta_{A, c}$ the unique positive root of (D.4) (see figure 24). Moreover, we also have the geometrical constraints given by $d_{2}<\ell_{1}<d_{2}+\ell_{2}$. By assuming that the prescription (E.1) holds, let us consider the inequality (E.6) for the configuration depicted in the left panel of figure 25. By employing (E.4) and (E.5), we find

$$
\frac{a_{\mathcal{Q}} a_{d, *}(\alpha)-\mathfrak{g}_{d}(\alpha)^{d}}{\ell_{1}^{d-1}}+\frac{a_{\mathcal{Q}} a_{d, *}(\alpha)-\mathfrak{g}_{d}(\alpha)^{d}}{d_{2}^{d-1}} \geqslant \frac{h_{d}}{\left(\ell_{1}-d_{2}\right)^{d-1}}
$$

where we remark that the term coming from $S_{A_{1} \cup A_{2}}$ simplifies with the term originated from the component of $\hat{\gamma}_{A_{2}}$ anchored to $x=d_{2}+\ell_{2}$. This cancellation occurs between the terms corresponding to the two curves which overlap in figure 25 . Isolating $a_{\mathcal{Q}}$ on one side of the inequality (E.7), one finds

$$
\frac{h_{d}}{\left(\ell_{1}-d_{2}\right)^{d-1}}+\mathfrak{g}_{d}(\alpha)^{d}\left(\frac{1}{\ell_{1}^{d-1}}+\frac{1}{d_{2}^{d-1}}\right) \leqslant a_{\mathcal{Q}} a_{d, *}(\alpha)\left(\frac{1}{\ell_{1}^{d-1}}+\frac{1}{d_{2}^{d-1}}\right)
$$

By using (C.24), we recognise in the 1.h.s. of this inequality the combination of terms which provides the critical configuration for the strip $A_{1} \cap A_{2}$ parallel to the boundary. 
Furthermore, since $d_{2} /\left(\ell_{1}-d_{2}\right) \geqslant \delta_{A, c}$ for the configuration we are considering, the l.h.s. of (E.8) is negative. In particular, by choosing the parameters such that $d_{2} /\left(\ell_{1}-d_{2}\right) \rightarrow \delta_{A, c}^{+}$, we find that the l.h.s. of (E.8) vanishes in the limit. Being $a_{d, *}(\alpha)>0$, we have that $a_{\mathcal{Q}} \geqslant 0$.

A similar analysis can be performed by considering the configuration depicted in the right panel of figure 25, where $A_{1}$ and $A_{2}$ are two infinite strips parallel to the boundary and $d_{1}>0$. The geometrical constraint for this configuration is $d_{2}<d_{1}+\ell_{1}<d_{2}+\ell_{2}$. Instead, in order to find the configuration of minimal surfaces shown in the right panel of figure 25 , we must require that $d_{1} / \ell_{1} \geqslant \delta_{A, c}, d_{2} / \ell_{2} \geqslant \delta_{A, c}, d_{1} /\left(d_{2}+\ell_{2}-d_{1}\right) \leqslant \delta_{A, c}$ and also $d_{2} /\left(d_{1}+\ell_{1}-d_{2}\right) \geqslant \delta_{A, c}$. By employing (E.4) and (E.5), the strong subadditivity inequality (E.6) for the configuration of infinite strips in the right panel of figure 25 provides the following inequality

$$
\frac{h_{d}}{\ell_{1}^{d-1}}+\frac{h_{d}}{\ell_{2}^{d-1}} \geqslant \frac{h_{d}}{\left(d_{1}+\ell_{1}-d_{2}\right)^{d-1}}+\left(a_{\mathcal{Q}} a_{d, *}(\alpha)-\mathfrak{g}_{d}(\alpha)^{d}\right)\left(\frac{1}{d_{1}^{d-1}}+\frac{1}{\left(d_{2}+\ell_{2}\right)^{d-1}}\right)
$$

which can be conveniently rearranged as

$$
\begin{aligned}
h_{d}\left(\frac{1}{\ell_{1}^{d-1}}+\frac{1}{\ell_{2}^{d-1}}-\frac{1}{\left(d_{1}+\ell_{1}-d_{2}\right)^{d-1}}\right)+\mathfrak{g}_{d}(\alpha)^{d} & \left(\frac{1}{d_{1}^{d-1}}+\frac{1}{\left(d_{2}+\ell_{2}\right)^{d-1}}\right) \\
& \geqslant a_{\mathcal{Q}} a_{d, *}(\alpha)\left(\frac{1}{d_{1}^{d-1}}+\frac{1}{\left(d_{2}+\ell_{2}\right)^{d-1}}\right)
\end{aligned}
$$

Considering the special case of $\ell_{1}=\ell_{2}$ first and then taking the limit $d_{2} \rightarrow d_{1}^{+}$, we have that $A_{1}$ and $A_{2}$ tends to overlap. In this limit, the r.h.s. of (E.10) becomes the combination occurring in the holographic entanglement entropy of an infinite strip parallel to the boundary (see (D.2) and (C.27)). In particular, given the configuration in the right panel of figure 25, the l.h.s. of (E.10) in this limit is a positive quantity which is proportional to the combination that appears in (D.4). Since in this limit the constraints introduced above (E.9) saturate, the l.h.s. of (E.10) becomes arbitrarily closed to $0^{+}$. Combining this observation with $a_{d, *}(\alpha)>0$, we obtain $a_{\mathcal{Q}} \leqslant 0$.

Thus, by employing the strong subadditivity inequality (E.6) for the configurations depicted in figure 25 , we can conclude that $a_{\mathcal{Q}}=0$ in (E.1).

\section{F On the infinite wedge adjacent to the boundary}

In this appendix we provide the technical details underlying the computation of the holographic entanglement entropy of the infinite wedge $A$ adjacent to the boundary. The main results have been collected and discussed in section 6 .

In the half plane $\left\{(x, y) \in \mathbb{R}^{2}, x \geqslant 0\right\}$, let us introduce the polar coordinates $(\rho, \phi)$ such that $\phi=0$ is the half line given by $x=0$ and $y>0$, namely

$$
x=\rho \sin \phi \quad y=\rho \cos \phi
$$

In terms of these coordinates, the infinite wedge $A$ having one of its two edges on the boundary $x=0$ can be described without loss of generality as follows

$$
A=\{(\rho, \phi) \mid 0 \leqslant \phi \leqslant \gamma, \rho \leqslant L\} \quad L \gg \varepsilon
$$



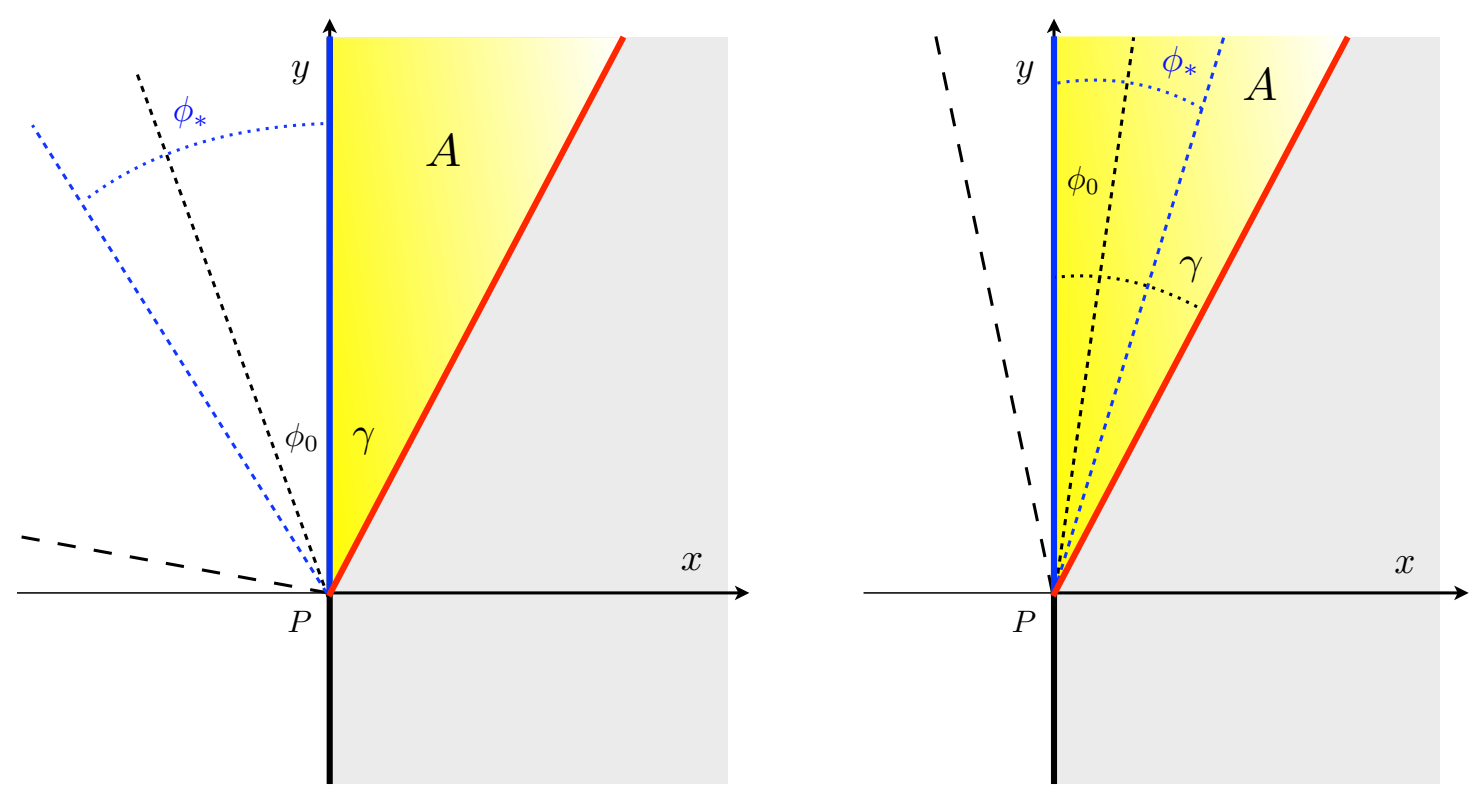

Figure 26. The opening angles occurring in the construction of the minimal surface $\hat{\gamma}_{A}$ anchored to the infinite wedge $A$ adjacent to the boundary with opening angle $\gamma$, which has been discussed in section 6 and in the appendix F. In the left panel $\alpha \in(0, \pi / 2]$ and in the right panel $\alpha \in[\pi / 2, \pi)$. The wedge $A$ is the yellow region, whose edges are the red and the blue solid half lines, given by $\phi=\gamma$ and $\phi=0$ respectively. The auxiliary wedge $A_{\text {aux }}$ is the infinite wedge in $\mathbb{R}^{2}$ containing $A$ whose tip is $P$ and whose edges are the red half line and the black dashed line with the largest dashing. The black dashed line with the smallest dashing at $\phi=\phi_{0}$ corresponds to the bisector of $A_{\text {aux }}$. The blue dashed half line at $\phi=\phi_{*}$ corresponds to the projection of $\hat{\gamma}_{A} \cap \mathcal{Q}$ in the $z=0$ plane.

In order to study the holographic entanglement entropy (4.5) of the infinite wedge $A$ within the $\mathrm{AdS}_{4} / \mathrm{BCFT}_{3}$ setup described in section 4, let us consider the surfaces anchored to the edge $\{(\rho, \phi) \mid \phi=\gamma\}$ of $A$ and embedded in the region of $\mathbb{H}_{3}$ defined by (4.4). The symmetry under dilatations tells us that $\hat{\gamma}_{A}$ belongs to the class of surfaces $\gamma_{A}$ described by (6.1) with $q(\phi)>0$. The metric induced on $\gamma_{A}$ from $\mathbb{H}_{3}$, whose metric is (3.1), reads

$$
\left.d s^{2}\right|_{\gamma_{A}}=L_{\mathrm{AdS}}^{2}\left(\frac{1+q^{2}}{\rho^{2}} d \rho^{2}-\frac{2 q^{\prime}}{\rho q} d \rho d \phi+\frac{\left(q^{\prime}\right)^{2}+q^{4}}{q^{2}} d \phi^{2}\right)
$$

Our analysis heavily relies on [27], where the authors have found the minimal area surface in $\mathbb{H}_{3}$ anchored to both the edges of an infinite wedge. Indeed, we study $\hat{\gamma}_{A}$ by introducing an auxiliary wedge $A_{\text {aux }}$ in the $z=0$ boundary of $\mathbb{H}_{3}$ such that $A \subsetneq A_{\text {aux }}$ and $\{(\rho, \phi) \mid \phi=\gamma\}$ is a common edge of both $A$ and $A_{\text {aux }}$. Considering the minimal area surface $\hat{\gamma}_{A \text {,aux }}$ in $\mathbb{H}_{3}$ anchored to the edges of $A_{\text {aux }}$, the minimal area surface $\hat{\gamma}_{A}$ anchored to the edge $\{(\rho, \phi) \mid \phi=\gamma\}$ of $A$ and intersecting $\mathcal{Q}$ orthogonally is the part of $\hat{\gamma}_{A \text {,aux }}$ identified by the constraint (4.4). Thus, finding $\hat{\gamma}_{A}$ corresponds to find the proper $\hat{\gamma}_{A \text {,aux }}$.

In figure 26 we show the relevant angles occurring in our construction, by distinguishing the two cases of $\alpha \in(0, \pi / 2]$ (left panel) and $\alpha \in[\pi / 2, \pi)$ (right panel). The infinite wedge $A$ adjacent to the boundary $x=0$ is the yellow region, which is embedded into the grey half plane $x \geqslant 0$. The edges of the auxiliary wedge $A_{\text {aux }}$ are the red half line $\{(\rho, \phi) \mid \phi=\gamma\}$ 
and the half line denoted by the black large dashing. The bisector of $A_{\text {aux }}$ is the black dashed half line at $\phi=\phi_{0}$; therefore the opening angle of $A_{\text {aux }}$ is $2\left(\gamma-\phi_{0}\right)$. The half line corresponding to the black small dashing is the bisector of the auxiliary wedge, while the blue dashed half line is the projection on the $z=0$ plane of the half line given by $\hat{\gamma}_{A} \cap \mathcal{Q}$.

\section{F.1 Minimal surface condition}

The metric (F.3) induced on the surfaces $\gamma_{A}$ leads to the following area functional

$$
\frac{\mathcal{A}\left[\gamma_{A}\right]}{L_{\mathrm{AdS}}^{2}}=\int_{\gamma_{A}} \frac{1}{\rho} \sqrt{q^{\prime 2}+q^{2}+q^{4}} d \phi d \rho=\int_{\gamma_{A}} \frac{1}{\rho} \mathcal{L} d \phi d \rho \quad \mathcal{L} \equiv \sqrt{q^{\prime 2}+q^{2}+q^{4}}
$$

The functions $q(\phi)$ characterising the extrema of this functional can be found by observing that its integrand is independent of $\phi$. The first integral associated to this invariance provides a quantity which is independent of $\phi$. It reads

$$
\frac{\partial \mathcal{L}}{\partial q^{\prime}} q^{\prime}-\mathcal{L} \propto \frac{q^{4}+q^{2}}{\sqrt{\left(q^{\prime}\right)^{2}+q^{4}+q^{2}}}
$$

Let us introduce the angle $\phi_{0}$ such that

$$
q^{\prime}\left(\phi_{0}\right)=0 \quad q\left(\phi_{0}\right) \equiv q_{0} \quad q_{0}>0
$$

The angle $\phi_{0}$ provides the bisector of the auxiliary wedge $A_{\text {aux }}$.

By employing (F.6) into the condition that (F.5) is independent of $\phi$, one obtains the following first order differential equation

$$
\frac{q^{4}+q^{2}}{\sqrt{\left(q^{\prime}\right)^{2}+q^{4}+q^{2}}}=\sqrt{q_{0}^{4}+q_{0}^{2}}
$$

Taking the square of this expression, one gets

$$
\frac{\left(q^{\prime}\right)^{2}}{q^{2}}=\left(q^{2}+1\right)\left(\frac{q^{4}+q^{2}}{q_{0}^{4}+q_{0}^{2}}-1\right) \quad q \geqslant q_{0}
$$

Separating the variables in (F.8), one finds $d \phi=\mathcal{P}_{\phi}\left(q, q_{0}\right) d q$. Then, by integrating the latter expression, we get

$$
\left|\phi-\phi_{0}\right|=\int_{q_{0}}^{q} \mathcal{P}_{\phi}\left(\hat{q}, q_{0}\right) d \hat{q}=P\left(q, q_{0}\right) \quad \mathcal{P}_{\phi}\left(q, q_{0}\right) \equiv \frac{\sqrt{q_{0}^{4}+q_{0}^{2}}}{q \sqrt{\left(q^{2}+1\right)\left(q^{2}-q_{0}^{2}\right)\left(q^{2}+q_{0}^{2}+1\right)}}
$$

where $q \geqslant q_{0}$ and $P\left(q, q_{0}\right)$ has been written in (6.6). From (F.9), it is straightforward to realise that $P\left(q_{0}, q_{0}\right)=0$ and that the function $P\left(q, q_{0}\right) \geqslant 0$ is an increasing function of $q \geqslant q_{0}$. The minimal area surface $\hat{\gamma}_{A}$ is described by (6.1) with the proper $q(\phi)$ obtained by inverting (F.9).

The opening angle of the auxiliary wedge $A_{\text {aux }}$ is $2\left(\gamma-\phi_{0}\right)$, as already observed above from figure 26. This angle can be found from (F.9) as follows

$$
\gamma-\phi_{0}=\int_{\phi_{0}}^{\gamma} d \phi=\int_{q_{0}}^{\infty} \mathcal{P}_{\phi}\left(\tilde{q}, q_{0}\right) d \tilde{q}=\lim _{q \rightarrow+\infty} P\left(q, q_{0}\right) \equiv P_{0}\left(q_{0}\right)
$$

Equivalent expressions of $P_{0}\left(q_{0}\right)$ have been reported in (3.4) and (6.8).

The next step of our analysis consists in studying the intersection $\hat{\gamma}_{A} \cap \mathcal{Q}$ and the opening angle of $A_{\text {aux }}$. 


\section{F.2 Intersection between the minimal surface and the brane}

In order to find the extremal surface $\hat{\gamma}_{A}$ anchored to the edge $\{(\rho, \phi) \mid \phi=\gamma\}$ of $A$ and ending on the half plane $\mathcal{Q}$, beside the differential equation (F.7) we also have to impose that $\hat{\gamma}_{A}$ and $\mathcal{Q}$ intersect orthogonally.

By writing the equation (4.3) for $\mathcal{Q}$ in terms of the polar coordinates (F.1) and intersecting the resulting expression with the ansatz (6.1) for $\gamma_{A}$, we find

$$
q_{*} \sin \phi_{*}=-\cot \alpha \quad q_{*} \equiv q\left(\phi_{*}\right)
$$

This relation defines the angle $\phi=\phi_{*}$ at which $\gamma_{A}$ and $\mathcal{Q}$ intersect. Thus, $\gamma_{A} \cap \mathcal{Q}$ is the half line whose points have coordinates $(z, \rho, \phi)=\left(\rho / q_{*}, \rho, \phi_{*}\right)$, with $\rho>0$. Since $q_{*}>0$, from (F.11) we have that $\phi_{*} \leqslant 0$ when $\alpha \in(0, \pi / 2]$, and $\phi_{*} \geqslant 0$ when $\alpha \in[\pi / 2, \pi)$. This is shown in figure 26, where the blue dashed half line corresponds to the projection of $\hat{\gamma}_{A} \cap \mathcal{Q}$ on the $z=0$ plane. The relation (F.11) tells us that $\phi_{*}=0$ when $\alpha=\pi / 2$, as expected.

In order to impose that $\gamma_{A}$ and $\mathcal{Q}$ intersect orthogonally along the half line at $\phi=\phi_{*}$, we have to find the unit vector normal to $\gamma_{A}$ and the unit vector normal to the $\mathcal{Q}$. The surfaces $\gamma_{A}$ described by the ansatz (6.1) can be equivalently written as $\mathcal{C}=0$, with $\mathcal{C} \equiv z-\rho / q(\phi)$. Thus, the unit vector normal to $\gamma_{A}$ is

$$
n_{\mu}=\frac{\partial_{\mu} \mathcal{C}}{\sqrt{g^{\alpha \beta} \partial_{\alpha} \mathcal{C} \partial_{\beta} \mathcal{C}}}=\frac{L_{\mathrm{AdS}}}{z \sqrt{\left(q^{\prime}\right)^{2}+q^{4}+q^{2}}}\left(q^{2},-q, \rho q^{\prime}\right)
$$

where the components of the vector have been ordered according to $\mu \in\{z, \rho, \phi\}$. As for the half plane $\mathcal{Q}$, its definition in (4.3) can be written as $\mathcal{C}_{\mathcal{Q}}=0$, with $\mathcal{C}_{\mathcal{Q}} \equiv z+\rho \sin \phi \tan \alpha$, where the first relation in (F.1) has been used. This tells us that the unit vector normal to the half plane $\mathcal{Q}$ is

$$
b_{\mu}=\frac{\partial_{\mu} \mathcal{C}_{\mathcal{Q}}}{\sqrt{g^{\alpha \beta} \partial_{\alpha} \mathcal{C}_{\mathcal{Q}} \partial_{\beta} \mathcal{C}_{\mathcal{Q}}}}=\frac{L_{\mathrm{AdS}} \cos \alpha}{z}(1, \sin \phi \tan \alpha, \rho \cos \phi \tan \alpha)
$$

Given the unit vectors (F.12) and (F.13), we have to impose that they are orthogonal (namely $g^{\mu \nu} n_{\mu} b_{\nu}=0$ ) along the half line $\gamma_{A} \cap \mathcal{Q}$ at $\phi=\phi_{*}$. This requirement leads to the following relation

$$
q_{*}^{2}+\left[q_{*}^{\prime} \cos \phi_{*}-q_{*} \sin \phi_{*}\right] \tan \alpha=0 \quad q_{*}^{\prime} \equiv q^{\prime}\left(\phi_{*}\right)
$$

which can be written also as

$$
\frac{q_{*}^{\prime}}{q_{*}}=\tan \phi_{*}-\frac{q_{*}}{\cos \phi_{*}} \cot \alpha
$$

Taking the square of (F.15) first and then employing (F.8) to write $\left(q_{*}^{\prime} / q_{*}\right)^{2}$ in terms of $q_{*}$ and $q_{0}$, we have

$$
\left(\tan \phi_{*}-\frac{q_{*}}{\cos \phi_{*}} \cot \alpha\right)^{2}=\left(q_{*}^{2}+1\right)\left(\frac{q_{*}^{4}+q_{*}^{2}}{q_{0}^{4}+q_{0}^{2}}-1\right)
$$


This expression can be simplified by using (F.11) to rewrite $q_{*}$ in terms of $\phi_{*}$, finding

$$
\left(\tan \phi_{*}\right)^{2}\left[\frac{(\cot \alpha)^{2}}{\left(\sin \phi_{*}\right)^{2}}+1\right]=\frac{1}{q_{0}^{4}+q_{0}^{2}} \frac{(\cot \alpha)^{2}}{\left(\sin \phi_{*}\right)^{2}}\left[\frac{(\cot \alpha)^{2}}{\left(\sin \phi_{*}\right)^{2}}+1\right]-1
$$

This relation leads to the following biquadratic equation

$$
q_{0}^{4}+q_{0}^{2}=\left[1+\frac{(\cot \alpha)^{2}}{\left(\sin \phi_{*}\right)^{2}}\right](\cos \alpha)^{2}\left(\cot \phi_{*}\right)^{2}
$$

which has only one positive root in terms of $q_{0}^{2}$. This solution allows us to write $q_{0}$ in terms of $\phi_{*}$ as follows

$$
q_{0}=\frac{1}{\sqrt{2}}\left(\sqrt{1+4\left[1+(\cot \alpha)^{2}\left(\csc \phi_{*}\right)^{2}\right](\cos \alpha)^{2}\left(\cot \phi_{*}\right)^{2}}-1\right)^{1 / 2}
$$

Instead of $\phi_{*}$, we prefer to adopt $q_{0}$ as fundamental parameter; therefore let us consider the biquadratic equation in terms of $\sin \phi_{*}$ obtained from (F.18), namely

$$
\left[1+\frac{q_{0}^{4}+q_{0}^{2}}{(\cos \alpha)^{2}}\right]\left(\sin \phi_{*}\right)^{4}-\left[1-(\cot \alpha)^{2}\right]\left(\sin \phi_{*}\right)^{2}-(\cot \alpha)^{2}=0
$$

whose positive solution for $\left(\sin \phi_{*}\right)^{2} \equiv s_{*}\left(\alpha, q_{0}\right)^{2}$ reads

$$
\begin{aligned}
& s_{*}\left(\alpha, q_{0}\right)^{2}= \\
& \quad=\frac{1}{2}\left(1+\frac{q_{0}^{4}+q_{0}^{2}}{(\cos \alpha)^{2}}\right)^{-1}\left[1-(\cot \alpha)^{2}+\sqrt{\left[1-(\cot \alpha)^{2}\right]^{2}+4\left(1+\frac{q_{0}^{4}+q_{0}^{2}}{(\cos \alpha)^{2}}\right)(\cot \alpha)^{2}}\right]
\end{aligned}
$$

Notice that $s_{*}\left(\pi-\alpha, q_{0}\right)^{2}=s_{*}\left(\alpha, q_{0}\right)^{2}$. We denote by $s_{*}\left(\alpha, q_{0}\right)>0$ the positive root of (F.21), which has been written explicitly in (6.2). Plugging $s_{*}\left(\alpha, q_{0}\right)$ into (F.11), one obtains (6.4).

Since $\phi_{*} \leqslant \phi_{0} \leqslant 0$ when $\alpha \in(0, \pi / 2]$, while $0 \leqslant \phi_{0} \leqslant \phi_{*}$ when $\alpha \in[\pi / 2, \pi)$ (see figure 26), we find it convenient to introduce $\eta_{\alpha} \equiv-\operatorname{sign}(\cot \alpha)$, as done in (6.2). Then, the expression for $\phi_{*}=\phi_{*}\left(q_{0}, \alpha\right)$ in (6.3) can be written straightforwardly. Furthermore, (F.9) leads to

$$
\left|\phi_{*}-\phi_{0}\right|=\int_{q_{0}}^{q_{*}} \mathcal{P}_{\phi}\left(q, q_{0}\right) d q=P\left(q_{*}, q_{0}\right)= \begin{cases}\phi_{0}-\phi_{*} & 0<\alpha \leqslant \pi / 2 \\ \phi_{*}-\phi_{0} & \pi / 2 \leqslant \alpha<\pi\end{cases}
$$

This provides the angle $\phi_{0}=\phi_{0}\left(q_{0}, \alpha\right)$ as follows

$$
\phi_{0}=\phi_{*}\left(q_{0}, \alpha\right)-\eta_{\alpha} P\left(q_{*}\left(\alpha, q_{0}\right), q_{0}\right)=\eta_{\alpha}\left(\arcsin \left[s_{*}\left(\alpha, q_{0}\right)\right]-P\left(q_{*}, q_{0}\right)\right)
$$

where the last step has been obtained by using $\phi_{*}\left(q_{0}, \alpha\right)$ in (6.3). Notice that $\phi_{0}$ characterises the opening angle of the auxiliary wedge $A_{\text {aux }}$.

Finally, an expression for the opening angle $\gamma$ in terms of $\alpha$ and $q_{0}$ can be written. Indeed, from (F.10) one first finds that $\gamma=P_{0}\left(q_{0}\right)+\phi_{0}$; then (F.23) can be used to get (6.5).

Summarising, we have determined the angles $\phi_{*}, \phi_{0}$ and $\gamma$ as functions of $\alpha$ and $q_{0}$. They are given in (6.3), (F.23) and (6.5) respectively. 


\section{F.3 Area of the minimal surface}

The minimal surface $\hat{\gamma}_{A}$ anchored to the edge $\{(\rho, \phi) \mid \phi=\gamma\}$ of the infinite wedge adjacent to the boundary given by (F.2) is non compact; therefore we have to compute the area of its restriction $\hat{\gamma}_{\varepsilon}$ to $z \geqslant \varepsilon$. We stress that $\hat{\gamma}_{A} \subsetneq \hat{\gamma}_{A \text {,aux }}$ is the part of the auxiliary minimal surface $\hat{\gamma}_{A \text {,aux }}$ identified by the constraint (4.4), as discussed in section 6 and in the beginning of the appendix $\mathrm{F}$ (see also figure 26). The auxiliary infinite wedge $A_{\text {aux }}$ and the corresponding minimal surface $\hat{\gamma}_{A \text {,aux }}$ have been obtained through the analysis of the appendices F.1 and F.2. The area of $\hat{\gamma}_{\varepsilon \text {,aux }} \equiv \hat{\gamma}_{A \text {,aux }} \cap\{z \geqslant \varepsilon\}$ has been computed in [27].

We compute $\mathcal{A}\left[\hat{\gamma}_{\varepsilon}\right]$ by considering two parts of $\hat{\gamma}_{A \text {,aux }}$, that we denote by $\hat{\gamma}_{A \text {,aux }}^{\infty}$ and $\hat{\gamma}_{A, \text { aux }}^{*}$.

The surface $\hat{\gamma}_{A \text {,aux }}^{\infty}$ corresponds to the part of $\hat{\gamma}_{A \text {,aux }}$ such that with $\phi_{0} \leqslant \phi \leqslant \gamma$. We remark that $\hat{\gamma}_{A \text {,aux }}^{\infty}$ reaches the half plane at $z=0$ along the edge at $\phi=\gamma$ and it corresponds to half of $\hat{\gamma}_{A \text {,aux }}$. The surface $\hat{\gamma}_{A \text {,aux }}^{*}$ is the part of $\hat{\gamma}_{A \text {,aux }}$ having $\phi_{*} \leqslant \phi \leqslant \phi_{0}$ when $\alpha \in(0, \pi / 2]$ and $\phi_{0} \leqslant \phi \leqslant \phi_{*}$ when $\alpha \in[\pi / 2, \pi)$ (see respectively the left and right panel of figure 26). Notice that $\hat{\gamma}_{A \text {,aux }}^{*}=\emptyset$ when $\alpha=\pi / 2$.

The restrictions of $\hat{\gamma}_{A \text {,aux }}^{\infty}$ and $\hat{\gamma}_{A \text {,aux }}^{*}$ to $z \geqslant \varepsilon$ provide $\hat{\gamma}_{\varepsilon \text {,aux }}^{\infty}$ and $\hat{\gamma}_{\varepsilon, \text { aux }}^{*}$ respectively, and we denote their areas by $L_{\text {AdS }}^{2} \mathcal{A}_{\infty}$ and $L_{\text {AdS }}^{2} \mathcal{A}_{*}$ respectively. From (F.4), one finds

$$
\mathcal{A}_{\infty} \equiv \int_{\hat{\gamma}_{\varepsilon}^{\infty}} \frac{1}{\rho} \sqrt{q^{\prime 2}+q^{2}+q^{4}} d \phi d \rho \quad \mathcal{A}_{*} \equiv \int_{\hat{\gamma}_{\varepsilon}^{*}} \frac{1}{\rho} \sqrt{q^{\prime 2}+q^{2}+q^{4}} d \phi d \rho
$$

which give the area of $\hat{\gamma}_{\varepsilon}$ as follows

$$
\frac{\mathcal{A}\left[\hat{\gamma}_{\varepsilon}\right]}{L_{\mathrm{AdS}}^{2}}= \begin{cases}\mathcal{A}_{\infty}+\mathcal{A}_{*} & 0<\alpha \leqslant \pi / 2 \\ \mathcal{A}_{\infty}-\mathcal{A}_{*} & \pi / 2 \leqslant \alpha<\pi\end{cases}
$$

By using (F.7) and (F.9), the angular part of the integrands in (F.24) can be written as

$$
\sqrt{q^{\prime 2}+q^{2}+q^{4}} \frac{d q}{\left|q^{\prime}\right|}=\frac{q^{4}+q^{2}}{\sqrt{q_{0}^{4}+q_{0}^{2}}} \mathcal{P}_{\phi}\left(q, q_{0}\right) d q=\frac{\sqrt{q^{4}+q^{2}}}{\sqrt{q^{4}+q^{2}-q_{0}^{4}-q_{0}^{2}}} d q
$$

which leads us to introduce the following function

$$
\int_{q_{0}}^{q} \frac{\sqrt{\hat{q}^{4}+\hat{q}^{2}}}{\sqrt{\hat{q}^{4}+\hat{q}^{2}-q_{0}^{4}-q_{0}^{2}}} d \hat{q} \equiv \mathcal{G}\left(q, q_{0}\right) \quad q \geqslant q_{0}
$$

Performing explicitly this integral, we obtain

$$
\mathcal{G}\left(q, q_{0}\right) \equiv-\mathrm{i} \sqrt{q_{0}^{2}+1} \mathbb{E}\left(\mathrm{i} \operatorname{arcsinh} \sqrt{\frac{q^{2}-q_{0}^{2}}{1+2 q_{0}^{2}}} \mid \frac{2 q_{0}^{2}+1}{q_{0}^{2}+1}\right)
$$

which satisfies the condition $\mathcal{G}\left(q_{0}, q_{0}\right)=0$, as expected from (F.27). By employing the following identity [100]

$$
\begin{aligned}
\mathbb{E}(\mathrm{i} \psi \mid m)= & \mathrm{i} \mathbb{F}(\arctan (\sinh \psi) \mid 1-m)-\mathrm{i} \mathbb{E}(\arctan (\sinh \psi) \mid 1-m) \\
& +\mathrm{i} \sqrt{1-(1-m) \tanh ^{2} \psi} \sinh \psi
\end{aligned}
$$


we can write (F.28) in a form which does not contain the imaginary unit, finding the real expression reported in (6.11).

Since $\hat{\gamma}_{A \text {,aux }}^{\infty}$ is half of $\hat{\gamma}_{A \text {,aux }}$, the area $\mathcal{A}_{\infty}$ has been already computed in [27]. First we have to expand (F.28) for large $q$, finding

$$
\mathcal{G}\left(q, q_{0}\right)=q-F\left(q_{0}\right)+O\left(1 / q^{3}\right) \quad q \gg 1 \quad q \gg q_{0}
$$

where $F\left(q_{0}\right)$ has been explicitly written in (3.3). In order to get the area $\mathcal{A}_{\infty}$, a large cutoff $\rho_{\max } \gg 1$ in the radial direction must be introduced. Then, we have

$$
\varepsilon=\frac{\rho_{\min }}{q_{0}}, \quad \varepsilon=\frac{\rho_{\max }}{q\left(\gamma-\delta_{\varepsilon}\right)}, \quad L=\rho_{\max } \cos \delta_{\varepsilon},
$$

where $\delta_{\varepsilon} \sim 0^{+}$is the angle between the edge of $A$ at $\phi=\gamma$ and the straight line in the $z=0$ half plane connecting the tip of the wedge to the intersection point between the circumference given by $\rho=\rho_{\max }$ and the projection of $\partial \hat{\gamma}_{\varepsilon} \cap\{z=\varepsilon\}$ on the $z=0$ half plane. By employing the expansion (F.30) and (F.31), the area $\mathcal{A}_{\infty}$ is obtained as follows [27]

$$
\begin{aligned}
\mathcal{A}_{\infty} & =\int_{\rho_{\min }}^{\rho_{\max }} \frac{d \rho}{\rho} \int_{q_{0}}^{\rho / \varepsilon} \frac{\sqrt{q^{4}+q^{2}}}{\sqrt{q^{4}+q^{2}-q_{0}^{4}-q_{0}^{2}}} d q=\int_{\rho_{\min }}^{\rho_{\max }} \frac{\mathcal{G}\left(\rho / \varepsilon, q_{0}\right)}{\rho} d \rho \\
& =\int_{\rho_{\min }}^{\rho_{\max }} \frac{1}{\rho}\left[\frac{\rho}{\varepsilon}-F\left(q_{0}\right)+O\left((\varepsilon / \rho)^{3}\right)\right] d \rho=\frac{\rho_{\max }-\rho_{\min }}{\varepsilon}-F\left(q_{0}\right) \log \left(\rho_{\max } / \rho_{\min }\right)+\ldots \\
& =\frac{L}{\varepsilon}-F\left(q_{0}\right) \log (L / \varepsilon)+\ldots
\end{aligned}
$$

where the dots correspond to finite terms for $\varepsilon \rightarrow 0^{+}$. We remark that $\mathcal{A}_{\infty}$ provides the expected linear divergence (area law term) whose coefficient is the length of the entangling curve $\partial A \cap \partial B$. Furthermore, the coefficient of the subleading logarithmic divergence is half of the corresponding coefficient (3.2) found for the wedge in $\mathrm{AdS}_{4} / \mathrm{CFT}_{3}$, as expected, being $\hat{\gamma}_{A \text {,aux }}^{\infty}$ half of $\hat{\gamma}_{A \text {,aux }}$.

The computation of the surface integral $\mathcal{A}_{*}$ in (F.24) is similar to the one of $\mathcal{A}_{\infty}$, with a crucial difference in the angular integral. In particular, we find

$$
\begin{aligned}
\mathcal{A}_{*} & =\int_{\rho_{\min }}^{\rho_{\max }} \frac{d \rho}{\rho} \int_{q_{0}}^{q_{*}} \frac{\sqrt{q^{4}+q^{2}}}{\sqrt{q^{4}+q^{2}-q_{0}^{4}-q_{0}^{2}}} d q=\int_{\rho_{\min }}^{\rho_{\max }} \frac{\mathcal{G}\left(q_{*}, q_{0}\right)}{\rho} d \rho \\
& =\mathcal{G}\left(q_{*}, q_{0}\right) \log \left(\rho_{\max } / \rho_{\min }\right)=\mathcal{G}\left(q_{*}, q_{0}\right) \log (L / \varepsilon)+\ldots
\end{aligned}
$$

Notice that the double integral in $\mathcal{A}_{*}$ factorises into the product of two integrals that can be computed separately. This simplification does not occur in the computation of $\mathcal{A}_{\infty}$.

Finally, plugging (F.32) and (F.33) into (F.25), we find the total corner function $F_{\alpha}$ in terms of $\alpha$ and $q_{0}$, whose explicit expression has been reported in (6.10). Combining this formula with (6.5), we obtain $F_{\alpha}(\gamma)$ parametrically through the real parameter $q_{0}>0$. This function is the main result of this manuscript. It is shown in figure 14 and figure 15 .

A considerable simplification occurs in the expressions obtained above when $\alpha=\pi / 2$. Indeed, being $q_{*}>0$, the relation (F.11) tells us that $\phi_{*}=0$. Then, since $0 \leqslant\left|\phi_{0}\right| \leqslant\left|\phi_{*}\right|$, 
we have that $\phi_{*}=\phi_{0}=0$, and this implies $q_{*}=q_{0}$. By substituting $\phi_{0}=0$ into (F.10), we can conclude that $\gamma=P_{0}\left(q_{0}\right)$ in this special case. As for the corner function, the condition $q_{*}=q_{0}$ tells us that $\mathcal{G}\left(q_{*}, q_{0}\right)=\mathcal{G}\left(q_{0}, q_{0}\right)=0$. Plugging this result in (6.10), we find that $F_{\pi / 2}=F\left(q_{0}\right)$. Thus, when $\alpha=\pi / 2$ we have that the minimal surface $\hat{\gamma}_{A}$ is half of the minimal surface found in [27], namely $\hat{\gamma}_{A}=\hat{\gamma}_{A \text {,aux }}^{\infty}$ as expected. This is also stated in (6.12).

\section{F.4 On the limiting regimes of the corner function}

We find it worth considering some interesting regimes of the corner function $F_{\alpha}(\gamma)$, whose analytic expression is given by (6.5) and (6.10). In particular, we focus on the limits $\gamma \rightarrow 0$ and $\gamma \rightarrow \pi / 2$, which correspond to $q_{0} \rightarrow+\infty$ and $q_{0} \rightarrow 0$ respectively. The main results derived in the following are discussed also in section 6.1.

In order to expand $\gamma\left(q_{0}\right)$ in (6.5) for small and large values of $q_{0}$, we find it convenient to write it as follows

$$
\gamma=P_{0}\left(q_{0}\right)+\int_{\pi / 2}^{\alpha} \partial_{\tilde{\alpha}} \gamma d \tilde{\alpha}
$$

where (6.12) has been used and $P_{0}\left(q_{0}\right)$ is given by (3.4) or (6.8). From (6.5) we have that the integrand in (F.34) reads

$$
\partial_{\alpha} \gamma=\eta_{\alpha}\left(\partial_{\alpha} \arcsin \left[s_{*}\left(\alpha, q_{0}\right)\right]-\partial_{\alpha} P\left(q_{*}\left(\alpha, q_{0}\right), q_{0}\right)\right)
$$

where $s_{*}\left(\alpha, q_{0}\right)$ in the first term is given by (6.2). Then, (F.9) tells us that $P\left(q_{*}\left(\alpha, q_{0}\right), q_{0}\right)$ depends on $\alpha$ only through its first argument $q_{*}\left(\alpha, q_{0}\right)$, which is also the upper extremum in the integral defining $P\left(q, q_{0}\right)$.

Thus, for the second term in (F.35) with $\alpha \in(0, \pi)$ we find

$$
\begin{gathered}
\partial_{\alpha} P\left(q_{*}\left(\alpha, q_{0}\right), q_{0}\right)=\left.\partial_{\alpha}\left(q_{*}\left(\alpha, q_{0}\right)\right) \mathcal{P}_{\phi}\left(q, q_{0}\right)\right|_{q=q_{*}\left(\alpha, q_{0}\right)} \\
=\frac{\sqrt{q_{0}^{4}+q_{0}^{2}}}{\sqrt{\left(q_{*}^{2}\left(\alpha, q_{0}\right)+1\right)\left(q_{*}^{2}\left(\alpha, q_{0}\right)-q_{0}^{2}\right)\left(q_{*}^{2}\left(\alpha, q_{0}\right)+q_{0}^{2}+1\right)}} \frac{\partial_{\alpha}\left(q_{*}\left(\alpha, q_{0}\right)\right)}{q_{*}\left(\alpha, q_{0}\right)} \\
=-\eta_{\alpha} \frac{\sqrt{q_{*}^{2}\left(\alpha, q_{0}\right)-(\cot \alpha)^{2}}}{\left(q_{*}^{2}\left(\alpha, q_{0}\right)+1\right) q_{*}\left(\alpha, q_{0}\right)} \partial_{\alpha}\left(q_{*}\left(\alpha, q_{0}\right)\right) \tan \alpha
\end{gathered}
$$

We remark that the combination $(\tan \alpha) \partial_{\alpha} q_{*}\left(\alpha, q_{0}\right)$ in the last expression is regular when $\alpha \rightarrow \pi / 2$. Similarly, for the first term in (F.35) we find

$$
\partial_{\alpha} \arcsin \left[s_{*}\left(\alpha, q_{0}\right)\right]=\partial_{\alpha} \arcsin \left[\frac{|\cot \alpha|}{q_{*}\left(\alpha, q_{0}\right)}\right]=\eta_{\alpha} \frac{\cot \alpha \partial_{\alpha} q_{*}\left(\alpha, q_{0}\right)+(\csc \alpha)^{2} q_{*}\left(\alpha, q_{0}\right)}{q_{*}\left(\alpha, q_{0}\right) \sqrt{q_{*}^{2}\left(\alpha, q_{0}\right)-(\cot \alpha)^{2}}}
$$

It is important to observe that the factor $\eta_{\alpha}$ in (F.36) and (F.37) simplify with the analogous one in (F.35). Thus, it becomes evident that (F.35) is smooth for $\alpha \in(0, \pi)$.

To study $\gamma$ for small and large values of $q_{0}$, we first employ (F.35) and (F.36) for the integrand in (F.34); then we expand the resulting expression in the regime we are interested in and only at the end we integrate the coefficients of the expansion. 

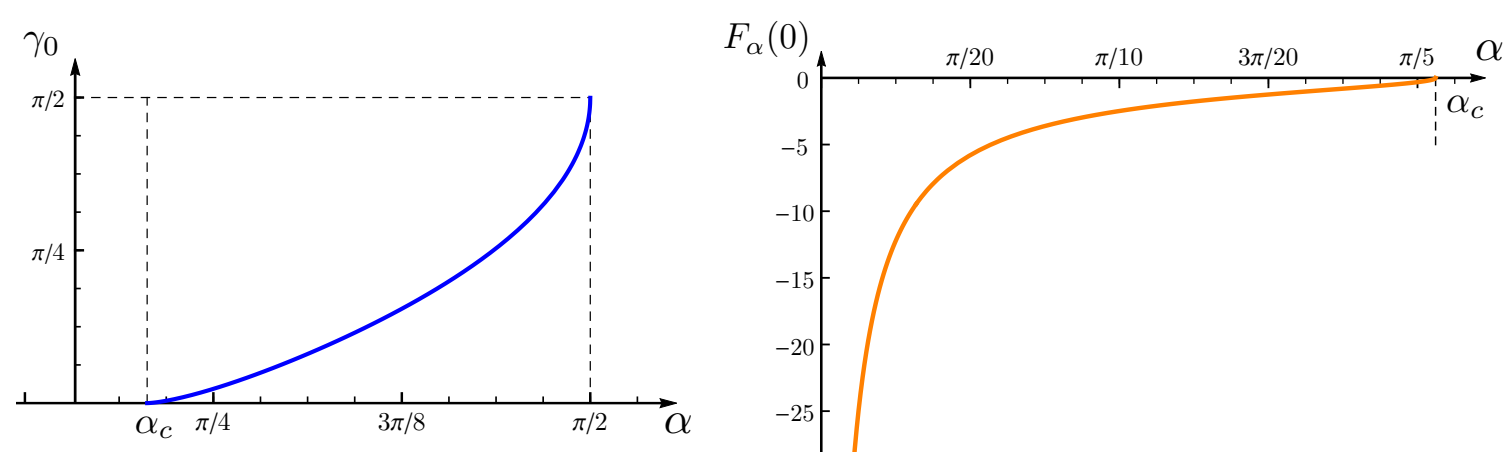

Figure 27. Left: the function $\gamma_{0}(\alpha)$ for $\alpha \in\left[\alpha_{c}, \pi / 2\right]$, being $\gamma_{0}$ defined by $F_{\alpha}\left(\gamma_{0}\right)=0$. Right: the function $F_{\alpha}(0)$ in terms of $\alpha \leqslant \alpha_{c}$.

The corner function $F_{\alpha}\left(q_{0}\right)$ in (6.10) can be treated in the same way. First, by employing (6.12), we write $F_{\alpha}$ as

$$
F_{\alpha}=F\left(q_{0}\right)-\int_{\alpha}^{\pi / 2} \partial_{\tilde{\alpha}} F_{\tilde{\alpha}} d \tilde{\alpha}
$$

Then, from the derivative of (6.10) with respect to $\alpha$, the integral representation of $\mathcal{G}\left(q, q_{0}\right)$ in (F.27) and the expression of $q_{*}\left(\alpha, q_{0}\right)$ in (6.4), we find that

$$
\begin{aligned}
\partial_{\alpha} F_{\alpha} & =\left.\eta_{\alpha}\left(\partial_{\alpha} q_{*}\left(\alpha, q_{0}\right)\right) \partial_{q} \mathcal{G}\left(q, q_{0}\right)\right|_{q=q_{*}\left(\alpha, q_{0}\right)} \\
& =\eta_{\alpha} \frac{\sqrt{q_{*}^{4}\left(\alpha, q_{0}\right)+q_{*}^{2}\left(\alpha, q_{0}\right)}}{\sqrt{q_{*}^{4}\left(\alpha, q_{0}\right)+q_{*}^{2}\left(\alpha, q_{0}\right)-q_{0}^{4}-q_{0}^{2}}} \partial_{\alpha}\left(q_{*}\left(\alpha, q_{0}\right)\right) \\
& =\eta_{\alpha} \frac{|\sec \alpha| q_{*}\left(\alpha, q_{0}\right)}{\sqrt{1+q_{*}^{2}\left(\alpha, q_{0}\right)}} \partial_{\alpha}\left(q_{*}\left(\alpha, q_{0}\right)\right)=-\frac{q_{*}\left(\alpha, q_{0}\right)}{\cos \alpha \sqrt{1+q_{*}^{2}\left(\alpha, q_{0}\right)}} \partial_{\alpha}\left(q_{*}\left(\alpha, q_{0}\right)\right)
\end{aligned}
$$

where, like in (F.36), we observe again the occurrence of $\left(\partial_{\alpha} q_{*}\left(\alpha, q_{0}\right)\right) / \cos \alpha$, which is finite and regular when $\alpha=\pi / 2$. Thus, also in this case $\eta_{\alpha}$ simplifies; therefore it becomes evident that $\partial_{\alpha} F_{\alpha}$ is a smooth function in $\alpha \in(0, \pi)$.

By plugging (F.39) into (F.38), we obtain an expression which can be easily expanded for $q_{0} \rightarrow 0$ and $q_{0} \rightarrow+\infty$. Only at the end one integrates the coefficients of the expansion as prescribed in the r.h.s. of (F.38). We remark that the analysis presented here holds for any $\alpha \in(0, \pi)$.

Before considering the regimes $\gamma \rightarrow 0$ and $\gamma \rightarrow \pi / 2$ of the corner function, we find it worth remarking that when $\alpha \in\left[\alpha_{c}, \pi / 2\right]$ the corner function $F_{\alpha}(\gamma)$ has a unique zero (see figure 14), as already discussed in section 6. Denoting by $\gamma_{0}$ the value of $\gamma$ such that $F_{\alpha}\left(\gamma_{0}\right)=0$, the function $\gamma_{0}(\alpha)$ in terms of $\alpha \in\left[\alpha_{c}, \pi / 2\right]$ can be obtained numerically and the result is shown in the left panel of figure 27 . 


\section{F.4.1 Large $q_{0}$ regime}

Let us consider the limit $q_{0} \rightarrow+\infty$ of the opening angle $\gamma\left(q_{0}\right)$ written in the form (F.34). For the first term, which is given by (3.4) or (6.8), we find

$$
P_{0}\left(q_{0}\right)=\frac{1}{\sqrt{2 \pi} \Gamma\left(\frac{3}{4}\right)^{2}}\left(\frac{\Gamma\left(\frac{3}{4}\right)^{4}}{q_{0}}+\frac{\pi^{2}-6 \Gamma\left(\frac{3}{4}\right)^{4}}{24 q_{0}^{3}}+\frac{16 \Gamma\left(\frac{3}{4}\right)^{4}-5 \pi^{2}}{160 q_{0}^{5}}+O\left(1 / q_{0}^{7}\right)\right)
$$

As for the second term in (F.34), the integrand can be expanded by employing (F.35), obtaining

$$
\partial_{\alpha} \gamma=\frac{(\csc \alpha)^{3 / 2}}{2 q_{0}}-\frac{(1+\csc \alpha)(\csc \alpha)^{3 / 2}}{8 q_{0}^{3}}+O\left(1 / q_{0}^{5}\right)
$$

Finally, by plugging (F.40) and (F.41) into (F.34), and integrating separately the coefficients of the resulting expansion, one finds the first expression in (6.14).

The limit $q_{0} \rightarrow+\infty$ of the corner function $F_{\alpha}\left(q_{0}\right)$ can be studied in a similar way, starting from (F.38). As for the first term, whose explicit expression has been reported in (3.3), its expansion reads

$$
F\left(q_{0}\right)=\frac{1}{\sqrt{2 \pi} \Gamma\left(\frac{3}{4}\right)^{2}}\left(\Gamma\left(\frac{3}{4}\right)^{4} q_{0}-\frac{\pi^{2}-2 \Gamma\left(\frac{3}{4}\right)^{4}}{8 q_{0}}+\frac{\pi^{2}}{32 q_{0}^{3}}+O\left(1 / q_{0}^{5}\right)\right)
$$

The second term in (F.38) can be addressed by using (F.39), whose expansion is

$$
\partial_{\alpha} F_{\alpha}=\frac{(\csc \alpha)^{3 / 2}}{2} q_{0}+\frac{(3 \csc \alpha+1)(\csc \alpha)^{3 / 2}}{8 q_{0}}+\frac{(3 \cos (2 \alpha)-12 \sin \alpha+7)(\csc \alpha)^{7 / 2}}{128 q_{0}^{3}}+O\left(1 / q_{0}^{5}\right)
$$

The coefficient of the leading term in this expansion coincides with the coefficient of the leading term in the expansion (F.41), while the subleading terms are different. By inserting the expansions (F.42) and (F.43) into (F.38) first and then integrating the coefficient of the leading term of the resulting expression, one obtains the second expression in (6.14).

As discussed in detail in section 6.1, a peculiar feature of the corner function $F_{\alpha}(\gamma)$ as $\gamma \rightarrow 0^{+}$is that $F_{\alpha}(\gamma) \rightarrow+\infty$ when $\alpha>\alpha_{c}$, while it tends to a finite value $F_{\alpha}(\gamma) \rightarrow F_{\alpha}(0)$ when $\alpha \leqslant \alpha_{c}$. The function $F_{\alpha}(0)$ in terms of $\alpha \leqslant \alpha_{c}$ can be obtained numerically and the result is shown in the right panel of figure 27. In particular, (6.16) holds for the critical slope $\alpha_{c}$, and this feature has been employed to get (7.4) for an infinite wedge which has only its tip on the boundary.

We find it worth discussing also the behaviour of the angle $\phi_{*}$ characterising the half line $\hat{\gamma}_{A} \cap \mathcal{Q}$ as $\gamma \rightarrow 0^{+}$. When $\alpha>\alpha_{c}$, from the expansion of (6.3) as $q_{0} \rightarrow+\infty$ and (6.14) we find that

$$
\phi_{*}=-\frac{\cos \alpha \sqrt{\csc \alpha}}{\mathfrak{g}(\alpha)} \gamma+\ldots \quad \alpha>\alpha_{c}
$$

which implies that $\phi_{*} \rightarrow 0$ when $\gamma \rightarrow 0^{+}$. Instead, when $\alpha \leqslant \alpha_{c}$, we have to consider the value $\hat{q}_{0}$ introduced in section 6.1 and plug it into (6.3). The result is a negative and increasing function of $\alpha$ which takes the value $-\pi / 2$ for $\alpha \rightarrow 0^{+}$and vanishes for $\alpha=\alpha_{c}$. 


\section{F.4.2 Small $q_{0}$ regime}

The method described in the appendix F.4.1 can be adapted to study the limit $q_{0} \rightarrow 0^{+}$of the functions $\gamma\left(q_{0}\right)$ and $F_{\alpha}\left(q_{0}\right)$, once they are written in the form given by (F.34) and (F.38) respectively.

Considering the opening angle $\gamma$, for the first term of (F.34) we find

$$
P_{0}\left(q_{0}\right)=\frac{\pi}{2}-\frac{\pi}{2} q_{0}+\frac{3 \pi}{8} q_{0}^{3}-\frac{61 \pi}{128} q_{0}^{5}+O\left(q_{0}^{7}\right)
$$

As for the expansion of the integrand in (F.34), we find

$$
\partial_{\alpha} \gamma=q_{0}+\frac{5 \cos (2 \alpha)-3}{4} q_{0}^{3}+\frac{63 \cos (4 \alpha)-132 \cos (2 \alpha)+61}{64} q_{0}^{5}+O\left(q_{0}^{7}\right)
$$

Plugging (F.45) and (F.46) into (F.34) first and then integrating the coefficients of the resulting expansion, we find that

$$
\gamma=\frac{\pi}{2}-(\pi-\alpha) q_{0}+\frac{3(\pi-\alpha)+5 \sin \alpha \cos \alpha}{4} q_{0}^{3}+O\left(q_{0}^{5}\right)
$$

which can be inverted obtaining

$$
q_{0}=\frac{\pi / 2-\gamma}{\pi-\alpha}-\frac{6(\pi-\alpha)+5 \sin (2 \alpha)}{8(\pi-\alpha)^{4}}(\pi / 2-\gamma)^{3}+O\left((\pi / 2-\gamma)^{5}\right) \quad \gamma \rightarrow \frac{\pi}{2}
$$

The limit $q_{0} \rightarrow 0^{+}$of the corner function $F_{\alpha}\left(q_{0}\right)$ in the form (F.38) can be studied in the same way. The first term in the r.h.s. of (F.38) is (3.3) and its expansion reads

$$
F\left(q_{0}\right)=\frac{\pi}{4} q_{0}^{2}-\frac{7 \pi}{32} q_{0}^{4}+O\left(q_{0}^{6}\right)
$$

As for the integrand occurring in (F.38), from (F.39) we obtain

$$
\partial_{\alpha} F_{\alpha}=\frac{1}{(\sin \alpha)^{2}}-\frac{1}{2} q_{0}^{2}+\frac{7-15 \cos (2 \alpha)}{16} q_{0}^{4}+O\left(q_{0}^{6}\right)
$$

By inserting (F.49) and (F.50) into (F.38) first and then integrating separately the coefficients of the resulting expansion, we find

$$
F_{\alpha}=-\cot \alpha+\frac{\pi-\alpha}{2} q_{0}^{2}-\frac{7(\pi-\alpha)+15 \cos \alpha \sin \alpha}{16} q_{0}^{4}+O\left(q_{0}^{6}\right)
$$

Finally, by employing (F.48) into (F.51), we obtain

$$
F_{\alpha}(\gamma)=-\cot \alpha+\frac{(\pi / 2-\gamma)^{2}}{2(\pi-\alpha)}+\frac{5(\pi-\alpha+\cos \alpha \sin \alpha)}{16(\pi-\alpha)^{4}}(\pi / 2-\gamma)^{4}+O\left((\pi / 2-\gamma)^{6}\right)
$$

which is one of our main results. In (6.17) the first two terms of (F.52) have been reported. 


\section{F.5 A relation between the infinite wedge and the infinite strip}

In the expansion (6.15) of the holographic corner function $F_{\alpha}(\gamma)$ for $\gamma \rightarrow 0$ and in the $O(1)$ term of the holographic entanglement entropy of the infinite strip adjacent to the boundary in (5.9), the same function $\mathfrak{g}(\alpha)$ given by (5.4) occurs. In the following we explain this connection by exploiting a conformal map which relates the infinite wedge adjacent to the boundary in the half plane and the infinite strip adjacent to a border of a half cylinder. This analysis has been done by adapting to our case in a straightforward way the analogue relation in absence of the boundary, which involves the infinite wedge in $\mathbb{R}^{2}$ and the infinite strip on the surface of an infinite cylinder [41, 59, 93, 94].

Consider a $\mathrm{BCFT}_{3}$ defined on $\mathbb{R}_{+}^{3} \equiv\left\{\left(t_{\mathrm{E}}, x, y\right) \in \mathbb{R}^{3} \mid x \geqslant 0\right\}$ endowed with the usual Euclidean metric $d s^{2}=d t_{\mathrm{E}}^{2}+d x^{2}+d y^{2}$. By adopting the polar coordinates introduced in (F.1), where we recall that $0 \leqslant \phi \leqslant \pi$, this metric becomes $d s^{2}=d t_{\mathrm{E}}^{2}+d \rho^{2}+\rho^{2} d \phi^{2}$. We define $t_{\mathrm{E}}=0$ the slice containing the infinite wedge $A$ adjacent to the boundary introduced in section 6 , whose edges are given by $\phi=0$ and $\phi=\gamma$. By introducing the coordinates $(\tilde{r}, \chi)$ through the relations $t_{\mathrm{E}}=\tilde{r} \cos \chi$ and $\rho=\tilde{r} \sin \chi$, where $\tilde{r} \geqslant 0$ and $0 \leqslant \chi \leqslant \pi$, the flat metric becomes

$$
d s^{2}=d \tilde{r}^{2}+\tilde{r}^{2}\left(d \chi^{2}+(\sin \chi)^{2} d \phi^{2}\right)
$$

Let us define the coordinate $\tau \in \mathbb{R}$ as $\tilde{r}=L_{0} e^{\tau / L_{0}}$. The tip of the wedge $A$ corresponds to $\tau \rightarrow-\infty$, being $\rho=0=\tilde{r}$ in the previous coordinates. In terms of the coordinates $(\tau, \chi, \phi)$, the metric (F.53) reads

$$
d s^{2}=e^{2 \tau / L_{0}} d \tilde{s}^{2} \quad d \tilde{s}^{2} \equiv d \tau^{2}+L_{0}^{2}\left(d \chi^{2}+(\sin \chi)^{2} d \phi^{2}\right)
$$

i.e. the flat metric on $\mathbb{R}_{+}^{3}$ is conformally equivalent to $d \tilde{s}^{2}$, which is the metric $\mathbb{R} \times S_{+}^{2}$, being $S_{+}^{2}$ a two dimensional hemisphere whose radius is $L_{0}$. The condition $t_{\mathrm{E}}=0$ corresponds to $\chi=\pi / 2$ and the metric induced on this slice from $d \tilde{s}^{2}$ is given by $\left.d \tilde{s}^{2}\right|_{\chi=\pi / 2}=d \tau^{2}+L_{0}^{2} d \phi^{2}$, which characterises the external surface of a half cylinder of radius $L_{0}$, whose boundaries are defined by $\phi=0$ and $\phi=\pi$ (see figure 28). Thus, on this surface, the infinite wedge $A$ corresponds to the infinite strip adjacent to the boundary and enclosed by the generatrices given by $\phi=0$ and $\phi=\gamma$ (the yellow region in figure 28). The width of this infinite strip measured along the surface of the cylinder is $\ell=L_{0} \gamma$.

In terms of the coordinates $(\rho, \phi)$ in $\left.\mathbb{R}_{+}^{3}\right|_{t_{\mathrm{E}}=0}$, the entanglement entropy of the infinite wedge $A$ adjacent to the boundary can be written as

$$
S_{A}=b \frac{\rho_{\max }-\rho_{\min }}{\varepsilon}-f_{\alpha}(\gamma) \log \left(\rho_{\max } / \rho_{\min }\right)+O(1)
$$

where $\rho_{\max }=L$ and $\rho_{\min }=\varepsilon$, being $L \gg \varepsilon$ the infrared regulator introduced in the beginning of section 6. We remark that (F.55) is a special case of the general expression (1.4) (see (6.9) for the holographic case). Since at $\chi=\pi / 2$ we have that $\rho=\tilde{r}=L_{0} e^{\tau / L_{0}}$, in terms of this coordinate $\tau$ one finds that (F.55) becomes

$$
S_{A}=b L_{0} \frac{e^{\tau_{+} / L_{0}}-e^{\tau_{-} / L_{0}}}{\varepsilon}-f_{\alpha}(\gamma) \frac{\tau_{+}-\tau_{-}}{L_{0}}+O(1)
$$




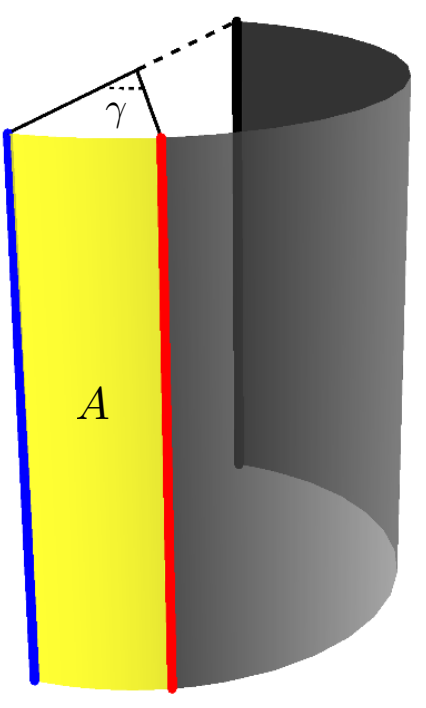

Figure 28. Part of the surface of a half cylinder, introduced in the appendix F.5 (see $d \tilde{s}^{2}$ in (F.54) for $\chi=\pi / 2$ ). This surface corresponds to the conformal boundary of the gravitational spacetimes depicted in figure 29. The yellow region is an infinite strip $A$ adjacent to the boundary.

where we used that $\rho_{\max }=L=L_{0} e^{\tau_{+} / L_{0}}$ and $\rho_{\min }=\varepsilon=L_{0} e^{\tau_{-} / L_{0}}$. These relations and the condition $L / \varepsilon \gg 1$ imply that $\left(\tau_{+}-\tau_{-}\right) / L_{0} \gg 1$.

In order to relate (F.56) to the expansion of the entanglement entropy of the infinite strip adjacent to the boundary, we take $L_{0} \rightarrow+\infty$ and $\gamma \rightarrow 0^{+}$such that $\ell=L_{0} \gamma$ is kept constant. Notice that the width $L_{0}(\pi-\gamma)$ of the complementary region $B$ in the half cylinder of figure 28 diverges in this limit. Moreover, since $L_{0} \rightarrow+\infty$ we have that $L_{0}\left(e^{\tau_{+} / L_{0}}-e^{\tau_{-} / L_{0}}\right) \rightarrow \tau_{+}-\tau_{-}$in the r.h.s. of (F.56). Thus, in this regime (F.56) becomes

$$
S_{A}=b \frac{L_{\|}}{\varepsilon}+A_{0} L_{\|}+O(1) \quad \tau_{+}-\tau_{-}=L_{\|} \gg L_{0}
$$

where $O\left(\left(\tau_{+}^{2}-\tau_{-}^{2}\right) / L_{0}^{2}\right)$ term has been neglected and $A_{0}$ is defined as follows

$$
-\frac{f_{\alpha}(\gamma)}{L_{0}} \rightarrow A_{0} \quad \text { as } \quad\left\{\begin{array}{l}
L_{0} \rightarrow+\infty \\
\gamma \rightarrow 0^{+} \\
L_{0} \gamma=\ell
\end{array}\right.
$$

The expression (F.57) in a $\mathrm{BCFT}_{3}$ corresponds to the entanglement entropy of an infinite strip $\left(L_{\|} \gg \varepsilon\right)$ of width $\ell$ adjacent to the boundary.

The above discussion holds for any $\mathrm{BCFT}_{3}$ with a flat boundary. In the following we focus on the case of $\mathrm{AdS}_{4} / \mathrm{BCFT}_{3}$, where this relation between the infinite wedge and the infinite strip adjacent to the boundary can be explicitly checked.

In order to address the holographic case, let us consider a part of the Euclidean $\mathrm{AdS}_{4}$ spacetime in global coordinates, whose spacetime interval reads

$$
d s^{2}=\frac{d r^{2}}{1+r^{2} / L_{\mathrm{AdS}}^{2}}+\left(1+r^{2} / L_{\mathrm{AdS}}^{2}\right) d \tau^{2}+r^{2}\left(d \chi^{2}+(\sin \chi)^{2} d \phi^{2}\right)
$$



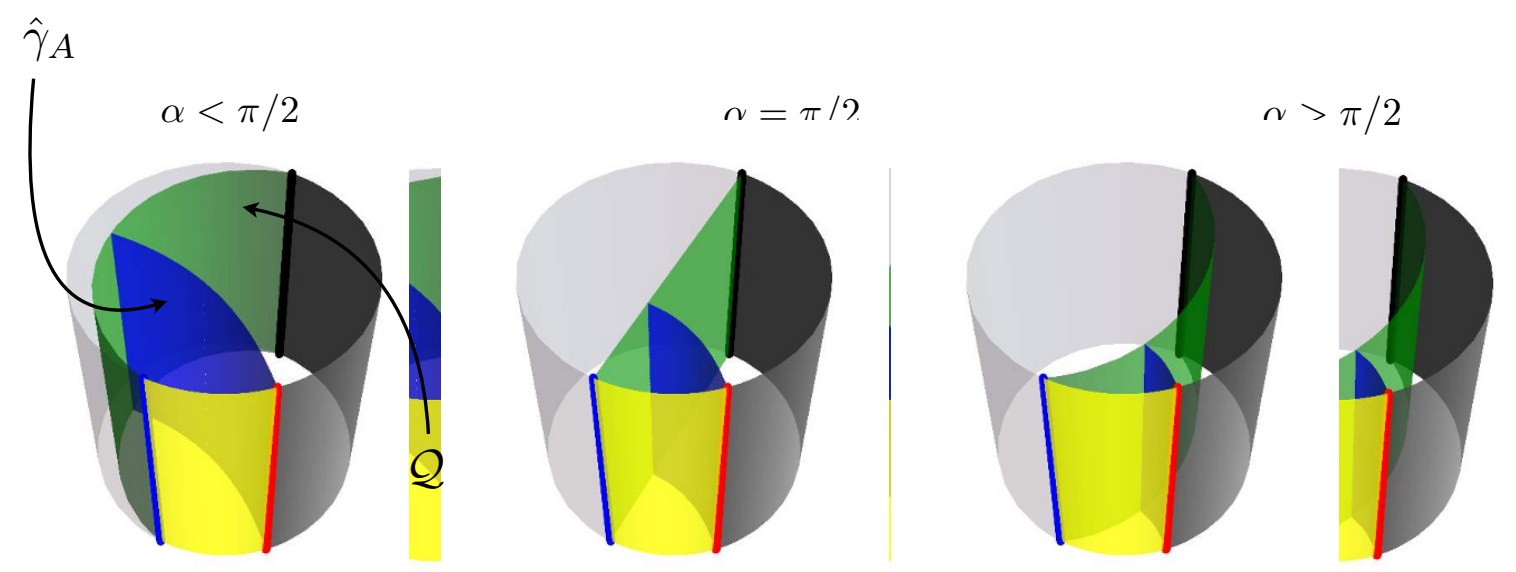

Figure 29. The spacetime (F.60), whose boundary is the union of the surface in figure 28 and of the green surface $\mathcal{Q}$ in (F.63), which depends on the parameter $\alpha \in(0, \pi)$. The blue surface is the minimal area surface $\hat{\gamma}_{A}$ corresponding to the infinite strip adjacent to the boundary (yellow region). The parameter $\alpha$ changes in the various panels: $\alpha=\pi / 10$ (left), $\alpha=\pi / 2$ (middle) and $\alpha=3 \pi / 4$ (right).

where $\tau \in \mathbb{R}, \chi \in[0, \pi], r \geqslant 0$ and $\phi \in[0,2 \pi)$, but the ranges of the last two coordinates are influenced by the occurrence of a constraint coming from (4.4). Indeed, we have that the conformal boundary corresponds to $r \rightarrow+\infty$ and $0 \leqslant \phi \leqslant \pi$. On the $\chi=\pi / 2$ slice, the induced metric is given by

$$
d s^{2}=\frac{d r^{2}}{1+r^{2} / L_{\mathrm{AdS}}^{2}}+\left(1+r^{2} / L_{\mathrm{AdS}}^{2}\right) d \tau^{2}+r^{2} d \phi^{2}
$$

By introducing the coordinates $(z, \rho)$ as follows

$$
r=L_{\mathrm{AdS}} \frac{\rho}{z} \quad \tanh \left(\tau / L_{\mathrm{AdS}}\right)=\frac{z^{2}+\rho^{2}-L_{\mathrm{AdS}}^{2}}{z^{2}+\rho^{2}+L_{\mathrm{AdS}}^{2}}
$$

one finds that (F.60) becomes

$$
d s^{2}=\frac{L_{\text {AdS }}^{2}}{z^{2}}\left(d z^{2}+d \rho^{2}+\rho^{2} d \phi^{2}\right)
$$

which is the metric of $\mathbb{H}_{3}$ (see (3.1)) in terms of the polar coordinates (F.1), whose conformal boundary corresponds to $z \rightarrow 0^{+}$.

From the definition (4.3) of half plane $\mathcal{Q}$ written in terms of the polar coordinates (F.1) and the first expression of (F.61), we find that the position of $\mathcal{Q}$ in the spacetime (F.60) is given by

$$
\mathcal{Q}: \quad r=-L_{\text {Ads }} \frac{\cot \alpha}{\sin \phi} \quad \begin{cases}\pi \leqslant \phi \leqslant 2 \pi & \alpha \in(0, \pi / 2) \\ 0 \leqslant \phi \leqslant \pi & \alpha \in(\pi / 2, \pi)\end{cases}
$$

In figure 29 the spacetime defined by (F.60) and constrained by (F.63) is the internal part of the cylinder enclosed by the green surface, which corresponds to $\mathcal{Q}$ and the darker half 
of the cylindrical surface, which is the conformal boundary of the spacetime (F.60) (see also figure 28). Since the conformal boundary is defined by $r \rightarrow+\infty$, in figure 29 the radial variable $\hat{r}=\left(2 L_{\mathrm{AdS}} / \pi\right) \arctan r$ has been employed. Notice that for $\alpha=\pi / 2$ half of the global $\mathrm{AdS}_{4}$ must be considered, as expected.

Close to this boundary the second expression of (F.61) becomes

$$
\tanh \left(\tau / L_{\mathrm{AdS}}\right)=\frac{\rho^{2}-L_{\mathrm{AdS}}^{2}}{\rho^{2}+L_{\mathrm{AdS}}^{2}} \quad \Longleftrightarrow \quad \rho=L_{\mathrm{AdS}} e^{\tau / L_{\mathrm{AdS}}}
$$

i.e. we recover the exponential change of coordinate reported in the text above (F.56), once the identification $L_{\mathrm{AdS}}=L_{0}$ is assumed.

In $\mathrm{AdS}_{4} / \mathrm{BCFT}_{3}$, by computing the holographic entanglement entropy of the infinite wedge adjacent to the boundary (section 6), we have found that (F.55) holds with $b=$ $L_{\text {AdS }}^{2} /\left(4 G_{\mathrm{N}}\right)$ and $f_{\alpha}(\gamma)=\frac{L_{\text {AdS }}^{2}}{4 G_{\mathrm{N}}} F_{\alpha}(\gamma)$, being $F_{\alpha}(\gamma)$ given by (6.5) and (6.10). By employing the results discussed in section 6.1 for the regime $\gamma \rightarrow 0^{+}$of $F_{\alpha}(\gamma)$ (namely (6.15) and the fact that $F_{\alpha}(\gamma) \rightarrow F_{\alpha}(0)$ when $\left.\alpha \leqslant \alpha_{c}\right)$ and the identification $L_{\mathrm{AdS}}=L_{0}$, we find that (F.58) in this case gives

$$
A_{0}=\frac{L_{\mathrm{AdS}}^{2}}{4 G_{\mathrm{N}}} \frac{a_{0}(\alpha)}{\ell}
$$

where $\ell=L_{\text {Ads }} \gamma$ and $a_{0}(\alpha)$ has been defined in (5.9). Plugging these results into (F.57), we recover the holographic entanglement entropy (5.9) of the infinite strip adjacent to the boundary, as expected.

As further consistency check of the relation between the infinite wedge and the infinite strip adjacent to the boundary, we find it worth considering the quantity $L_{\text {AdS }} \phi_{*}$ in the limit defined in (F.58) with $L_{0}=L_{\mathrm{AdS}}$. By employing (F.44) and the corresponding result for $\alpha \leqslant \alpha_{c}$, we find

$$
\begin{cases}L_{\mathrm{AdS}} \phi_{*}=-\frac{\cos \alpha \sqrt{\csc \alpha}}{\mathfrak{g}(\alpha)} L_{\mathrm{AdS}} \gamma+\cdots=-\frac{\cos \alpha \sqrt{\csc \alpha}}{\mathfrak{g}(\alpha)} \ell+\ldots & \alpha>\alpha_{c} \\ L_{\mathrm{AdS}} \phi_{*} \rightarrow-\infty & \alpha \leqslant \alpha_{c}\end{cases}
$$

In section 5.2 we have found that $x_{*}=-z_{*} \cot \alpha$ when $\alpha>\alpha_{c}$, with $z_{*}$ given by (5.3), while $x_{*} \rightarrow-\infty$ when $\alpha \leqslant \alpha_{c}$. Comparing these results with (F.66), we have that $x_{*}=L_{\text {Ads }} \phi_{*}$ in the limit that we are considering. This identification allows to interpret the transition between $\hat{\gamma}_{A}^{\text {con }}$ and $\hat{\gamma}_{A}^{\text {dis }}$ at $\alpha=\alpha_{c}$ for the infinite strip adjacent to the boundary (see section 5.2) in terms of the behavior of $\phi_{*}$ for $\gamma \rightarrow 0$. Indeed, when $\alpha>\alpha_{c}$, from (F.44) we have $\phi_{*} \rightarrow 0$ as $\gamma \rightarrow 0$, therefore $x_{*}$ remains finite and the minimal surface for the infinite strip is $\hat{\gamma}_{A}^{\text {con }}$. Instead, when $\alpha \leqslant \alpha_{c}$ the angle $\phi_{*}$ remains finite and negative, as discussed below (F.44). This means that $x_{*} \rightarrow-\infty$ for large $L_{\text {AdS }}$, which tells us that the minimal area surface for the infinite strip adjacent to the boundary is the vertical half plane $\hat{\gamma}_{A}^{\text {dis }}$.

\section{G The coefficient $A_{T}$ from holography}

In this appendix we describe the details of the holographic computation of the coefficient $A_{T}$ defined in (6.20) in the $\mathrm{AdS}_{d+2} / \mathrm{BCFT}_{d+1}$ setup of [63]. The main result of our analysis 
is the analytic expression of $A_{T}$ for arbitrary $d>1$. The special case of $d=2$ has been reported in section 6.2 .

The $\mathrm{AdS}_{d+2} / \mathrm{BCFT}_{d+1}$ construction of [63] has been described by employing the following metric

$$
d s^{2}=d \xi^{2}+\left[\cosh \left(\xi / L_{\mathrm{AdS}}\right)\right]^{2}\left(L_{\mathrm{AdS}}^{2} \frac{-d t^{2}+d \zeta^{2}+d \vec{y}^{2}}{\zeta^{2}}\right) \quad \zeta>0
$$

where $d \vec{y}^{2}$ is the Euclidean flat metric of $\mathbb{R}^{d-1}$. If $\xi \in \mathbb{R}$, then the metric (G.1) describes $\mathrm{AdS}_{d+2}$. Indeed, the change of coordinates

$$
z=\frac{\zeta}{\cosh \left(\xi / L_{\mathrm{AdS}}\right)} \quad x=-\zeta \tanh \left(\xi / L_{\mathrm{AdS}}\right)
$$

brings the metric (G.1) into the usual form (4.2) in terms of the Poincaré coordinates. Notice that on a generic $\xi=$ const slice of (G.1) the induced metric is the Poincaré metric of $\operatorname{AdS}_{d+1}$. In terms of the coordinates occurring in (G.1), the half hyperplane $\mathcal{Q}$ corresponds to a particular $\xi=$ const slice. From (G.2), we have that the conformal boundary where the $\mathrm{BCFT}_{d+1}$ is defined is defined by taking $\xi \rightarrow-\infty$ and $\zeta \rightarrow 0^{+}$, keeping the product $\zeta \xi$ fixed.

In order to make contact with the coordinates mainly employed throughout this manuscript, we find it convenient to introduce the angular coordinate $\psi \in(0, \pi)$ as follows

$$
\cot \psi=-\sinh \left(\xi / L_{\mathrm{AdS}}\right)
$$

From (G.2), it is straightforward to observe that

$$
\frac{z}{x}=-\frac{1}{\sinh \left(\xi / L_{\mathrm{AdS}}\right)}=\tan \psi
$$

In terms of the angular coordinate $\psi \in(0, \pi)$ defined in (G.3), the metric (G.1) becomes

$$
d s^{2}=\frac{L_{\mathrm{AdS}}^{2}}{(\sin \psi)^{2}}\left(d \psi^{2}+\frac{-d t^{2}+d \zeta^{2}+d \vec{y}^{2}}{\zeta^{2}}\right) \quad \zeta>0
$$

By employing the metric (G.5) in the $\mathrm{AdS}_{d+2} / \mathrm{BCFT}_{d+1}$ setup described in section 4, where the boundary of the $\mathrm{BCFT}_{d+1}$ is a flat hyperplane, we have that the half hyperplane $\mathcal{Q}$ in (4.3) is given by $\psi=\pi-\alpha$, with $\alpha \in(0, \pi)$, and the spacetime of the $\mathrm{BCFT}_{d+1}$ corresponds to the limit $\psi \rightarrow 0^{+}$. Indeed, (G.4) tells us that the limit $z \rightarrow 0^{+}$for fixed $x>0$ corresponds to $\psi \rightarrow 0^{+}$.

In order to find $A_{T}$ for the $\mathrm{AdS}_{d+2} / \mathrm{BCFT}_{d+1}$ construction proposed in [63], one introduces a non vanishing extrinsic curvature $k_{i j}$ for the boundary of the $\mathrm{BCFT}_{d+1}$ and solves the Einstein equations with the Neumann boundary condition $K_{i j}=(K-T) h_{i j}$ proposed by [63] perturbatively in $k_{i j}$, considering only the first order in the perturbation.

Since we consider the first non trivial order in the curvature of the boundary, the metric of the $\mathrm{BCFT}_{d+1}$ close to the boundary can be written as the follows

$$
d s^{2}=d x^{2}+\left(\eta_{i j}-2 x k_{i j}+\ldots\right) d Y^{i} d Y^{j}
$$


where $Y^{i}=(t, \vec{y})$ and $\eta_{i j}$ is the $d$ dimensional Minkowski metric. The dots denote higher order terms in the extrinsic curvature and in the distance $x$. In the literature this gauge choice is sometimes called geodesic slicing.

In order to find the bulk metric corresponding to (G.6), in the following we employ the ansatz recently suggested in [98] written in the coordinates adopted in (G.5). Also in [65] a similar analysis has been performed. In particular, let us consider the perturbation of (G.5) given by

$$
d s^{2}=\frac{L_{\mathrm{AdS}}^{2}}{(\sin \psi)^{2}}\left(d \psi^{2}+\frac{d \zeta^{2}+\left(\eta_{i j}-2 \zeta \cos \psi p_{d}(\psi) \kappa_{i j}\right) d Y^{i} d Y^{j}}{\zeta^{2}}\right)+O\left(k^{2}\right)
$$

where $\kappa_{i j}=k_{i j}-(k / d) \eta_{i j}$ is the traceless part of the extrinsic curvature and the boundary condition $p_{d}(0)=1$ is imposed to recover (G.6) for the $\mathrm{BCFT}_{d+1}$.

The metric (G.7) is a solution of the Einstein equations with negative cosmological constant up to $O\left(k^{2}\right)$ terms when $p_{d}(\theta)$ solves the following ordinary differential equation

$$
\sin (2 \psi) p_{d}^{\prime \prime}(\psi)-2\left[(d-2)(\cos \psi)^{2}+2\right] p_{d}^{\prime}(\psi)=0
$$

We remark that in (G.7) $\kappa_{i j}$ occurs in the perturbation (and not $k_{i j}$ ) without loss of generality. Indeed, if we start with a metric like (G.7) where $\kappa_{i j}$ is replaced by $k_{i j}$ and $\eta_{i j}$ by $\eta_{i j}\left[1+\zeta \cos \psi q_{d}(\psi) k\right]$, being $k$ the trace of $k_{i j}$, we find that the Einstein equations at the first perturbative order in the extrinsic curvature provide again the equation (G.8) for $p_{d}(\psi)$ besides another equation for the function $q_{d}(\psi)$. Otherwise, if we start with an ansatz like (G.7) with $\kappa_{i j}$ just replaced by $k_{i j}$, the Einstein equations to this order lead to (G.8), as expected, and also the condition that $k=0$.

The general solution of (G.8) reads

$$
p_{d}(\psi)=B_{d}+\frac{C_{d}}{\cos \psi}{ }_{2} F_{1}\left(-1 / 2,(1-d) / 2 ; 1 / 2 ;(\cos \psi)^{2}\right)
$$

where $B_{d}$ and $C_{d}$ are integration constants. The requirement that (G.9) satisfies the boundary condition $p_{d}(0)=1$ leads to

$$
B_{d}=1-\frac{\sqrt{\pi} \Gamma\left(\frac{d+1}{2}\right)}{\Gamma\left(\frac{d}{2}\right)} C_{d}
$$

Thus, the solution of (G.8) fulfilling the constraint $p_{d}(0)=1$ can be written as

$$
p_{d}(\psi)=1+C_{d} \mathcal{P}_{d}(\psi)
$$

where

$$
\mathcal{P}_{d}(\psi) \equiv \frac{1}{\cos \psi}{ }_{2} F_{1}\left(-1 / 2,(1-d) / 2 ; 1 / 2 ;(\cos \psi)^{2}\right)-\frac{\sqrt{\pi} \Gamma\left(\frac{d+1}{2}\right)}{\Gamma\left(\frac{d}{2}\right)}
$$

We find important to remark that the combination $p_{d}(\psi) \cos \psi$ occurring in the metric is smooth for $\psi \in(0, \pi)$. 
In the following we show that the constant $C_{d}$ in (G.11) can be fixed in order to have that the half hyperplane $\mathcal{Q}$ given by $\psi=\pi-\alpha$ is a solution of the Neumann boundary conditions $K_{a b}=(K-T) h_{a b}$ of [63] up to $O\left(k^{2}\right)$ terms.

Considering the metric $d s^{2}=g_{\mu \nu} d x^{\mu} d x^{\nu}$ defined in (G.7), the outward unit normal vector of the half hyperplane $\mathcal{Q}$ is $n^{\mu}=L_{\text {AdS }}^{-1}(\sin \alpha, \overrightarrow{0})$. As for the extrinsic curvature of $\mathcal{Q}$, we find that its non vanishing components are given by

$$
\begin{aligned}
K_{\zeta \zeta} & =\left.\frac{L_{\mathrm{AdS}} \sin \psi}{2 \zeta^{2}} \partial_{\psi}\left(\frac{1}{\sin ^{2} \psi}\right)\right|_{\psi=\pi-\alpha} \\
K_{Y^{i} Y^{j}} & =\left.\frac{L_{\mathrm{AdS}} \sin \psi}{2} \partial_{\psi}\left(\frac{1}{\zeta^{2} \sin ^{2} \psi} \delta_{i j}-\frac{2 p_{d}(\psi) \cos \psi}{\zeta \sin ^{2} \psi} k_{i j}\right)\right|_{\psi=\pi-\alpha}
\end{aligned}
$$

Taking the trace of the Neumann boundary conditions, it is straightforward to observe that they can be written as $K_{a b}=(T / d) h_{a b}$. Since $T=\left(d / L_{\text {AdS }}\right) \cos \alpha$ for the half hyperplane $\mathcal{Q}$, the condition to impose in order to get the solution given by $\mathcal{Q}$ becomes $K_{a b}=\left(\cos \alpha / L_{\mathrm{AdS}}\right) h_{a b}$ at $\psi=\pi-\alpha$. At $O(k)$, the component having $(a, b)=(\zeta, \zeta)$ is identically satisfied, while the components with $(a, b)=\left(Y^{i}, Y^{j}\right)$ lead to the following equation

$$
(\cot \alpha) p_{d}^{\prime}(\pi-\alpha)+p_{d}(\pi-\alpha)=0
$$

Plugging (G.11) into (G.15), we obtain an equation for the integration constant $C_{d}$ which can be easily solved. For $\alpha \in(0, \pi)$, we find

$$
\begin{aligned}
\frac{1}{C_{d}} & =-\mathcal{P}_{d}(\pi-\alpha)-\left.\cot \alpha \partial_{\psi} \mathcal{P}_{d}(\psi)\right|_{\psi=\pi-\alpha} \\
& =\frac{1}{\cos \alpha}{ }_{2} F_{1}\left(-1 / 2,(1-d) / 2 ; 1 / 2 ;(\cos \alpha)^{2}\right)-\frac{(\sin \alpha)^{d-1}}{\cos \alpha}+\frac{\sqrt{\pi} \Gamma\left(\frac{d+1}{2}\right)}{\Gamma\left(\frac{d}{2}\right)}
\end{aligned}
$$

Let us observe that $C_{d}=1 /(\pi-\alpha)^{d-1}+\ldots$ when $\alpha \rightarrow \pi$ and also that

$$
\partial_{\alpha}\left(1 / C_{d}\right)=-(d-1)(\sin \alpha)^{d-2}
$$

Comparing (G.17) with (C.12) it is straightforward to observe that $\partial_{\alpha}\left(1 / C_{d}\right)=\partial_{\alpha} \mathfrak{g}_{1 / d}$. This observation suggests to perform a direct comparison between (G.16) and (C.11), which provides the following intriguing relation

$$
\frac{1}{C_{d}(\alpha)}=\mathfrak{g}_{1 / d}(\alpha)
$$

It would be interesting to explore whether this observation leads to some physical insights.

We find it worth considering the special cases of $d=2$ and $d=3$ explicitly. In $\mathrm{AdS}_{4} / \mathrm{BCFT}_{3}$, the expressions (G.12) and (G.16) give respectively

$$
\mathcal{P}_{2}(\psi)=\tan \psi-\psi
$$

and

$$
C_{2}=\frac{1}{\pi-\alpha}
$$


In the case of $\mathrm{AdS}_{5} / \mathrm{BCFT}_{4}$ we have that (G.12) simplifies to

$$
\mathcal{P}_{3}(\psi)=\cos \psi+\sec \psi-2
$$

and (G.16) leads to

$$
C_{3}=\frac{1}{2(1+\cos \alpha)}
$$

In the remaining part of this appendix, we show that the constant $C_{d}$ is proportional to the constant $A_{T}$ defined by (6.20).

According to the holographic prescription of [86], the expansion close to the boundary of the one point function $\left\langle T_{i j}\right\rangle$ of the stress tensor in the $\mathrm{BCFT}_{3}$ is given by

$$
\left\langle T_{i j}\right\rangle=\frac{(d+1) L_{\mathrm{AdS}}^{d}}{16 \pi G_{\mathrm{N}}} \lim _{z \rightarrow 0} \frac{g_{i j}^{(1)}}{z^{d-1}} \quad x \rightarrow 0^{+}
$$

being $g_{i j}^{(1)}$ the $O(k)$ perturbation, which can be read from (G.7), finding

$$
g_{i j}^{(1)}=-\frac{2 \cos \psi p_{d}(\psi)}{(\sin \psi)^{2} \zeta} \kappa_{i j}
$$

where $p_{d}(\psi)$ is (G.11) with the constant $C_{d}$ given by (G.16)

In order to recover the expression (6.20) from (G.23), we have to exploit the relations among the various coordinates. In particular, from (G.3) we have that $\xi \rightarrow-\infty$ as $\psi \rightarrow 0^{+}$. Furthermore, taking this limit in the second expression in (G.2), one finds $\zeta \rightarrow x$. By considering the $O\left(\psi^{d+1}\right)$ term in the expansion of $p_{d}(\psi)$ for $\psi \rightarrow 0$, we obtain

$$
\lim _{z \rightarrow 0} \frac{g_{i j}^{(1)}}{z^{d-1}}=-\frac{2 C_{d}}{(d+1) x^{d}} \kappa_{i j}
$$

where we used that $z / x=\psi$ and $\zeta=x$ when $\psi \rightarrow 0^{+}$.

Finally, by plugging (G.25) into (G.23), we find that

$$
\left\langle T_{i j}\right\rangle=\frac{A_{T}}{x^{d}} \kappa_{i j}+\ldots \quad x \rightarrow 0^{+} \quad A_{T}=-\frac{L_{\mathrm{AdS}}^{d}}{8 \pi G_{\mathrm{N}}} C_{d}
$$

which corresponds to the expected $\mathrm{BCFT}_{d+1}$ behaviour (6.20). The proportionality relation between $A_{T}$ and the integration constant $C_{d}$ comes from the dual gravitational description of the $\mathrm{BCFT}_{d+1}$ at strong coupling.

We can write $A_{T}$ explicitly by employing the expression of $C_{d}$ that can be read from (G.16). The result is

$$
A_{T}=-\frac{L_{\mathrm{AdS}}^{d}}{8 \pi G_{\mathrm{N}}}\left[\frac{1}{\cos \alpha}{ }_{2} F_{1}\left(-1 / 2,(1-d) / 2 ; 1 / 2 ;(\cos \alpha)^{2}\right)-\frac{(\sin \alpha)^{d-1}}{\cos \alpha}+\frac{\sqrt{\pi} \Gamma\left(\frac{d+1}{2}\right)}{\Gamma\left(\frac{d}{2}\right)}\right]^{-1}
$$

We find worth remarking that $A_{T}$ can be written also in terms of the function $\mathfrak{g}_{d}(\alpha)$ defined in (C.11). From (G.26) and the relation (G.18), we obtain

$$
A_{T}=-\frac{L_{\mathrm{AdS}}^{d}}{8 \pi G_{\mathrm{N}}} \frac{1}{\mathfrak{g}_{1 / d}(\alpha)}
$$


The function $A_{T}(\alpha)$ is negative and decreasing function in the range $\alpha \in(0, \pi)$, Indeed, for $\alpha=0$ we find

$$
\left.A_{T}\right|_{\alpha=0}=\frac{L_{\mathrm{AdS}}^{d}}{8 \pi G_{\mathrm{N}}}\left(\frac{2 \sqrt{\pi} \Gamma\left(\frac{d+1}{2}\right)}{\Gamma\left(\frac{d}{2}\right)}-\delta_{d, 1}\right)^{-1}
$$

which is negative for every value of $d$. Moreover, from (G.17) it is straightforward to observe that

$$
\partial_{\alpha} A_{T}(\alpha)=\frac{L_{\mathrm{AdS}}^{d}}{8 \pi G_{\mathrm{N}}} C_{d}^{2} \partial_{\alpha}\left(1 / C_{d}\right)=-\frac{L_{\mathrm{AdS}}^{d}}{8 \pi G_{\mathrm{N}}}(d-1)(\sin \alpha)^{d-2} C_{d}^{2}
$$

which implies $\partial_{\alpha} A_{T}(\alpha) \leqslant 0$ for $\alpha \in(0, \pi)$. Furthermore, let us notice that the behaviour of $C_{d}$ as $\alpha \rightarrow \pi$ leads to conclude that $A_{T}(\alpha)=-\frac{L_{\mathrm{AdS}}^{d}}{8 \pi G_{\mathrm{N}}}(\pi-\alpha)^{-(d-1)}$ in this limit.

In the special case of $d=2$, the expression (G.27) of $A_{T}$ simplifies to (6.23) and this result is crucial to observe the relation (6.24), which holds for $\alpha \in(0, \pi)$.

The computation described above has been recently done for $d=2$ and $d=3$ also in [98] and non smooth expressions for $A_{T}$ have been found in the regime $\alpha \in(0, \pi)$.

\section{$\mathrm{H}$ Check of the constraints for the corner functions}

In this appendix we check that the holographic corner functions derived in section 6 and section 7 for $\mathrm{AdS}_{4} / \mathrm{BCFT}_{3}$ satisfy the constraints found in section 2.

The corner function (7.1) fulfils the inequality (2.6) in a trivial way. Indeed, whenever the maximisation procedure selects $\widetilde{F}(\omega)$ (namely for either $\alpha \leqslant \alpha_{c}$ or $\omega \leqslant \omega_{c}$ when $\left.\alpha>\alpha_{c}\right)$, this constraints simply tells us that the corner function $\widetilde{F}(\omega)$ is convex. The property $\tilde{f}^{\prime \prime}(\theta) \geqslant 0$ for the generic corner function $\tilde{f}(\theta)$ has been derived from the strong subadditivity in [30] and, in the special case of the holographic corner function $\widetilde{F}(\omega)$ found in [27], the convexity is evident from its plot (see the solid curve in figure 4 ). When the second function in the r.h.s. of (7.1) is selected, the inequality (2.6) is saturated, as one can straightforwardly observe by using that $\tilde{\gamma}=\pi-(\omega+\gamma)$.

As for the constraint obtained from the configuration shown in the middle panel of figure 2, we find it worth specialising the inequality (2.7) to the holographic corner functions. By employing (7.1), we find

$$
\begin{aligned}
& F_{\alpha}\left(\omega_{1}+\omega_{2}+\gamma\right)-F_{\alpha}\left(\omega_{1}+\gamma\right) \geqslant \\
& \quad \geqslant \max \left\{\widetilde{F}\left(\omega_{1}+\omega_{2}\right), F_{\alpha}(\gamma)+F_{\alpha}\left(\omega_{1}+\omega_{2}+\gamma\right)\right\}-\max \left\{\widetilde{F}\left(\omega_{1}\right), F_{\alpha}(\gamma)+F_{\alpha}\left(\omega_{1}+\gamma\right)\right\}
\end{aligned}
$$

For the configurations such that in both the maximisations occurring in the r.h.s. of (H.1) the second function is selected, $F_{\alpha}(\gamma)$ simplifies in the r.h.s. and this inequality becomes a trivial identity. As for other configurations, the inequality (H.1) is a non trivial inequality. We checked numerically for some cases that it is verified but, unfortunately, we do not have a general proof.

The last constraint to check is (2.10). Specifying this inequality for the holographic corner function (7.1), we obtain

$$
F_{\alpha}(\gamma+\omega)+F_{\alpha}(\gamma) \leqslant \max \left\{\widetilde{F}(\omega), F_{\alpha}(\gamma)+F_{\alpha}(\omega+\gamma)\right\}
$$

It is straightforward to observe that this inequality is trivially true. 
Open Access. This article is distributed under the terms of the Creative Commons Attribution License (CC-BY 4.0), which permits any use, distribution and reproduction in any medium, provided the original author(s) and source are credited.

\section{References}

[1] L. Amico, R. Fazio, A. Osterloh and V. Vedral, Entanglement in many-body systems, Rev. Mod. Phys. 80 (2008) 517 [quant-ph/0703044] [INSPIRE].

[2] J. Eisert, M. Cramer and M.B. Plenio, Area laws for the entanglement entropy - a review, Rev. Mod. Phys. 82 (2010) 277 [arXiv:0808.3773] [InSPIRE].

[3] P. Calabrese, J. Cardy and B. Doyon eds., Entanglement entropy in extended quantum systems, J. Phys. A 42 (2009) 500301.

[4] M. Rangamani and T. Takayanagi, Holographic entanglement entropy, Lect. Notes Phys. 931 (2017) pp.1-246 [arXiv:1609.01287] [INSPIRE].

[5] S. Ryu and T. Takayanagi, Holographic derivation of entanglement entropy from AdS/CFT, Phys. Rev. Lett. 96 (2006) 181602 [hep-th/0603001] [INSPIRE].

[6] S. Ryu and T. Takayanagi, Aspects of holographic entanglement entropy, JHEP 08 (2006) 045 [hep-th/0605073] [INSPIRE].

[7] V.E. Hubeny, M. Rangamani and T. Takayanagi, A covariant holographic entanglement entropy proposal, JHEP 07 (2007) 062 [arXiv:0705.0016] [INSPIRE].

[8] M. Freedman and M. Headrick, Bit threads and holographic entanglement, Commun. Math. Phys. 352 (2017) 407 [arXiv: 1604.00354] [INSPIRE].

[9] C.G. Callan Jr. and F. Wilczek, On geometric entropy, Phys. Lett. B 333 (1994) 55 [hep-th/9401072] [INSPIRE].

[10] C. Holzhey, F. Larsen and F. Wilczek, Geometric and renormalized entropy in conformal field theory, Nucl. Phys. B 424 (1994) 443 [hep-th/9403108] [InSPIRE].

[11] G. Vidal, J.I. Latorre, E. Rico and A. Kitaev, Entanglement in quantum critical phenomena, Phys. Rev. Lett. 90 (2003) 227902 [quant-ph/0211074] [INSPIRE].

[12] P. Calabrese and J.L. Cardy, Entanglement entropy and quantum field theory, J. Stat. Mech. 06 (2004) P06002 [hep-th/0405152] [INSPIRE].

[13] P. Calabrese, J. Cardy and E. Tonni, Entanglement entropy of two disjoint intervals in conformal field theory, J. Stat. Mech. 11 (2009) P11001 [arXiv:0905.2069] [INSPIRE].

[14] M. Headrick, Entanglement Rényi entropies in holographic theories, Phys. Rev. D 82 (2010) 126010 [arXiv:1006.0047] [INSPIRE].

[15] P. Calabrese, J. Cardy and E. Tonni, Entanglement entropy of two disjoint intervals in conformal field theory II, J. Stat. Mech. 01 (2011) P01021 [arXiv:1011.5482] [INSPIRE].

[16] A. Coser, L. Tagliacozzo and E. Tonni, On Rényi entropies of disjoint intervals in conformal field theory, J. Stat. Mech. 01 (2014) P01008 [arXiv:1309.2189] [InSPIRE].

[17] R.D. Sorkin, 1983 paper on entanglement entropy: "on the entropy of the vacuum outside a horizon", in Tenth international conference on general relativity and gravitation (held Padova Italy, 4-9 July 1983), contributed papers, vol. II, (1983), pg. 734 [arXiv: 1402.3589] [INSPIRE].

[18] L. Bombelli, R.K. Koul, J. Lee and R.D. Sorkin, A quantum source of entropy for black holes, Phys. Rev. D 34 (1986) 373 [InSPIRE]. 
[19] M. Srednicki, Entropy and area, Phys. Rev. Lett. 71 (1993) 666 [hep-th/9303048] [INSPIRE].

[20] R.C. Myers and A. Sinha, Seeing a c-theorem with holography, Phys. Rev. D 82 (2010) 046006 [arXiv: 1006.1263] [INSPIRE].

[21] R.C. Myers and A. Sinha, Holographic c-theorems in arbitrary dimensions, JHEP 01 (2011) 125 [arXiv: 1011.5819] [INSPIRE].

[22] D.L. Jafferis, I.R. Klebanov, S.S. Pufu and B.R. Safdi, Towards the F-theorem: $N=2$ field theories on the three-sphere, JHEP 06 (2011) 102 [arXiv:1103.1181] [INSPIRE].

[23] I.R. Klebanov, S.S. Pufu and B.R. Safdi, F-theorem without supersymmetry, JHEP 10 (2011) 038 [arXiv: 1105.4598] [INSPIRE].

[24] H. Liu and M. Mezei, A refinement of entanglement entropy and the number of degrees of freedom, JHEP 04 (2013) 162 [arXiv: 1202.2070] [INSPIRE].

[25] H. Casini and M. Huerta, On the RG running of the entanglement entropy of a circle, Phys. Rev. D 85 (2012) 125016 [arXiv:1202.5650] [INSPIRE].

[26] J.M. Maldacena, Wilson loops in large-N field theories, Phys. Rev. Lett. 80 (1998) 4859 [hep-th/9803002] [INSPIRE].

[27] N. Drukker, D.J. Gross and H. Ooguri, Wilson loops and minimal surfaces, Phys. Rev. D 60 (1999) 125006 [hep-th/9904191] [INSPIRE].

[28] E. Fradkin and J.E. Moore, Entanglement entropy of $2 D$ conformal quantum critical points: hearing the shape of a quantum drum, Phys. Rev. Lett. 97 (2006) 050404 [cond-mat/0605683] [INSPIRE].

[29] H. Casini and M. Huerta, Universal terms for the entanglement entropy in $2+1$ dimensions, Nucl. Phys. B 764 (2007) 183 [hep-th/0606256] [INSPIRE].

[30] T. Hirata and T. Takayanagi, AdS/CFT and strong subadditivity of entanglement entropy, JHEP 02 (2007) 042 [hep-th/0608213] [INSPIRE].

[31] H. Casini, M. Huerta and L. Leitao, Entanglement entropy for a Dirac fermion in three dimensions: vertex contribution, Nucl. Phys. B 814 (2009) 594 [arXiv:0811.1968] [INSPIRE].

[32] S. Whitsitt, W. Witczak-Krempa and S. Sachdev, Entanglement entropy of the large- $N$ Wilson-Fisher conformal field theory, Phys. Rev. B 95 (2017) 045148 [arXiv:1610.06568] [INSPIRE].

[33] E.H. Lieb and M.B. Ruskai, Proof of the strong subadditivity of quantum-mechanical entropy, J. Math. Phys. 14 (1973) 1938 [InSPIRE].

[34] A. Kallin, K. Hyatt, R. Singh and R. Melko, Entanglement at a two-dimensional quantum critical point: a numerical linked-cluster expansion study, Phys. Rev. Lett. 110 (2013) 135702 [arXiv: 1212.5269].

[35] E.M. Stoudenmire, P. Gustainis, R. Johal, S. Wessel and R.G. Melko, Corner contribution to the entanglement entropy of strongly interacting $O(2)$ quantum critical systems in $2+1$ dimensions, Phys. Rev. B 90 (2014) 235106 [arXiv:1409.6327] [INSPIRE].

[36] A.B. Kallin, E.M. Stoudenmire, P. Fendley, R.R.P. Singh and R.G. Melko, Corner contribution to the entanglement entropy of an $O(3)$ quantum critical point in $2+1$ dimensions, J. Stat. Mech. 06 (2014) P06009 [arXiv:1401.3504] [InSPIRE].

[37] J.-M. Stéphan, S. Furukawa, G. Misguich and V. Pasquier, Shannon and entanglement entropies of one- and two-dimensional critical wave functions, Phys. Rev. B 80 (2009) 184421 [arXiv: 0906.1153]. 
[38] T. Devakul and R. Singh, Quantum critical universality and singular corner entanglement entropy of bilayer Heisenberg-Ising model, Phys. Rev. B 90 (2014) 064424 [arXiv: 1406.0185].

[39] J. Helmes, L.E. Hayward Sierens, A. Chandran, W. Witczak-Krempa and R.G. Melko, Universal corner entanglement of Dirac fermions and gapless bosons from the continuum to the lattice, Phys. Rev. B 94 (2016) 125142 [arXiv:1606.03096] [InSPIRE].

[40] P. Bueno, R.C. Myers and W. Witczak-Krempa, Universality of corner entanglement in conformal field theories, Phys. Rev. Lett. 115 (2015) 021602 [arXiv:1505. 04804] [INSPIRE].

[41] P. Bueno and R.C. Myers, Corner contributions to holographic entanglement entropy, JHEP 08 (2015) 068 [arXiv: 1505.07842] [INSPIRE].

[42] T. Faulkner, R.G. Leigh and O. Parrikar, Shape dependence of entanglement entropy in conformal field theories, JHEP 04 (2016) 088 [arXiv: 1511.05179] [INSPIRE].

[43] J.L. Cardy, Conformal invariance and surface critical behavior, Nucl. Phys. B 240 (1984) 514 [INSPIRE].

[44] J.L. Cardy, Boundary conditions, fusion rules and the Verlinde formula, Nucl. Phys. B 324 (1989) 581 [INSPIRE].

[45] P. Di Francesco, P. Mathieu and D. Senechal, Conformal field theory, Springer-Verlag, New York U.S.A., (1997) [INSPIRE].

[46] J.L. Cardy, Boundary conformal field theory, hep-th/0411189 [INSPIRE].

[47] D.M. McAvity and H. Osborn, Energy momentum tensor in conformal field theories near a boundary, Nucl. Phys. B 406 (1993) 655 [hep-th/9302068] [INSPIRE].

[48] D.M. McAvity and H. Osborn, Conformal field theories near a boundary in general dimensions, Nucl. Phys. B 455 (1995) 522 [cond-mat/9505127] [INSPIRE].

[49] K. Jensen and A. O'Bannon, Constraint on defect and boundary renormalization group flows, Phys. Rev. Lett. 116 (2016) 091601 [arXiv: 1509.02160] [INSPIRE].

[50] S.N. Solodukhin, Boundary terms of conformal anomaly, Phys. Lett. B 752 (2016) 131 [arXiv: 1510.04566] [INSPIRE].

[51] C.P. Herzog, K.-W. Huang and K. Jensen, Universal entanglement and boundary geometry in conformal field theory, JHEP 01 (2016) 162 [arXiv:1510.00021] [INSPIRE].

[52] D. Fursaev, Conformal anomalies of CFT's with boundaries, JHEP 12 (2015) 112 [arXiv: 1510.01427] [INSPIRE].

[53] K.-W. Huang, Boundary anomalies and correlation functions, JHEP 08 (2016) 013 [arXiv: 1604.02138] [INSPIRE].

[54] C.P. Herzog and K.-W. Huang, Boundary conformal field theory and a boundary central charge, JHEP 10 (2017) 189 [arXiv: 1707.06224] [INSPIRE].

[55] K. Jensen and A. O'Bannon, Holography, entanglement entropy and conformal field theories with boundaries or defects, Phys. Rev. D 88 (2013) 106006 [arXiv:1309.4523] [INSPIRE].

[56] D.V. Fursaev and S.N. Solodukhin, Anomalies, entropy and boundaries, Phys. Rev. D 93 (2016) 084021 [arXiv:1601.06418] [INSPIRE].

[57] C. Berthiere and S.N. Solodukhin, Boundary effects in entanglement entropy, Nucl. Phys. B 910 (2016) 823 [arXiv:1604.07571] [INSPIRE].

[58] M. Headrick and T. Takayanagi, A holographic proof of the strong subadditivity of entanglement entropy, Phys. Rev. D 76 (2007) 106013 [arXiv:0704.3719] [INSPIRE]. 
[59] R.C. Myers and A. Singh, Entanglement entropy for singular surfaces, JHEP 09 (2012) 013 [arXiv: 1206.5225] [INSPIRE].

[60] P. Fonda, L. Giomi, A. Salvio and E. Tonni, On shape dependence of holographic mutual information in $A d S_{4}, J H E P 02$ (2015) 005 [arXiv: 1411.3608] [INSPIRE].

[61] P. Fonda, D. Seminara and E. Tonni, On shape dependence of holographic entanglement entropy in $A d S_{4} / C F T_{3}, J H E P 12$ (2015) 037 [arXiv:1510.03664] [INSPIRE].

[62] M.R. Mohammadi Mozaffar, A. Mollabashi and F. Omidi, Holographic mutual information for singular surfaces, JHEP 12 (2015) 082 [arXiv: 1511.00244] [INSPIRE].

[63] T. Takayanagi, Holographic dual of BCFT, Phys. Rev. Lett. 107 (2011) 101602 [arXiv: 1105.5165] [INSPIRE].

[64] M. Fujita, T. Takayanagi and E. Tonni, Aspects of AdS/BCFT, JHEP 11 (2011) 043 [arXiv:1108.5152] [INSPIRE].

[65] M. Nozaki, T. Takayanagi and T. Ugajin, Central charges for BCFTs and holography, JHEP 06 (2012) 066 [arXiv:1205.1573] [InSPIRE].

[66] C. Bachas, Asymptotic symmetries of AdS $S_{2}$-branes, hep-th/0205115 [INSPIRE].

[67] A. Karch and L. Randall, Locally localized gravity, JHEP 05 (2001) 008 [hep-th/0011156] [INSPIRE].

[68] O. DeWolfe, D.Z. Freedman and H. Ooguri, Holography and defect conformal field theories, Phys. Rev. D 66 (2002) 025009 [hep-th/0111135] [inSPIRE].

[69] T. Azeyanagi, A. Karch, T. Takayanagi and E.G. Thompson, Holographic calculation of boundary entropy, JHEP 03 (2008) 054 [arXiv:0712.1850] [INSPIRE].

[70] J. Erdmenger, C. Hoyos, A. O'Bannon and J. Wu, A holographic model of the Kondo effect, JHEP 12 (2013) 086 [arXiv:1310.3271] [INSPIRE].

[71] J. Erdmenger, M. Flory, C. Hoyos, M.-N. Newrzella and J.M.S. Wu, Entanglement entropy in a holographic Kondo model, Fortsch. Phys. 64 (2016) 109 [arXiv:1511. 03666] [InSPIRE].

[72] R.-X. Miao, C.-S. Chu and W.-Z. Guo, New proposal for a holographic boundary conformal field theory, Phys. Rev. D 96 (2017) 046005 [arXiv: 1701.04275] [InSPIRE].

[73] C.-S. Chu, R.-X. Miao and W.-Z. Guo, On new proposal for holographic BCFT, JHEP 04 (2017) 089 [arXiv: 1701.07202] [INSPIRE].

[74] A. Faraji Astaneh and S.N. Solodukhin, Holographic calculation of boundary terms in conformal anomaly, Phys. Lett. B 769 (2017) 25 [arXiv: 1702.00566] [INSPIRE].

[75] A. Faraji Astaneh, C. Berthiere, D. Fursaev and S.N. Solodukhin, Holographic calculation of entanglement entropy in the presence of boundaries, Phys. Rev. D 95 (2017) 106013 [arXiv: 1703.04186] [INSPIRE].

[76] K. Brakke, The surface evolver, Experiment. Math. 1 (1992) 141.

[77] Surface Evolver program webpage, http://www.susqu.edu/brakke/evolver/evolver.html.

[78] G. Hayward, Gravitational action for space-times with nonsmooth boundaries, Phys. Rev. D 47 (1993) 3275 [INSPIRE].

[79] S.W. Hawking and C.J. Hunter, The gravitational Hamiltonian in the presence of nonorthogonal boundaries, Class. Quant. Grav. 13 (1996) 2735 [gr-qc/9603050] [INSPIRE].

[80] M. Henningson and K. Skenderis, The holographic Weyl anomaly, JHEP 07 (1998) 023 [hep-th/9806087] [INSPIRE]. 
[81] M. Bianchi, D.Z. Freedman and K. Skenderis, How to go with an RG flow, JHEP 08 (2001) 041 [hep-th/0105276] [INSPIRE].

[82] M. Bianchi, D.Z. Freedman and K. Skenderis, Holographic renormalization, Nucl. Phys. B 631 (2002) 159 [hep-th/0112119] [INSPIRE].

[83] K. Skenderis, Lecture notes on holographic renormalization, Class. Quant. Grav. 19 (2002) 5849 [hep-th/0209067] [INSPIRE].

[84] V. Balasubramanian and P. Kraus, A stress tensor for anti-de Sitter gravity, Commun. Math. Phys. 208 (1999) 413 [hep-th/9902121] [INSPIRE].

[85] R.C. Myers, Stress tensors and Casimir energies in the AdS/CFT correspondence, Phys. Rev. D 60 (1999) 046002 [hep-th/9903203] [INSPIRE].

[86] S. de Haro, S.N. Solodukhin and K. Skenderis, Holographic reconstruction of space-time and renormalization in the AdS/CFT correspondence, Commun. Math. Phys. 217 (2001) 595 [hep-th/0002230] [INSPIRE].

[87] H. Casini, M. Huerta and R.C. Myers, Towards a derivation of holographic entanglement entropy, JHEP 05 (2011) 036 [arXiv:1102.0440] [INSPIRE].

[88] J.K. Erickson, G.W. Semenoff and K. Zarembo, Wilson loops in $N=4$ supersymmetric Yang-Mills theory, Nucl. Phys. B 582 (2000) 155 [hep-th/0003055] [INSPIRE].

[89] K. Nagasaki, H. Tanida and S. Yamaguchi, Holographic interface-particle potential, JHEP 01 (2012) 139 [arXiv:1109.1927] [INSPIRE].

[90] E. Tonni, Holographic entanglement entropy: near horizon geometry and disconnected regions, JHEP 05 (2011) 004 [arXiv:1011.0166] [INSPIRE].

[91] E. Tonni, Corner contributions to holographic entanglement entropy in $A d S_{4} / B C F T_{3}$, talk delivered at Integrability in Gauge and String Theory (IGST 2017), École Normale Supérieure, Paris France, 20 July 2017.

[92] M. Preti, D. Trancanelli and E. Vescovi, Quark-antiquark potential in defect conformal field theory, JHEP 10 (2017) 079 [arXiv:1708.04884] [INSPIRE].

[93] N. Drukker and V. Forini, Generalized quark-antiquark potential at weak and strong coupling, JHEP 06 (2011) 131 [arXiv:1105.5144] [INSPIRE].

[94] D. Correa, J. Maldacena and A. Sever, The quark anti-quark potential and the cusp anomalous dimension from a TBA equation, JHEP 08 (2012) 134 [arXiv:1203.1913] [INSPIRE].

[95] H. Liu and A.A. Tseytlin, $D=4$ super Yang-Mills, $D=5$ gauged supergravity and $D=4$ conformal supergravity, Nucl. Phys. B 533 (1998) 88 [hep-th/9804083] [INSPIRE].

[96] A. Buchel, J. Escobedo, R.C. Myers, M.F. Paulos, A. Sinha and M. Smolkin, Holographic GB gravity in arbitrary dimensions, JHEP 03 (2010) 111 [arXiv:0911.4257] [InSPIRE].

[97] D. Deutsch and P. Candelas, Boundary effects in quantum field theory, Phys. Rev. D 20 (1979) 3063 [INSPIRE].

[98] R.-X. Miao and C.-S. Chu, Universality for shape dependence of Casimir effects from Weyl anomaly, arXiv:1706.09652 [INSPIRE].

[99] H. Bateman, Higher transcendental functions, volume I, McGraw-Hill, New York U.S.A., (1953).

[100] M. Abramowitz and I. Stegun, Handbook of mathematical functions, Dover Publications, New York U.S.A., (1972). 